Portland State University

PDXScholar

$5-1-1968$

\title{
Theoretical calculations of kinetic isotope effects
}

Paul Chien-Wei Chang

Portland State University

Follow this and additional works at: https://pdxscholar.library.pdx.edu/open_access_etds Let us know how access to this document benefits you.

\section{Recommended Citation}

Chang, Paul Chien-Wei, "Theoretical calculations of kinetic isotope effects" (1968). Dissertations and Theses. Paper 785.

https://doi.org/10.15760/etd.785

This Thesis is brought to you for free and open access. It has been accepted for inclusion in Dissertations and Theses by an authorized administrator of PDXScholar. Please contact us if we can make this document more accessible: pdxscholar@pdx.edu. 
Paul Chien-We1 Chang

(Name) for the $\frac{M . S}{\text { (Degree) }}$ in $\frac{\text { Chemistry }}{\text { (Major) }}$

Date thesis is presented

May 9,1968

Title Theoretical Calculations of Kinet1c Isotope Effects

Abstract approved

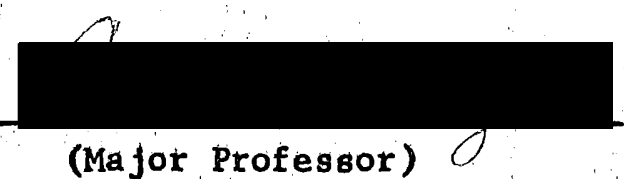

Treating the activated complex for unfmolecular dissoctation as uncoupled fragments simplifies the calculation of transition-state frequencies in the quasi-equilibrium-theory formulation of an isotopic rate-constant ratio. The general method is applied to the specific case of the intramolecular kinetic isotope effect in decarboxylation of malonic acid. The fragment models are further simplifled by means of the "cut-off" approximation of Sterm and Wolfsberg. These model calculations are in agreement with the presently avallable experimental results. Applicability to reactions involving simultaneous multiple bond rupture or formation is noted. 
THEORETICAL CALCULATIONS OF

KINETIC ISOTOPE EFFECTS

by

Paul Chlen-Wel Chang

THESIS

Presented to the Graduate Faculty

of Portland state College

In Partial Fulfiliment of Requirements

For the Degree of Master of Sclence

Department of Chemlotry

Under the Supervision of Dr. Carole R. Gatz

Portland, Oregon

May 9, 1968

PORTLAND STATE COLLEGE 
APPROVED:

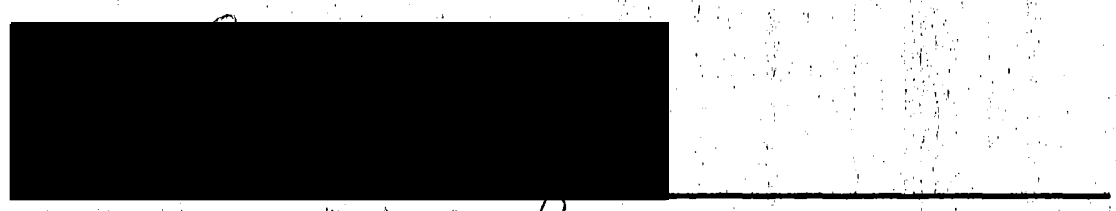

Professor of Chemlstry

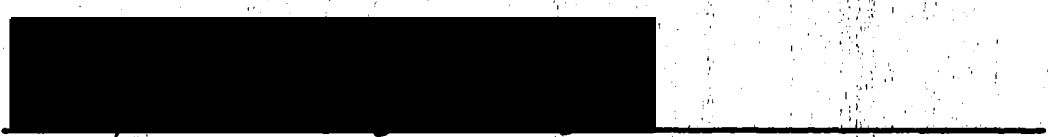

Head of Department of Chemistry

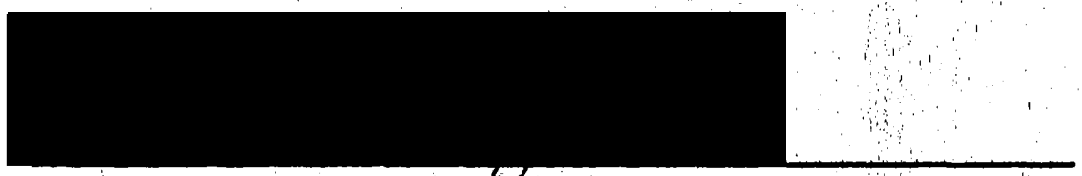

Dean of Graduate Schod

Date thesis presented May 9, 1968

Typed by C.H. 
The author 18 greatly Indebted to Dr. Carole R. Gatz for suggesting this research problem, providing encouragement and numerous helpful comments through all phases of the investigation.

It is Impossible for the author to thank everyone who has contributed to this research. To Dr. David Mcclure he owes a debt for the valuable suggestions with regard to the mathematical aspects of thls work. To Dr. Robert Broussard he owes a debt of making the computations possible. To many of his frlends, especially James Plenovi, goes the credit for the assistance in preparing the computer: programs. The author speclally wants to thank his wife, Jackle, with love for her infinlte patience and endless support.

This thesis is dedicated to my parents

$$
\begin{gathered}
\text { Wen-Yin, Chang } \\
\text { and }
\end{gathered}
$$$$
\text { Hoo-Lin Chang }
$$ 
CONTENTS

ACKNOWLEDGMENT

CHAPTER I - INTRODUCTION

I

1. General Discussion

2. Brief History of the Study of Isotope Effects

3. Statement and General Purpose of the Investigation

CHAPTER II - REACTION RATE THEORY

8

1. Arrhenius Equation

2. Potential Energy Surface (PES)

3. Quasi-Equilibrium Theory (QET)

4. Statistical Treatment

5. Absolute Rate Theory

6. Isotope Effects

CHAPTER III - MOLECULAR VIBRATIONS

1. Separation of Energy

2. Coordinate Systems of Small Vibrations

CHAPTER IV - FORMULATIONS AND CALCULATIONS 70

1. Molecular Fragmentation

2. The "G" Matrix

3. Calculations and Results 
CHAPTER $V$ - SUMMARY AND FURTHER CALCULATIONS

1. General Summary.

2. Further Calculations

APPENDIX - COMPUTER PROGRAMS

111

1. Main Program

2. "G" Matrix

3. Eigen Value Problem.

4. Isotope Kinetic Rate Ratio. 


$$
\text { Chapter I - Introduction }
$$

1. General Discussion 2

2. Brief History of the study of Isotope Effects 5

3. Statement and General Purpose of the Investigation 7 
Chapter I - Introduction

1. General Discussion

The explosively expanding system of human knowledge of "Nature", If time is a partition factor, is composed of two subsystems; "tlme-varlant" and "tlme-invarlant". In the "time-variant". system there lles the major domain of "Chemical Kinetics".

In nearly all areas of sclentiflc research, whenever a given system is brought under investlgation, analysis normally involves the lsolation and definition of whatever elements or subsystems appear to contribute in some essential way to the whole system. There is, in other words, a tendency toward fundamentality. An extension of this tendency might appear to suggest that only when some basic bullding block of matter is defined will there be a truly adequate understanding of natural phenomena. The fact is that if such a substance exists, it has thus far eluded the best effort at detection or definition. Moreover, it is only because of the measurable interaction of organized matter that we attempt to define the properties of matter at all.

Thus while we strive to explain the objects of our research in the most fundamental terms possible, it may well be that even at the prime level of fundamentality, we may still be forced to deal with matter in terms of the relationships among organized and particulate entitles. 
Indeed, attempts to explain the propertles of matter at this level tend, if anything, away from fundamentallty. Investigation of Interparticle relationships, historically, has contributed more to a general understanding of matter than has preoccupation with the Intrinsic propertles of the particles themselves.

Among those attempts to probe the behavior of particulate matter is the Investigation of the mechanism of chemical reaction. A better understanding of the reaction mechanlsm is one of the Immedlate goals which may lead to the understanding of the general princlples of reactivity. Through a study of the rate of production of a certain substance as a function of the concentrations of reactants, it is frequently possible to find out how many entities of each $k$ ind have to come together.: And Information about the energy threshhold which must be surmounted before any cluster of reactants may pass into the product,' is generally provided by the temperature coefficlent of the reaction rate.

It has been concluded that, even with sufficlent energy, not every molecule can pass through the energy barrler. Hence the conformation theory of the transition state enters here. In order to obtain a clear view of the structures of the molecules during the course of reaction, we demand a set of definitely defined functions of each Individual particle involved. Unfortunately, because of the lack of exactness of the functlons stated by Heisenberg!s 
uncertalnty princlplel and the incompleteness of our knowledge, we would be, in general, satlsfled if we had an approximate idea of the structure and conformation of the transition state.

Theoretical predictions thus play a vital role in the game. In order to set up such a system which would produce accurate enough results for a significant comparison with the experimental date, the physlcal parameters have to be known with a great accuracy. Because of the cancellation effects of the errors during the computing process, the ratio of kinetic rates is a much better method to examine the validity of the theoretical model than others. By varying the Internal or external conditions of certain reactions to study the influence on the relative kinetic rate ratio, lsotopic substitution has been suggested as an easier method to test the assumptions made for a great number of reactions. The superiority of the lsotope method lies mainly in the possiblifty of making the substitution within the very reacting center and with a minimum of change in parameters. 
2. Brlef History of the Study of Isotope Effects

In the year 1919 Lindemann Initiated the study of isotope effects with a calculation of the difference in vapor pressures of lead isotopes. 2 The real interest of the investigators was not attracted untll 1933 when Urey and Rittenberg published the result of their calculations of the equillibrium constants for the reactions 3

$$
\begin{aligned}
& \mathrm{H}_{3}+2 \mathrm{DCl}=\mathrm{D}_{2}+\mathrm{HCl} \\
& \mathrm{H}_{2}+2 \mathrm{DI}=\mathrm{D}_{3}+2 \mathrm{HI} .
\end{aligned}
$$

Thereafter, the investigations of equilibrium isotope exchange reactions was extended to many systems, mostly involving lsotopes of hydrogen, carbon, oxygen and nitrogen.

In 1947, H. C. Urey published "The Thermodynamic Properties of Isotopic Substances", 4 which contains vibrational data and partition function ratios for many of the simpler isotoplc molecules, and Bigeleisen and Mayer 5 obtained general expression for a ratio of partition functions for isotope fractionation in equilibrium exchange reactions. During the following two years Beeck ${ }^{6}$ discovered a large carbon lsotope fractionation in the dissociation of propane upon electron impact, and Bigeleisen? combined the absolute rate theory with his previous work. In later years Polany ${ }^{\theta}$, Weston ${ }^{9}$, and Shavitt 10 proceeded by arriving at the best potential-energy surfaces for the reactions, andimaking absolute calculations of the rates of the reactions that are to be compared. Although 
the results of their investigations have thrown light on the question of obtaining the best methods for arriving at potential-energy surfaces, the actual computation of potentlal energy surface ls Impossibly complex for all but the simplest systems. 
3. Statement and General Purpose of the Investigation

Recent theoretical work has centered upon a search for approximated computable models that, if the calculated lootope effects are in agreement with experimental data, may provide some valuable information about reaction mechanlsms. Different theoretical models have been Investigated, but the results are somewhat disappointing. In addition to the extreme compexity involved in actual computation, the crudest models have often given predictions at least as good as those of more refined calculations. It is hoped that in developing a certain general method for calculating the kinetlc isotope effects, the actual computation may be simplified and the accuracy may be Improved.

In order to examine the validity of the investlgated method, the decarboxylation of malonic acid is chosen to be a trial; since this particular reaction has already been thoroughly investigated. The calculated kinetlc Isotope effects will be compared with the presently avallable experimental data ${ }^{12}$ of this reaction in the vapor phase. 
Chapter II - Reaction Rate Theory

1. Arrhenlus Equation

2. Potentlal Energy Surface (PES)

3. Quas:1-Equillibrlum Theory. (QET)

4. Statistical Treatment

A. Electronic Partition Function

8. Translational Partition Function

c. Vibrational Partition Function

D. Rotational Partition function

E. Rate of Chemical Reaction

5. Absolute Rate Theory

6. I sotope Effects

A. I sotope Effects on PES

B. Application of Rate Theory to I sotope Effects 
Chapter II - Reaction Rate Theory

1. Arrhenius Equation

Qualitative observations of the rate of chemical reactions were recorded by early writers, but the first significant kinetic measurements may be sald to have been those of W/Ihelmy 13 , who in 1850 measured the rate of inversion of sucrose and investigated the influence of concentration upon the rate. He came to the conclusion:

$$
c=C_{0} \exp (-k T)
$$

where $c_{0}=$ initial concentration of sugar

$$
\begin{aligned}
& c=\text { concentration of sugar at time } t \\
& k=\text { rate constant. }
\end{aligned}
$$

Later workers have obtained the same result. The important paper of Guldberg and Waage 14 pointed out that the laws of chemical equilibrium can be derlved from the kinetic laws by assuming that at equilibrium the rates of forward and reverse reactions are the same.

In 1889 the Arrhemlus law $^{15}$ was formulated.

$$
k=A \exp \left(E_{a} / R T\right)
$$

where $k$ is the kinetic rate constant, $E_{a}$ is the molar activation energy of the reaction, $R(\mathrm{cal} / \mathrm{mole}$ deg.) is the gas constant, $T$ is the absolute temperature, and $A$ is called the pre-exponential factor or frequency factor. According to Arrhenlus, Eq. (II-2) indicated that molecules must acquire a certain critical energy, $E_{a}$, before they can react; the Boltzmann factor, exp ( $\left.E_{a} / R T\right)$, being 
the fraction of molecules that possess the necessary energy. From the standpolnt of the Arrhenius law, a complete understanding of the factors determining the rate constant of a reaction involves an understanding of the activation energy and the pre-exponential factor. Although the quantum-mechanical theorles of molecular structures have been formulated in detall, the evaluation of activation energy still remains as an unsolved problem. The problem of calculating frequency factors on the basis of fundamental principles has been attacked in two ways, namely, the kinetic theory of collisions and the statistical mechanics approach. The latter approach will be introduced In thls Chapter. 
2. Potential Energy Surface

The activation energles of chemical reactions are most convenlently consldered using the method of potent|al-energy surfaces. The electronlc potentlal energy for a system of $n$ atoms undergoing "adiabatic" reaction can be considered by means of the Born-Oppenhelmer, 16 approximation as a continuous and single-valued function of the nuclear motion. In general, the interaction between two $(n+1)$-dimensional systems is an $n$-dimenslonal vector space. The behavior of nuclel thus can be described by motion on the surface.

The three-dimensional diagram in Fig. II-I is employed to Introduce this concept briefly. The potentialenergy surface for the reaction would possess low valleys corresponding to the energy states of reactant and product, and the valleys would be separated by regions of higher potentlal energy. The famlly of parabolas in the $x-Z$ plane represents all the possible reactant paths. The minimum of the trajectory of the maximum of ail the reaction paths is defined as an activation point. The reacting system at this point is defined as "activated complex" or transition state.

It is easily seen from Fig. II-l that if a system at an activation polnt moves along the direction of the reaction path $(X-a x i s)$, the value of its image in the range of the function, potential energy, decreases. And any change 
FIg. II-I

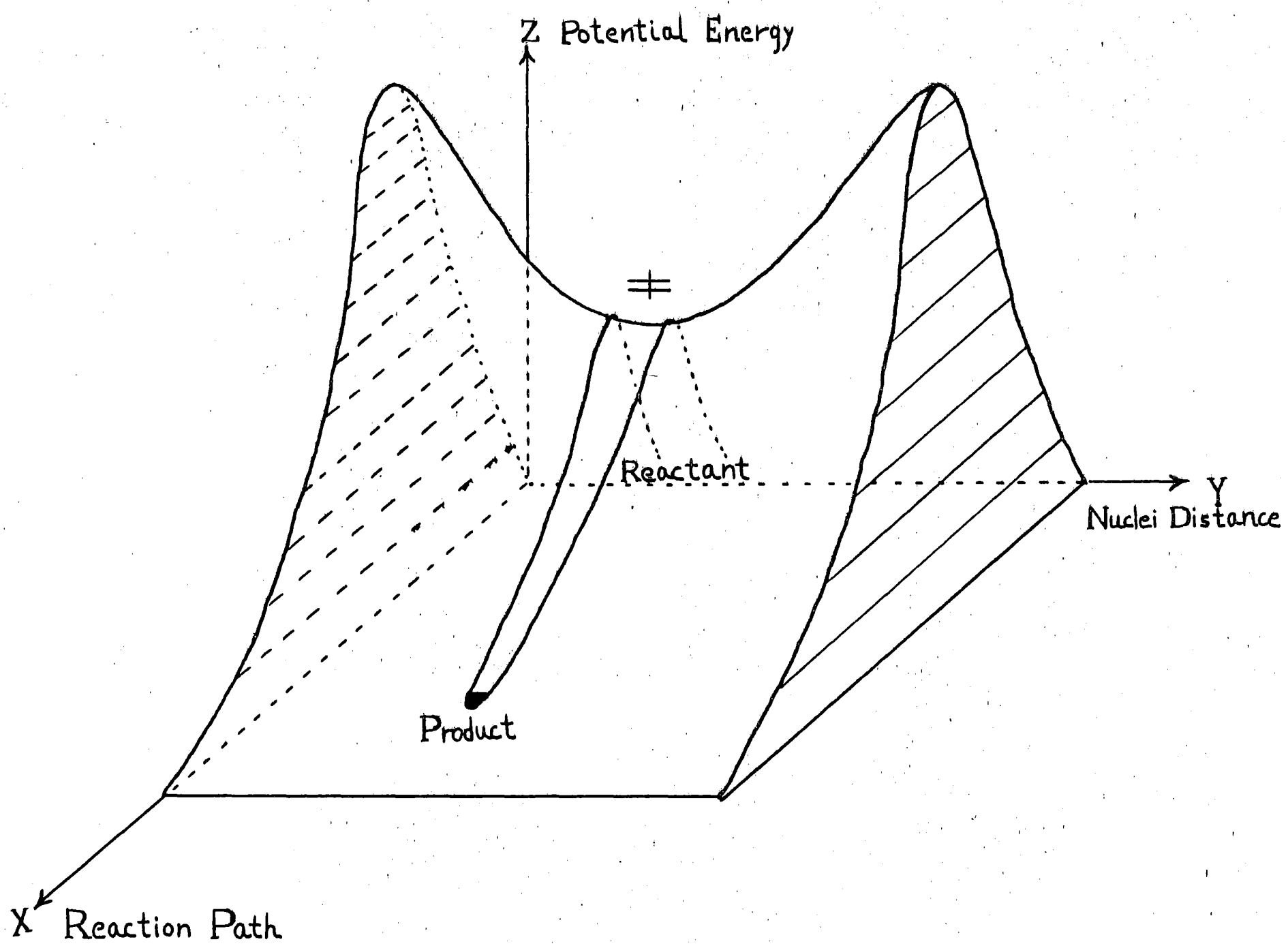


of value along the direction of the $Y$-axis would increase the potential energy. This speciflc member of the family is in general the reaction path, and the direction of steepest descent is defined as the reaction coordinate.

This type of potentlal-energy surface would provide detalled information about the reacting syotem in the transition-state. But, in transforming the real physical' system to the mathematical system, the actual computation is extremely complex for even the simplest systems. Hence the transition-state approach of the absolute rate theory has become a general method for calculating reaction rates from simple structural parameters by means of the principles of statistical mechanics. 
3. Quasi-Equilibrium Theory

During the course of chemical reactions it is important to distinguish clearly between two aspects of the problem: one is concerned with the direction and extent of chemical change, the other with the rate with which it takes place. These two systems, "time-invariant" and "time-variant", have no simple connections. A chemical reaction between two substances may occur almost to completion, but the time for even a very small fraction of the molecules to react may be extremely long. Although the theory of equilibria and the theory of kinetic rates are non-parametric, there is a close relationship between the two. This is so because the molecules undergoing reaction and passing through activated states which can be regarded as in equilibrium with the molecules in their normal states. The concentration of activated molecules can therefore be calculated by ordinary equilibrium theory, so that if the probability of the decomposition of activated molecules 18 known, the rate of reaction can be calculated. In general, this is called quasi-equilibrium theory.

The problem of calculating rates therefore resolves itself into two parts: the calculation of the concentrations of activated molecules and the calculation of the rates of reaction of the activated molecules. 
Consider a reaction

$$
A+B=X=C+D
$$

which has proceeded to equillbrium. Then, according to our assumption, the actlvated complex $x$ will also be in equilibrium with the reactants and products and their concentration may be calculated accurately by the methods of statistical mechanics in terms of the concentrations of $A$ and $B$.

The theory Involves the hypothesis, however, that even when the reactants and products are not at equilibrium with each other, the activated complexes are at equilibrium with the reactants. When we state that the activated complexes are in equilibrium with the reactants, we refer only to those complexes that in the Immediate past were reactant molecules.

It is to be emphasized that there is no assumption that there is a classical type of equilibrium between initial and activated states; addition to the system of activated complexes moving from the Initial to the final state would not disturb the equillibrlum, as would be required if the equilibrlum were classical.

The quasi-equillibrium theory. Is supported by the derlvations made by $B$ lshop and Laldier ${ }^{18}$. They concluded that reactions are satisfactorlily interpreted on the basis of the quasi-equilibrium assumption, provided that $E_{a} / R T$ for the reaction has a value of 5 or larger: If the value of $E_{a} / R T$ is smaller than 5 , the reaction will occur 
so rapidly that there can no longer be equillbrlum even among the reactant molecules; the more energetic species will be removed more rapidly than the supply of them can be replenished, and there will not be a Boltzmann distribution of reactant molecules. 
4. Statistical Treatment

The single molecule partition function $Q$ is defined as

$$
Q=\Sigma q_{1} \exp \left[-E_{1} / K T\right]
$$

where $K$ is the Boltzmann constant, $E_{\mathcal{F}}$ is the total energy

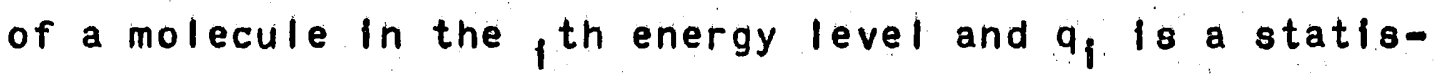
tical welght equal to the number of different molecular states that have the same energy, $E_{j}$. The individual products $q_{1} \exp \left[-E_{1} / K T\right]$ represent the relative probabillty of finding a molecule in the thenergy level, with energy $E_{1}$, and 80

$$
P\left(E_{1}\right)=q_{1} \exp \left[-E_{1} / K T\right]
$$

represents a discrete energy distribution function for molecules restricted to quantlzed energy states.

To a good approximation, the total energy of a molecule can be separated Into Independent terms,

$$
E_{t}=E_{e}+E_{v}+E_{r o t}+E_{t r}
$$

where $E_{t}$ is the total energy of a molecule, $E_{e}$ is the electronlc energy, $E_{v}$ is the vibrational energy arising from the internal motions of the nuclei, Erot is the rotational energy of the molecule as a whole, and $E_{t r}$ is the translational energy.

According to the Boltzmann formulation for the distribution function of a set of molecules among varlous energy states we have 


$$
P(E) \propto \exp [-E / K T]
$$

since $E$ is separable,

$$
P(E) \propto \exp \left[\left(-E_{e}-E_{v}-E_{r o t}-E_{t r}\right) / K T\right]
$$

and for the partition function we can write

$$
\begin{aligned}
Q= & {\left[\Sigma q_{e} \exp \left(-E_{e} / K T\right)\right]\left[\Sigma q_{v} \exp \left(-E_{v} / K T\right)\right] } \\
& \times\left[\Sigma q_{r o t} \exp \left(-E_{r o t} / K T\right)\right]\left[\Sigma q_{t r} \exp \left(-E_{t r} / K T\right)\right] \\
= & Q_{e} Q_{v} Q_{r o t}{ }^{Q} t r
\end{aligned}
$$

since the individual sums are independent. Thus for energies whlch are separable in thls manner we can say that the partition function $Q$ will be a product of Individual partition functions for the different kinds of energy.

\section{A. Electronic Partition Function}

The partition function for electronic energy is calculated directly from the observed electronic levels of the atom or molecule, using the relationship

$$
Q_{e}=\Sigma q_{e} \exp \left[-E_{e} / K T\right]
$$

At ordinary temperatures the excited electronic levels of an atom or molecule are usually too high to make a significant contribution to the partition function. If the lowest state is a singlet state, the statistical weight $q_{e}$ is unity, so that if the lowest state is taken as the zero level and all other levels are sufficlently high, the partition function is approximately unity. In general, it may be assumed that exclted electronic levels may be 
neglected if their energy is more than $4 K T$.

B. Translational Partition Function

The partition function per unft volume (l cc) for the translational motion of a molecule of mass $m$ having three degrees of translational freedom can be shown 19 to be

$$
q_{t r}=\frac{(2 \pi m k T)^{3 / 2}}{h^{3}}
$$

where $h$ is Planck's constant.

C. Vibrational Partition Function

The vibrational partition function for a diatomic molecule that has only a single internal vibration is approximated by the expression

$$
E_{v}=(n+1 / 2) h v_{0}
$$

where $n$ is an integer and $n \geq 0$. The vibrational partition function can be approximated as

$$
Q_{v}=\exp \left[-E_{0} / K T\right]
$$

when $h \nu_{0}>M T$, and where

$$
E_{0}=(1 / 2) h v_{0}
$$

represents the lowest vibrational energy possible for the molecule and is referred to as the zero-polnt energy, because it represents the residual energy of the molecule at $0^{\circ} \ddot{k}$.

For more complex molecules, assuming harmonic vibrations, the vibrational energy is 


$$
E_{v}=\sum_{i=1}^{j} \sum_{n=0}^{\infty}(n+1 / 2) h v_{i}
$$

where $v_{i}$ is the fundamental frequency of the Ith vibrational degree of freedom and there are $J$ vibrational degrees of freedom. For the ith degree of freedom

$$
\begin{aligned}
& \sum_{n=0}^{\infty} \exp \left[-(n+1 / 2) h v_{i} / K T\right] \\
&=\exp \left[-h v_{i} / 2 K T\right] \sum_{n=0}^{\infty} \exp \left[-n h v_{i} / K T\right] \\
&=\exp \left[-h v_{i} / 2 K T\right]\left[1-\exp \left(-h v_{i} / K T\right)\right]^{-1}
\end{aligned}
$$

so that the vibrational partition function for a polyatomic molecule, to the harmonic osclllator approximation, is

$$
a_{v}=\prod_{i=1}\left[\exp \left(-h v_{i} / 2 K T\right)\right]\left[1-\exp \left(-h v_{i} / K T\right)\right]^{-1}
$$

This can be written as

$$
a_{v}=\exp \left(-E_{0} / K T\right) \prod_{i=1}^{j}\left[1-\exp \left(-h v_{1} / K T\right)\right]^{-1}
$$

However since the zero-point energy is not independent of lsotopic substitution, it is convenient for our purpose. to write the vibrational partition in the form

$$
\begin{aligned}
Q_{v} & =\prod_{i=1}^{j}\left[\exp \left(h v_{i} / 2 K T\right)-\exp \left(-h v_{i} / 2 K T\right)\right]^{-1} \\
& =\prod_{i=1}\left[2 \sinh \left(h v_{i} / 2 K T\right)\right]^{-1}
\end{aligned}
$$


D. Rotational Partition Function

The allowed rotational states, for a diatomic molecule, will depend on whether or not the two nuclel are, identical. At temperatures at which the energy difference of adjacent rotational states 18 small compared to KT we can write an approximate molecular partition function

$$
Q_{\text {rot }}=\left(\frac{B \pi^{2} I K T}{\sigma h^{2}}\right)
$$

where I is the moment of Inertia perpendicular to the axis,

$$
I=\left(\frac{m_{1} m_{3}}{m_{1}+m_{2}}\right) r^{2}=\mu r^{2} .
$$

and $\sigma$, the symmetry number, equals 2 if the nuclei are Identical and is otherwise equal to 1 .

For polyatomic molecules, for comparatively high temperature, the rotational partition function is

$$
Q_{r o t}=\frac{\theta \pi^{3}\left(8 \pi^{3} A B C\right)^{1 / 2}(K T)^{3 / 2}}{\sigma h^{3}} \prod_{i}\left(2 S_{1}+1\right)
$$

where $A, B, C$ are the principal moments of inertia, $\sigma$ is equal to the number of indistingulshable ways of orienting the molecule in space, and $\Pi\left(2 s_{i}+1\right)$ ls the statistical spin factor for the nuclel.

E. Rate of Chemical Reaction

The rate of chemical reaction can be written as rate $=k C_{r}$ 
where $k$ is the specific rate constant, and $C_{r}$ is a functlon of reactant concentrations as experimentally observed. Then,

$$
c_{r}=k_{1} \Pi_{r} Q_{r}
$$

where $Q_{r}$ is the total partition function for the rth reactant, and $k_{1}$ is a constant whlch depends on stable and metastable configurations, whlch may or may not be reaction intermediates. The value of $k_{f}$ can be calculated statistically if structural parameters are known, by assuming these structures are in equilibrlum with the reactants. It is assumed that $K_{i}$ is independent of isotopic substitution; therefore, in the kinetic rate ratio formulation it would be cancelled out. 
5. Absolute Reaction Rate Theory

In Eq. (II-2I) $Q_{r}$ is the total molecular partition function per unit volume, which for a nonlinear reactant is

$$
Q_{r}=\left(\frac{2 \pi M_{r} K T}{h^{2}}\right)\left[\frac{\theta \pi^{2}\left(8 \pi^{3} A B C\right)^{1 / 2}(K T)^{3 / 2}}{\sigma_{r} h^{3}}\right] \prod_{i}^{3 n-6} \frac{1}{2 \sinh \left(\frac{U_{1}}{2}\right)}
$$

where $U_{i}=h c \omega_{1} / K T, w_{1}=$ fundamental frequencies of vibration. Eq. II -22 is evaluated by summing over all the energy states by allowing vibrational motlons about the stable configuration.

since the activated complex is unstable in one coordinate, the reaction coordinate, Eq. II -22 is invalid for the activated complex. This difficulty is avolded by Eyring 19 by restricting the activated complex by definition to a narrow range $\delta$ about the activation point.

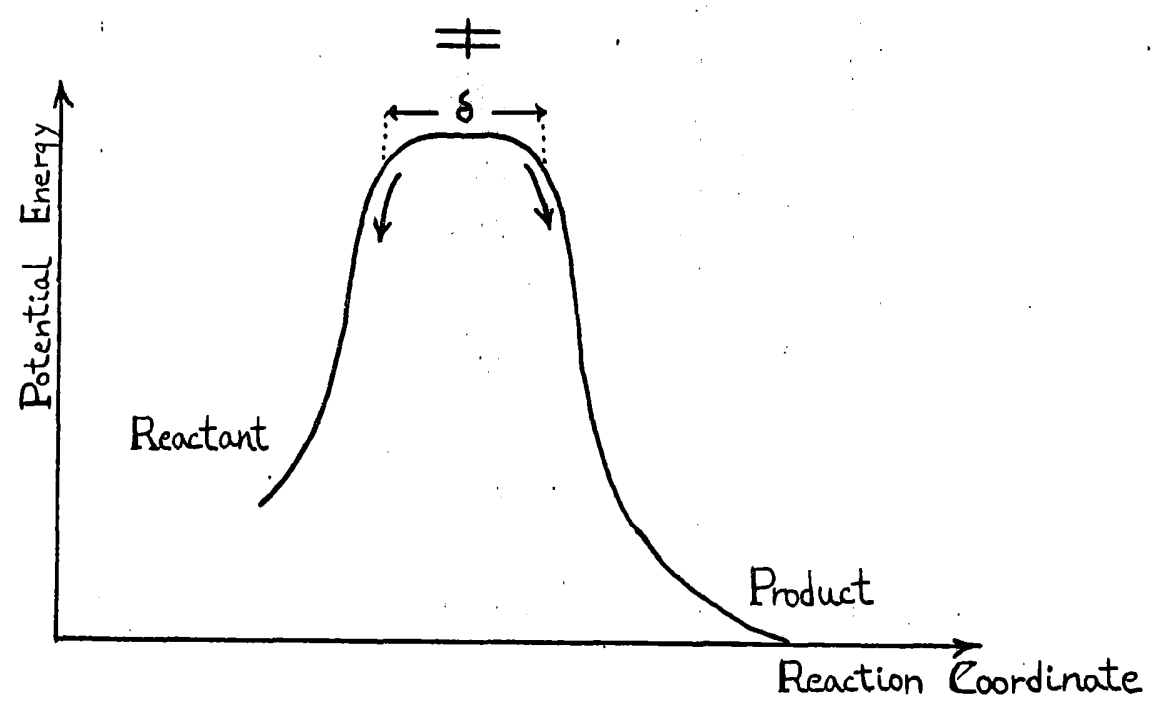

Fig. II -2

$\delta$-range of transition state. 
The transition state thus comprises all systems on the potential energy surface within a distance $\pm \delta / 2$ from a surface orthogonal to the reaction coordinate at the activation point. If we let $x$ be the reaction coordinate, the specific reaction rate constant 18

$$
k=K\left(\nu_{x} Q / \pi_{r} Q_{p}\right) \exp \left(-\Delta E_{0} / R T\right)
$$

where $K$, the probability of reflection at the energy barrler of systems which have sufficlent energy to surmount it, is the transmission coefficlent; $v_{x}$ is the frequency of motion $\ln x$; and $Q$ is the partition function of activated complex.

If motion in $x$ corresponds to a normal mode of vibration for the activated complex, then the $3 n-7$ other normal modes comprise motions within the transition state configuration, and the rate constant can be written as

$$
\begin{aligned}
& k=K\left(\frac{{ }^{\nu_{x}} Q_{x}^{\neq}}{\prod_{r} Q_{r}}\right)\left(\frac{2 \pi M^{*} K T}{h^{2}}\right)^{3 / 2} \cdot\left[\frac{8 \pi^{2}\left(8 \pi^{3} A^{*} B^{\ddagger} c^{\ddagger}\right)^{1 / 2}(K T)^{3 / 2}}{\sigma h^{3}}\right] x \\
& {\left[\prod_{1}^{3 n-7} \frac{1}{2 \sinh \left(\frac{U_{1}}{2}\right)}\right] \exp \left(-\Delta E_{0} / R T\right)}
\end{aligned}
$$

where $Q_{x}^{*}$ is the translational partition function corresponding to the motion of a particle in one-dimensional space.

Since we have assumed that all systems iying in the length $\delta$ shown in Fig. II -2 are actlvated complexes, we 
can further asoume that the concentration of activated complexes depends only upon the potential energy within the activated state. Therefore the calculated rate will be unchanged if the partition function which has a maximum in $x$ is replaced by one for which potential energy is constant in $x$ or by one which has a minimum in $x$.

The translational motion of a particle of mass $\mathrm{m}$ is one-dimensional box of length $\delta$ can be described by the translational partition function

$$
Q_{t r}=\frac{(2 \pi m k T)^{1 / 2}}{h} \delta
$$

so if the "effective mass"* is $m_{0}$ the partition function assoclated with motion in $x$ of the activated complex is given by

$$
Q_{x}^{*}=\left[\frac{2 \pi m_{0} K T}{h^{2}}\right]^{1 / 2} \delta
$$

The frequency in $\times 18$

$$
\begin{aligned}
v_{x} & =\frac{\bar{x}}{\delta} \\
& =\frac{1}{\delta} \times\left[\frac{\int_{0}^{\infty} \exp \left(\frac{-m_{0} \dot{x}^{2}}{2 K T}\right) \dot{x} d \dot{x}}{[+\infty} \exp \left(\frac{-m_{0} x^{2}}{2 K T}\right) d \dot{x}\right. \\
& =\frac{1}{\delta} \times\left[\frac{K T}{2 \pi m_{0}}\right]
\end{aligned}
$$

* In the case of two "osclllator" system, the effective mass 18 the reduced mass. 
In the numerator we take one-half of the integral

$$
\int_{-\infty}^{+\infty} \exp \left(\frac{-m_{0} \dot{x}^{2}}{2 k T}\right) \dot{x} d \dot{x},
$$

since we are interested only in those motions which lead

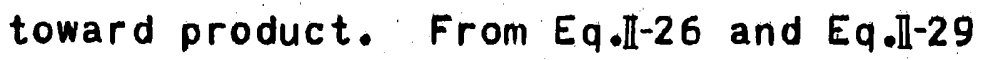

$$
\nu_{x} Q_{x}^{\ddagger}=\frac{1}{\delta}\left(\frac{K T}{2 \pi m_{0}}\right)^{1 / 2}\left(\frac{2 \pi m_{0} k T}{h^{2}}\right)^{1 / 2} \delta=\frac{K T}{h}
$$

Thus we see that the actual value of $\delta$ is immaterlal. Hence, the specific reaction rate can be written

$$
\begin{aligned}
& k=\left(K \frac{K T}{h}\right)\left(\pi Q_{r}\right)^{-1}\left(\frac{2 \pi M K T}{h^{2}}\right)^{3 / 2}\left[\frac{\theta \pi^{2}\left(\theta \pi^{3} A^{*} \theta^{*} C^{*}\right)^{1 / 2}(K T)^{3 / 2}}{\sigma h^{3}}\right] \\
& \quad x\left[\prod_{1}^{3 n-7}\left(2 \sinh \frac{U_{1}}{2}\right)^{-1}\right] \exp \left(-E_{0} / R T\right)
\end{aligned}
$$

The above expression can be derived by alternative methods. As we have mentioned in the previous sections that the total partition function can be separated into contributions corresponding to translational, rotational, vibrational, and electronic energies. The partition function for vibrational motion per normal mode 18 given by

$$
Q_{v}=[1-\exp (-h v / K T)]^{-1} \exp [h v / 2 K T]
$$

If one degree of the vibrational energy of the activated complexes corresponds to the very loose vibration, then it can be considered as the reaction coordinate and 


$$
\operatorname{L~}_{\nu \rightarrow 0} Q_{v}=\nu \stackrel{L_{\nu}}{\rightarrow}[1-\exp (-n \nu / k T)]^{-1}
$$

According to Maclaurin series expansion

$$
\exp (-h \nu / K T)=\sum_{n=0}^{\infty} \frac{(-h \nu / K T)^{n}}{n !}
$$

when $|\mathrm{hv} / \mathrm{kT}|<\infty$,

then $L_{\nu \rightarrow 0}\left[-\sum_{n=0}^{\infty} \frac{(-h \nu / k T)^{n}}{n !}\right]=\left[1-L_{\nu \rightarrow 0} \sum_{n=0}^{\infty} \frac{(-h \nu / k T)^{n}}{n !}\right]^{-1}$

If we omit the square and higher terms in Eq. II-34, Eq. II -27 becomes

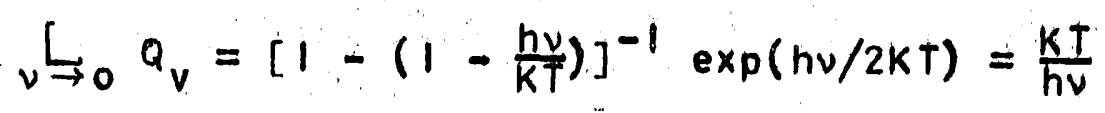

Hence the equilibrlum constant between the reactants and the activated complexes can be written as

$$
K^{*}=\frac{\left[x^{*}\right]}{r[r]}\left(\frac{K T}{h \nu}\right)\left[\frac{Q^{*}}{\pi^{2} Q_{r}} \exp \left(-\Delta E_{0} / R T\right)\right]
$$

where $Q_{\neq}$is the partition function of activated complexes less one degree of vibrational freedom, and $[r]$ is the concentration of reactants. By rearranging Eq'. II -37 , we have

$$
\left.\nu\left[x^{\neq}\right]=\pi_{r}[r]\left(\frac{K T}{h}\right) \underset{r}{\pi Q_{r}} \exp \left(-\Delta E_{0} / R T\right)\right]
$$

The left-hand slde of Eq. II-40 can be divided by the concentration of reactants to give the rate constant of reaction 


$$
k=\left(\frac{K T}{h}\right)\left[\frac{Q_{*}}{r Q_{r}} \exp \left(-\Delta E_{0} / R T\right)\right]
$$

Since not every actluated complex reaching the top of the potential energy barrier lo converted into reaction product we introduce the "transmission coefflclent" $t$. Then Eq. II -39 can be written as

$$
k=k \frac{K T}{h}\left[\frac{Q_{\neq}}{\pi} Q_{r} \exp \left(-\Delta E_{0} / R T\right)\right]
$$

This result is identical with that obtained by Eyring method in Eq. II-3I.

In the quantum mechanical treatment of reaction vibration, a slightly different expression from Eq. II-18 was obtained:

$$
\nu_{q} Q_{q}=\nu_{q}\left[2 \sinh \left(\frac{U_{q}}{2}\right)\right]^{-1}
$$

However, if we assume $v_{q}=\left|v_{x}\right|$ and $\frac{h v_{q}}{k T}<1$, then

$$
\nu_{q} Q_{q}=\left(\frac{K T}{h}\right)\left[1+\frac{U_{q}^{a}}{24}\right]^{-1}
$$

The difference between the two is approximately the quantum mechanical tunneling factor. 20

The expression for the specific rate constant, which will be employed through the rest of this discussion, is obtained. 


$$
\begin{aligned}
& k=\left(k \frac{K T}{h}\right) \prod_{r}^{\pi}\left(\frac{2 \pi M_{r} K T}{h^{2}}\right)^{3 / 2} \frac{\theta \pi^{3}\left(\theta \pi^{3} A_{r}{ }_{r} C_{r}\right)^{1 / 2}(K T)^{3 / 2}}{\sigma_{r} h^{3}} \\
& \left.x_{1}^{3 n-6} \frac{1}{2 \sinh \left(\frac{U}{2}\right)}\right\}^{-1}\left(\frac{2 \pi M^{*} K T}{h^{3}}\right)^{3 / 2}\left[\frac{8 \pi^{2}\left(B \pi^{3} A^{*} B^{*} C^{*}\right)^{1 / 2}(K T)^{3 / 2}}{\sigma_{r}^{*} h^{3}}\right] \\
& \times \prod_{1}^{3 n-7} \frac{1}{2 \sinh \left(\frac{U_{1}}{2}\right)}
\end{aligned}
$$


6. Isotope Effects

\section{A. Isotope Effects on Potential-Energy Surfaces}

The calculation of the influence of lsotople mase on the rate of reaction, it is clear, requires a set of very detalled descriptions of the roles of the individual atoms and the forces exerted by the electron cloud. It is also clear that in order to predict the reaction rate and the isotope effect of a certain reaction accurately, detalled information about the transition state is required. The absolute reaction rate theory thus has proved to be a better method than others for this purpose at the present stage. However, in practical cases, this theory is limited by our incomplete knowledge of the actual transition state and the impossiblifty, at present, of making a quantummechanical calculation accurate enough for any but the simplest systems. A qualitative agreement between the theoretical prediction and experimental data is, therefore, generally sufficlent.

Theoretical models, In general, are based upon the presently avallable knowledge about stable molecules. Most bonds which are not directly involved in the reaction will behave normally as to length and force constant. For bonds which are ruptured in the reaction, the length will generally be increased and the force constant weakened if the corresponding mode of vibration has not disappeared completely as a consequence of the 
transformation of the corresponding vibrational coordinate into the coordinate of decomposition. In general, as a result of the weakening of the corresponding vibrational coordinate, we assume that the requency of thls vibration is zero.

In ordinary calculation of the reaction rates we know very little about the transmission coefflclent and the amount of tunneling through the potential energy barrler. However, in the absolute rate theory the tunneling contribution is generally negliglble. And the transmission coefficlent is not very sensitive to lsotoplc mass and will consequently cancel in the kinetic lsotope rate ratio.

By defintition, the isotopes are two forms of an element differing only in the number of neutrons in the nucleus but not in any ordinary chemlcal properties. since the electronic structures of two lsotopes of the same element are identical, the mass of the nucleus enters the calculations only via the "effective mass" of the system of nucleus plus electrons. Hence the forces which hold the atoms together wlll be nearly independent of changes in the masses of the atomic nuclei caused by isotopic substitution. Then we can say that the potential-energy surface and hence the interatomic distances and the vibrational force constants could be treated as invariant under lsotoplc substitution, with an accuracy 
which is sufflcient for our present purpose. But zero-point energy (and thus activation energy) and $v_{x}$ depend upon vibrational frequencles and thus on masses. A potential-energy diagram for the lsotoplc substitution of light molecules by heavier molecules lo shown in FIg. III-I. 


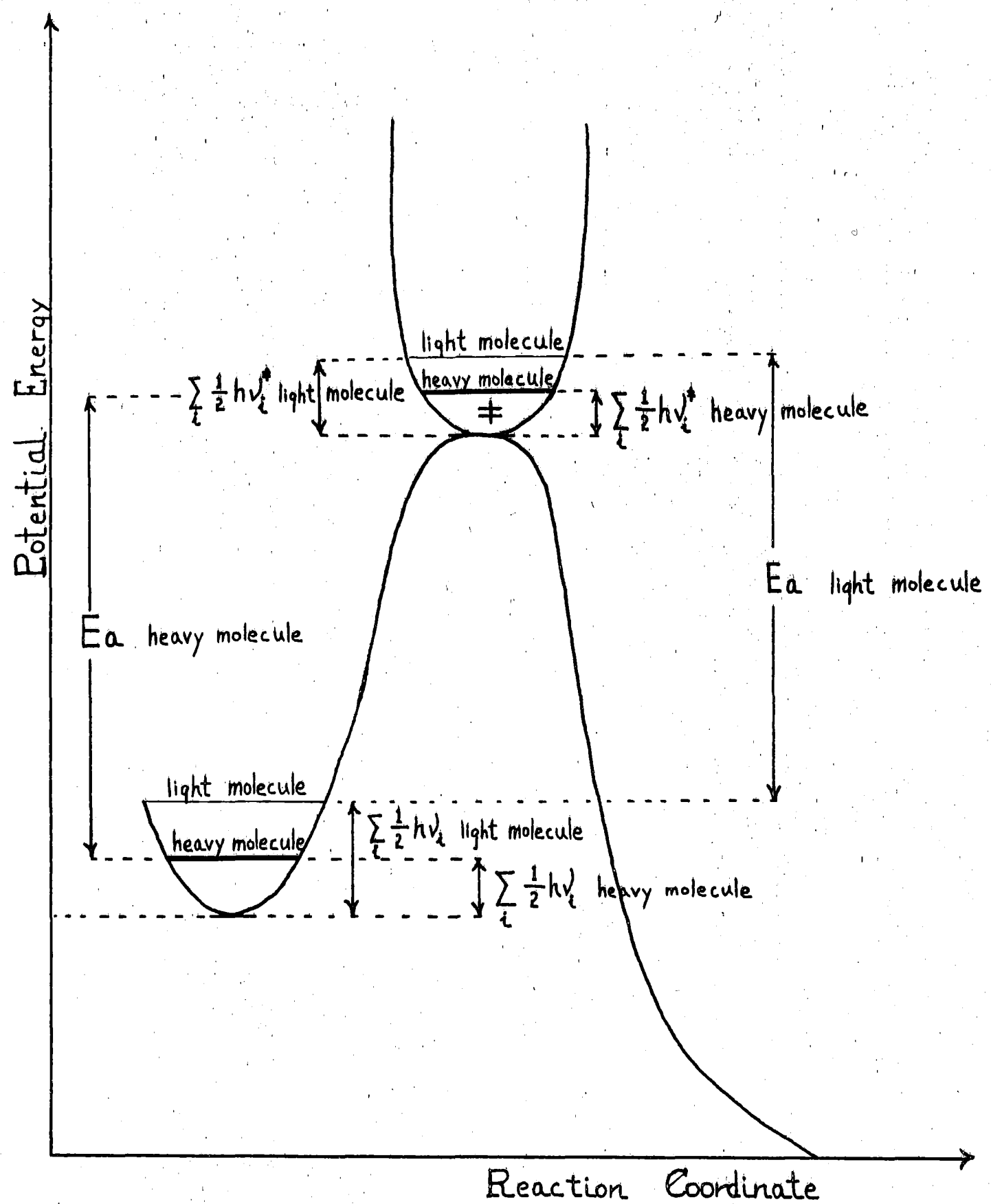

Fig. III-I Potential-Energy diagram for isotope effect. 
B. Application of Rate Theory to Isotope Effects

$$
\begin{gathered}
\text { Consider the unimolecular decomposition } \\
x=x^{\neq}=\text {products, }
\end{gathered}
$$

in which the valldity of QET is assumed, then the rate constant for this reaction can be written in the form of $\mathrm{Eq}$. II -45 :

$$
\begin{gathered}
k=\left(k \frac{K T}{h}\right)\left[\left(\frac{2 \pi M_{r} K T}{h^{2}}\right)^{3 / 2} \frac{8 \pi^{2}\left(8 \pi^{3} A r^{B} r^{C}\right)^{1 / 2}}{k T)^{3 / 2}}\right. \\
\left.\times \prod_{1}^{3 n-6} \frac{1}{2 \sinh \left(\frac{U_{1}}{2}\right)}\right]^{-1}
\end{gathered}
$$

$$
\times\left(\frac{2 \pi M^{*} K T}{h^{2}}\right)^{3 / 2} \frac{\theta \pi^{3}\left(B \pi^{3} A^{*} B^{*} C^{*}\right)^{1 / 2}(K T)^{3 / 2}}{\sigma^{*} h^{3}} \prod_{1}^{3 n-7} \frac{1}{2 \ln h\left(\frac{1}{2}\right)}
$$

$(11-44)$

If we replace one atom in $x$ by a lighter lsotope, the reaction is written as:

$$
x^{\prime}=x^{i \neq}=\text { Products, }
$$

and the rate constant is

$$
\begin{aligned}
& k^{\prime}=\left(k^{*} \frac{K T}{h}\right)\left[\left(\frac{2 \pi M_{r}^{\prime} K T}{h^{3}}\right)^{3 / 2} \frac{8 \pi^{3}\left(8 \pi^{3} A_{r}^{\prime} B_{r}^{\prime} C_{r}^{\prime}\right)^{1 / 2}(K T)^{3 / 2}}{\sigma_{r}^{\prime} h^{3}}\right. \\
& \left.x_{1}^{3 n-6} \frac{1}{2 \sinh \left(\frac{1}{2}\right)}\right]^{-1}\left(\frac{2 \pi M^{* 1} K T}{h^{3}}\right)^{3 / 2} \frac{\theta^{\pi 2}\left(B \pi^{3} A^{* 1} B^{* 1} C^{* 1}\right)^{1 / 2}(K T)^{3 / 2}}{\sigma h^{3}}
\end{aligned}
$$

$$
\prod_{1}^{3 n-7} \frac{1}{2 \sinh \left(\frac{\pi}{2}\right)}
$$


By comparing Eq. II -44 and Eq. II-45, we have,

$$
\begin{aligned}
& \left.\frac{k^{\prime}}{k}=\left(\frac{K^{\prime}}{K}\right)\left[\left(\frac{M_{r}^{\prime}}{M_{r}}\right)^{3 / 2}\left(\frac{\sigma_{r}}{\sigma_{r}}\right) \frac{A^{\prime} B_{r}^{\prime} C_{r}^{1 / 2}}{A_{r} B_{r} C_{r}}\right)_{1}^{3 n-6} \frac{\sinh \left(\frac{U_{1}}{2}\right)}{\sinh \left(\frac{U_{1}}{2}\right)}\right]^{-1} \\
& \times\left(\frac{M^{* 1}}{M^{*}}\right)^{3 / 2}\left(\frac{\sigma^{*}}{\sigma^{* T}}\right)\left(\frac{\left.A^{* 1} B^{* 1} C^{* 1}\right)^{1 / 2} B^{*} C^{*}}{\prod_{1}^{3 n-7}} \frac{\sinh \left(\frac{U_{1}^{*}}{2}\right)}{\sinh \left(\frac{U_{1}^{*}}{2}\right)}\right.
\end{aligned}
$$

If we rearrange Eq. II-46 and assume that the transmlsslon-coefficlent cancel out, we get

$$
\begin{aligned}
& \frac{k^{\prime}}{k}=\left(\frac{M_{r}}{M_{r}} \times \frac{M^{* 1}}{M^{*}}\right)^{3 / 2}\left(\frac{\sigma_{r}^{\prime}}{\sigma_{r}} \times \frac{\sigma^{*}}{\sigma^{* 1}}\right)\left(\frac{A_{r}^{B} C_{r}}{A_{r}{ }^{B} C_{r}} \times \frac{A^{*} \cdot B^{* 1} C^{* 1}}{A^{*} B^{*} C^{*}}\right)^{1 / 2} \\
& \times \prod_{1}^{3 n-6} \frac{\sinh \left(\frac{U_{1}}{2}\right)}{\sinh \left(\frac{U_{1}}{2}\right)} \quad \frac{3 n-7}{1} \frac{\sinh \left(\frac{U_{1}^{*}}{2}\right)}{\sinh \left(\frac{U_{1}^{*}}{2}\right)}
\end{aligned}
$$

By multiplying both sides of Eq. II -47 by $\left(\sigma^{* 1} \sigma_{r}^{1}\right) /\left(\sigma^{*} \sigma_{p}^{p}\right)$ we arrlve our general expression:

$$
\begin{aligned}
& \left(\frac{k^{\prime}}{k}\right)\left[\frac{\sigma_{r} \sigma^{* 1}}{\sigma_{r} \sigma^{*}}\right]=\left(\frac{M_{r}}{M_{r}} \times \frac{M^{* 1}}{M^{*}}\right)^{3 / 2} \cdot\left(\frac{A_{r}{ }^{B} r^{C} r}{{ }_{r} B_{r} C_{r}} \times \frac{A^{*} B^{* 1} C^{* 1}}{A * B^{*} C^{*}}\right)^{1 / 2} \\
& x \prod_{i}^{3 n-6} \frac{\sinh \left(\frac{U_{i}}{2}\right)}{\sinh \left(\frac{U_{1}}{2}\right)} \quad \prod_{i}^{3 n-7} \frac{\sinh \left(\frac{U_{i}^{*}}{2}\right)}{\sinh \left(\frac{U_{i}^{*}}{2}\right)}
\end{aligned}
$$

The above expression can be simplifled by the use of identies derlued from the Redilch-teller product rule 21. 
These same identities can be obtained by equating classlcal partition functions with the high-temperature limit of quantium mechanlcal partition functions, as suggested by Blgeleisen and Meyer ${ }^{5}$. The expression derlved for the reactants is

$$
\left(\frac{m^{1}}{m}\right)^{1 / 2}\left(\frac{M}{M}\right)^{3 / 2} \cdot\left(\frac{A B C}{A-B T}\right)^{1 / 2} \prod_{i}^{3 n-6} \quad \frac{U}{U_{i}}=1
$$

for the activated complex when motion in' the reaction coordinate is considered as a vibration is

$$
\left(\frac{m^{1}}{m}\right)^{1 / 2}\left(\frac{M^{*}}{M \neq T}\right)^{3 / 2}\left(\frac{A^{*} B^{*} C^{*}}{A^{*} T C^{* T}}\right)^{1 / 2} \prod_{i}^{3 n-6} \cdot \frac{U_{1}^{*} i}{U_{1}^{*}}=1 ;
$$

and that for the activated complex where motion in the reaction coordinate is considered as a translation is

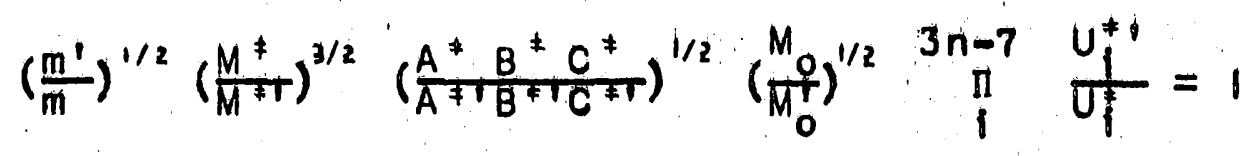

If we combine $E q . I I-49$ and $E q . I I-5 I$ we get

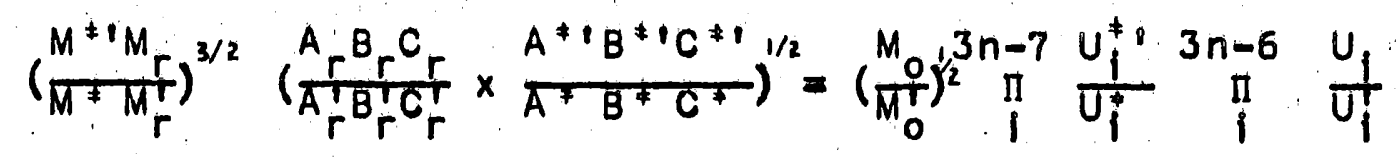

By combining Eq. II -48 and $E q . I I-52$ we have

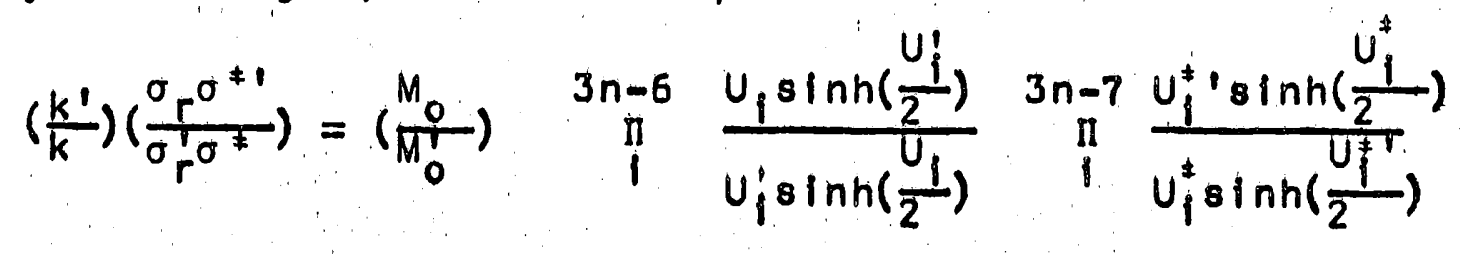

or 


$$
\left(\frac{k^{\prime}}{k}\right)\left(\frac{\sigma_{r} \sigma^{\prime}}{\sigma_{r} \sigma}\right)=\left(\frac{v_{x}^{\prime}}{v_{x}}\right) \prod_{1}^{3 n-6} \frac{U_{1} \sinh \left(\frac{U_{1}}{2}\right)}{U_{1} \sinh \left(\frac{U_{i}}{2}\right)} \prod_{1}^{3 n-7} \frac{U_{1}^{\prime} \sinh \left(\frac{U_{1}}{2}\right)}{U_{1} \sinh \left(\frac{U_{1}}{2}\right)}
$$

where $\nu_{x}$ is the average frequency of motion in the reaction coordinate.

In the case of Intramolecular lsotope effects, the reactants are ldentical for the palr of reactions, and $\left(Q_{r} / Q_{r}^{\prime}\right)=1$. Then, Eq. II-53 or Eq. II-54 can be written $\mathbf{a} 8$

$$
\begin{aligned}
\left(\frac{k^{\prime}}{k}\right)\left(\frac{\sigma_{r^{\prime}} \sigma_{r^{\prime}}^{\prime}}{r^{\prime}}\right) & =\left(\frac{v_{x}^{\prime}}{v_{x}}\right) \frac{3 n-7}{\prod_{1}} \frac{U_{1}^{\prime} \sinh \left(\frac{U_{1}}{2}\right)}{U_{1} \sinh \left(\frac{U_{1}}{2}\right)} \\
& =\left({\frac{M_{0}}{M_{0}^{+}}}_{0}^{3 n-7} \prod_{1}^{3 n} \frac{U_{1}^{\prime} \sinh \left(\frac{U_{1}}{2}\right)}{U_{1} \sinh \left(\frac{U_{1}}{2}\right)}\right.
\end{aligned}
$$

In Eq. II-53 or II -54 it is obvious that the first term of the right-hand side is the "Temperature-Independent Factor " (TIF), and the second term of the right-hand side is the "Temperature-Dependent Factor" (TDF). Thus the theoretical expressions for rate constant ratios of lsotoplc reactions can be written in the general form

$$
\frac{k^{\prime}}{k}=(T I F) \quad(T D F)
$$

The temperature-dependent factor is a function of the frequencles of motions orthogonal to the reaction coordinate. In the case of intermolecular reactions, it 
depends also upon the fundamental frequencles of the reactants. The temperature-independent factor, $\left(\nu_{x} / \nu_{x}\right)$ has been generalily consldered as the equivalent of $\left(m_{0} / m_{0}^{l}\right)$, which in actual computation Involves considerable uncertalnty. Thls calculated uncertalnty, however, would not Influence the temperature-dependent factor. This problem has, in 1958, been reviewed by Blgelelsen and Wolfsberg, 22 and they have given a partlcular formula 23 for the temperature-independent function to be used in the case of three-centered reactions. 24 In Eq. II-55 the term $U_{1}$, as defined, is equal to

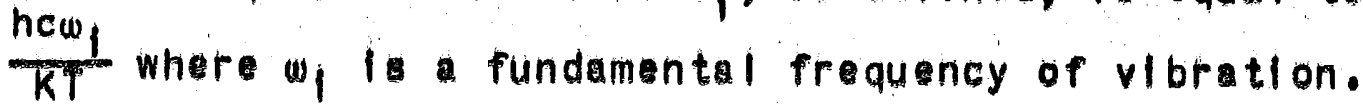
In order to calculate the ratio, two sets of fundamental frequencles must be known. Therefore, a detalled development and geheral method of calculating these sets of fundamental frequencles are desirable. The following chapter is devoted to thls purpose. 


$$
\text { Chapter III - Molecular Vibrations }
$$

1. Separation of Energies 41

2. Coordinate Systems of Small Vibrations 47

A. Generallized Coordinate System (GCS)

Kinetic Energy in GCS

Potential Energy in GCS

Coefficients of the Energy Equations in GCS

Equations of Motion in GCS

B. Mass-Wel ghted Cartesian Coordinates (MWCC)

and the Principal Axis Transformation

Mass-Welghted Cartesian Coordinates

The Principal Axis Transformation

c. Normal Coordinates and Normal Modes of

Vibration

D. Internal Coordinate System

Description of Internal Coordinate System

G Matrlx and the Secular Equation in Terms of

Internal Coordinates 


\section{Chapter III - Molecular Vibration}

Throughout this investigation the atoms are treated as point masses connected by forces which keep the atoms near their equilibrlum positions. The electronlc effects are neglected. 16 The rigorous and logical way to begin the treatment of molecular vibrations, is to prove that when the proper coordinate system is used, the total energy of a molecule can be approximately separated Into three components, translational energy, rotational energy, and vibrational energy. 
1. Separation of Energies

The coordinate system used here is construclted as follows: The three Cartesian coorllinates whose origin Is located at the center of mass of the molecule; the three Eulerian angles of a rotating system of Cartesian coordinates, the axes of which coincide with the principal axes of inertia of the undistorted molecule; and the Cartesian coordinates of the atoms with respect to the rotating coordinate system.' The system whose or 1gin is located at the center of mass of the molecule is denoted by $I$, and the other one is denoted by $S$. If we have a partlcle $P$ in the space of $S$, there results three types of movements; relative motion, $P / S$, is the motion of $P$ with respect to S; leading motion, S/I, is the motion of $S$ with respect to I; absolute motion, $P \mid I$, is the motion of $P$ with respect to I. If the three coordinates of I are $x, y, z$ with unit vectors $\hat{i}, \hat{j}, \hat{k}$, respectively, and the coordinates of $s$ are $u, v, w$ with unit vectors $\hat{l}, \hat{m}, \hat{n}$, we have defined the position of the point mass $P$. And we can denote $P$ as a function of six coordinates: $P(x, y, z, u, v, w)$. The relative position is shown in Fig. III-I. 


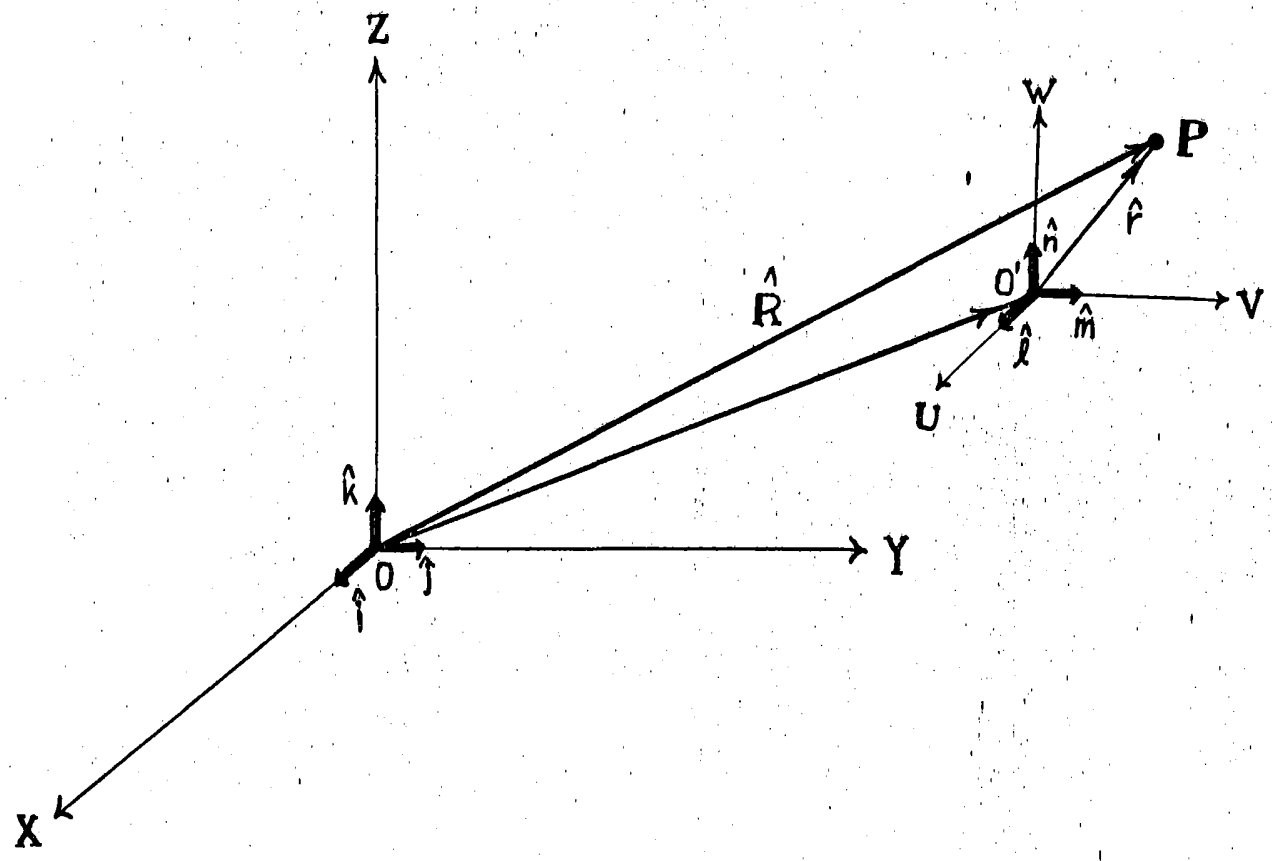

Fig. III-I The relative position of $S$ and I systems in three dimensional space.

With reference to Fig. III-l it is clear that

$$
\hat{O P}=\hat{O O}+\hat{O}^{\prime} P=\hat{O O}+(u \hat{I}+v \hat{m}+\hat{n}) \quad(\text { III-I) }
$$

By differentiating Eq. III-l with respect to time, $t$, we get the absolute velocity $V_{a b}$ in terms of leading veloclty $V_{l}$, and relative velocity, $V_{r}$.

$$
\begin{aligned}
V_{a b} & =\frac{d \hat{O P}}{d t}=\frac{d \hat{O O O}^{\prime}}{d t}+\frac{d \hat{O}^{\prime} p}{d t} \\
& =\frac{d \hat{O O}^{\prime}}{d t}+\frac{d}{d t}[u \hat{\imath}+v \hat{m}+w \hat{n}] \\
& =\frac{d \hat{O O}^{\prime}}{d t}+\left(u \frac{d \hat{l}}{d t}+\hat{i} \frac{d u}{d t}\right)+\left(v \frac{d \hat{m}}{d t}+\hat{m} \frac{d v}{d t}\right)+\left(w \frac{d \hat{n}}{d t}\right. \\
& \left.=\hat{v}_{l}+\hat{v}_{r}+\hat{n} \frac{d w}{d t}\right)
\end{aligned}
$$


where $v_{\ell}=\frac{d \hat{O O}}{d t}+\left(u \frac{d \hat{l}}{d t}+v \frac{d \hat{m}}{d t}+w \frac{d \hat{n}}{d t}\right)$

43

$$
v_{r}=\frac{d u}{d t} \hat{\imath}+\frac{d v}{d t} \hat{m}+\frac{d w}{d t} \hat{n}
$$

(III -3)

Now, let us take a look at system S. (FIg. III -2)

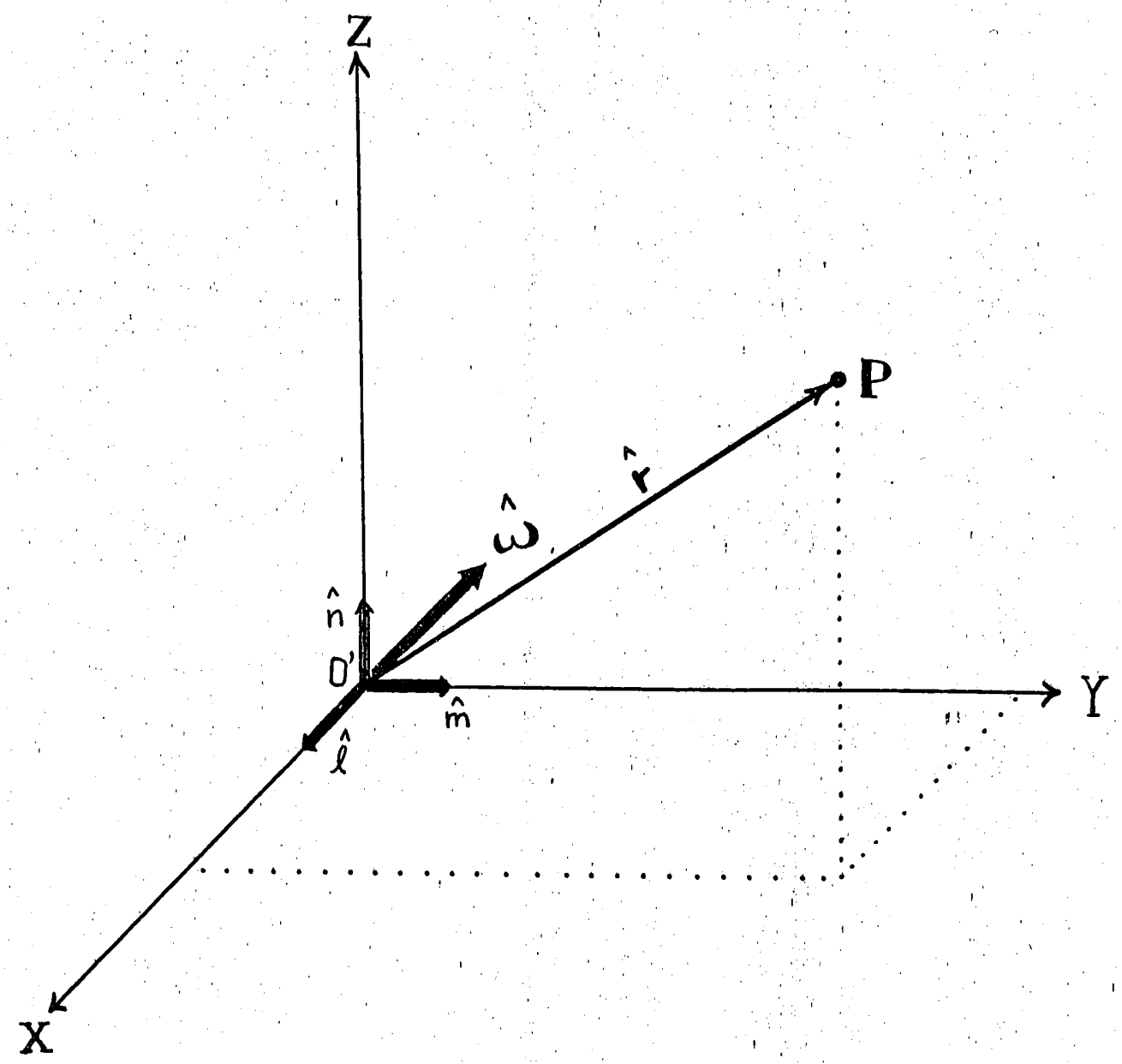

Fig. III -2. S -system 
Since $\hat{l}$ and $\hat{i}$ are orthogonal then

$$
\hat{i}=a \hat{n}+b \hat{n}
$$

where $a$ and $b$ are constants.

Consider

$$
\begin{aligned}
& \hat{m} \cdot \hat{i}=a \hat{m} \cdot \hat{m}+b \hat{m} \cdot \hat{n}=a \\
& \hat{n} \cdot \hat{i}=a \hat{n} \cdot \hat{m}+b \hat{n} \cdot \hat{n}=b
\end{aligned}
$$

By substituting Eq. III -6 and III-7 into Eq. III-5,

we get

$$
\hat{i}=(\hat{m} \cdot \hat{i}) \hat{m}+(\hat{n} \cdot \hat{i}) \hat{n} \text {. }
$$

since $\hat{\imath} \cdot \hat{n}=0$, we know that

$$
\begin{aligned}
& \hat{i} \cdot \hat{n}+\hat{n} \cdot \hat{i}=0 \\
\therefore \quad & \hat{n} \cdot \dot{\hat{l}}=-\hat{i} \cdot \hat{n}
\end{aligned}
$$

By combining $E q$. II $-B$ and $E q$. III-9,

$$
\begin{aligned}
\hat{i} & =(\hat{m} \cdot \hat{i}) \hat{m}-(\hat{i} \cdot \hat{n}) \hat{n} \\
& =(\hat{i} \cdot \hat{m})(\hat{n} \times \hat{i})+(\hat{n} \cdot \hat{i})(\hat{m} \times \hat{i})+(\hat{m} \cdot \hat{n})(\hat{I} \times \hat{i}) \\
& =[(\hat{i} \cdot \hat{m}) \hat{n}+(\hat{n} \cdot \hat{i}) \hat{m}+(\hat{m} \cdot \hat{n}) \hat{i}] \times \hat{i} \\
& =\hat{\omega} \times \hat{i}
\end{aligned}
$$

where $\hat{\omega}$ is the angular velocity of the particle $P$.

since $\hat{l}, \hat{m}$, and $\hat{n}$ are cyclic permutative, we can write

down

$$
\begin{aligned}
& \hat{i}=\hat{i} \times \hat{i} \\
& \dot{\hat{m}}=\hat{\omega} \times \hat{m} \\
& \dot{\hat{n}}=\hat{n} \times \hat{n}
\end{aligned}
$$


If we combine Eqs. III-10 and III-3, we get

$$
\begin{aligned}
\hat{v}_{\ell} & =\dot{\hat{O O}}^{\prime}+\hat{\omega} \times(u \hat{\imath}+v \hat{m}+w \hat{n}) \\
& =\dot{\hat{O}}++\hat{\omega} \times \hat{O P}
\end{aligned}
$$

So the absolute velocity (Eq. III-2) can be written

$$
\hat{v}_{a b}=\left(\hat{O O O}^{\prime}\right)+(\hat{\omega} \times \hat{O P})+\hat{v}_{r}=\hat{R}+\hat{\omega} \times \hat{r}+\hat{v}_{r}(\text { III-12) }
$$

The kinetic energy $T$ of the whole molecule, therefore, can be written

$$
\begin{aligned}
2 T= & \dot{\hat{R}}^{2} \sum_{i} m_{i}+\sum_{i}(\hat{\omega} \times \hat{r}) \cdot(\hat{\omega} \times \hat{r})+\sum m_{1} \hat{v}_{i}^{z} \\
& +2 \dot{\hat{R}} \cdot \hat{\omega} \times \sum_{i} m_{i} \hat{r}_{i}+2 \dot{\hat{R}} \cdot \sum_{i} \hat{v}_{i}+2 \hat{\omega} \cdot \sum\left(m_{i} \hat{r}_{i} \times \hat{v}_{i}\right)
\end{aligned}
$$

Since the origin, 0 , is the center of gravity of the whole molecule, at every instant it must be true that

$$
\sum_{i} \hat{r}_{i}=0
$$

and $\sum_{i} \hat{r}_{i}=\sum_{i} m_{i}\left[\left(\hat{w} \times \hat{r}_{i}\right)+\hat{v}_{i}\right]=0$

$$
\sum_{1} \hat{m}_{1} \hat{v}_{1}=0
$$

Equation III-13 becomes

$$
\begin{aligned}
& 2 T=\hat{\hat{R}}^{2} \sum_{i} m_{i}+\sum m_{i}(\hat{w} \times \hat{r}) \cdot(\hat{\omega} \times \hat{r})+\sum_{i} m_{i} \hat{V}_{i}^{2}+2 \hat{\omega} \cdot \sum m_{i}\left(\hat{r}_{i} \times \hat{V}_{i}\right) \\
& =\text { translational energy + rotational energy }+ \\
& \quad \text { vibrational energy + Corlolis energy } 26
\end{aligned}
$$


Thus far we have proved that the total energy of a molecule can be approximately separated into transiational, rotational, vibrational, and Corlolis energies. Hereafter, the discussion will be centered upon the vibrational energy and related functions. 
2. Coordinate Systems

A. Generalized Coordinate system

If a set of properly defined coordinates, as stated in the previous section, is chosen, the vibration of a molecule can be treated as a conservative small osclllation system. Let us consider a polyatomic molecule, as a conservative'system, which can be described in terms of a set of generalized coordinates, $q_{k}$, and the time, $t$. If the molecule possesses $n$ degrees of freedom, then $k=1,2, \ldots \ldots, n$, We specify that a configuration of stable equilibrium exists for the system, and that at equilibrlum the generallzed coordinates have values $a_{k}$. In such a configuration Lagrange's equation

$$
\frac{\partial L}{\partial q_{k}}-\frac{d}{d t} \frac{\partial L}{\partial q_{k}}=0 \quad k=1,2, \ldots, n
$$

will be satisfled by

$$
q_{k}=\dot{q}_{k 0} ; \dot{q}_{k}=0 ; \quad \ddot{q}_{k}=0 ; k=1,2, \ldots, n
$$

Now, at the least every nonzero term of the form $\left(\frac{d}{d t} \frac{\partial L}{\partial \dot{q}_{k}}\right)$ must contain efther $\dot{q}_{k}$ or $\ddot{q}_{k}$, so that all such terms vanish at equilibrium. Therefore, from Lagrang's equation, we have

$$
\left.\frac{\partial L}{\partial q_{k}}\right|_{0}=\left.\frac{\partial T}{\partial q_{k}}\right|_{0}-\left.\frac{\partial U}{\partial q_{k}}\right|_{0}=0
$$

where the subscripts, 0 , designates that the quantity is evaluated at equillbrium. 
It is assumed that the transformation equations defining the generalized coordinates of the system, $q_{1}, \ldots ., q_{n}$, do not involve the time explicity. Thus, time-dependent constraints are to be excluded. Then potential energy is a function of position only, and the kinetic energy is a function of velocity only.

Kinetic Energy in Generalized Coordinate system

In a rectangular coordinate system, the kinetic energy 18 given by

$$
T=1 / 2 \sum_{\alpha=1}^{n} \sum_{i=1}^{3} m_{\alpha} \dot{x}_{\alpha, i}^{2}
$$

If we adopt a set of generalized coordinates explicltiy including the time, then the set of transformations connecting the rectangular system and the generalized system can be written as

$$
x_{\alpha, i}=x_{\alpha, i}\left(q_{1}, q_{z}, \ldots . q_{n}, t\right)
$$

where $\alpha=1,2, \ldots \ldots, n ; \quad i=1,2,3 ;$ then

$$
x_{a, i}=x_{\alpha, i}\left(q_{j}, t\right)
$$

where $\mathrm{j}=1,2, \ldots, \mathrm{h}$.

The generallzed velocity is

$$
\dot{x}_{\alpha, i}=\dot{x}_{\alpha, i} \quad\left(q_{j}, \dot{q}_{j}, t\right) \text {. }
$$

We may also write the inverse traniformation as

$$
\begin{aligned}
& q_{j}=q_{j}\left(x_{\alpha}, f, t\right) \\
& \dot{q}_{j}=\dot{q}_{j}\left(x_{\alpha}, f, \dot{x}_{\alpha}, t, t\right)
\end{aligned}
$$


Eq: II -20 can be written as

$$
\dot{x}_{a, i}=\sum_{j=1}^{h} \frac{\partial x_{a \cdot i}}{\partial q_{j}} \dot{q}_{j}+\frac{\partial x_{\alpha \cdot i}}{\partial t}
$$

Then,

$$
\begin{aligned}
\left(\dot{x}_{\alpha, 1}\right)^{2} & =\left(\sum_{j=1}^{h} \sum_{k=1}^{h} \frac{\partial x_{\alpha}, 1}{\partial q_{j}} \frac{\partial x_{\alpha}, 1}{\partial q_{k}} \dot{q}_{j} \dot{q}_{k}\right) \\
& +2\left(\sum_{j=1}^{h} \frac{\partial x_{\alpha, i}}{\partial q_{j}} \frac{\partial x_{\alpha}, 1}{\partial t} \dot{q}_{j}\right)+\left(\frac{\partial x_{\alpha}, i}{\partial t}\right)^{2}
\end{aligned}
$$

And Eq. III-18 becomes

$$
\begin{aligned}
T=1 / 2 \sum_{\alpha=1}^{n} \sum_{i=1}^{3} m_{\alpha}\left[\left(\sum_{j=1}^{n} \sum_{k=1}^{n} \frac{\partial x_{\alpha}, 1}{\partial q_{j}} \frac{\partial x_{\alpha}, 1}{\partial q_{k}} \dot{q}_{j} \dot{q}_{k}\right)\right. \\
\left.+2\left(\sum_{j=1}^{n} \frac{\partial x_{\alpha}, 1}{\partial q_{j}} \frac{\partial x_{\alpha \cdot i}}{\partial t} \dot{q}_{j}\right)+\left(\frac{\partial x_{\alpha}, l}{\partial t}\right)^{2}\right] \text { (III }
\end{aligned}
$$

If the system is scleronomic, so that time does not appear explicitly in the equations of transformation, then the partial time derivatives vanish, and therefore the kinetic energy is a homogeneous quadratic function of the generalized velocities:

$$
T=\sum_{\alpha} \sum_{i j k}(1 / 2) m_{\alpha} \frac{\partial x_{a} ;}{\partial q_{j}} \frac{\partial x_{a}, i}{\partial q_{k}} \dot{q}_{j} \dot{q}_{k}
$$

Thus, we can write down the kinetic energy

$$
T=1 / 2 \sum_{j, k=1}^{n} m_{j k} \dot{q}_{j} \dot{q}_{k}
$$


And, in general

$$
\frac{\partial T}{\partial q_{k}}=0 \quad k=1,2, \ldots \ldots, n
$$

Potential Energy in Generalized Coordinate System

Therefore from $\mathrm{Eq}$. III-17 and $\mathrm{Eq}$. III-28 we have

$$
\left.\frac{\partial T}{\partial q_{k}}\right|_{0}=0, \quad k=1,2, \ldots \ldots, n
$$

Eq. III-29 means that the potential energy has an extremum at the equilibrium configuration of the system, $q_{k 0}$, where $k=1,2, \ldots, n$. If the configuration is initially at the zero equilibrium position, we choose $q_{k 0}=0$. If, orlginally, $q_{k 0}^{\prime} \neq 0$, we can always use a linear transformation to set $q_{k 0}=0$. Now, if we expand the potential energy in a Taylor series about the equiI librium configuration, we get

$$
\begin{aligned}
u\left(q_{1}, q_{2}, \ldots, q_{n}\right)= & u_{0}+\left.\sum_{k=1}^{n} \frac{\partial U}{\partial q_{k}}\right|_{0} q_{k} \\
& +1 /\left.2 \sum_{j, k=1}^{n} \frac{\partial^{2} u}{\partial q_{j} \partial q_{k}}\right|_{0} q_{j} q_{k}+\ldots
\end{aligned}
$$

In Eq. III-30, the terms linear, in $q_{k}$ vanish automatically in consequence of the equilibrium conditions (Eq. III-29). The first term in Eq. III-30 is the potential energy of the equilibrium position, and by shifting the arbitrary zero of potential to coincide 
with the equilibrium potential, this term may also be made to vanish. Then, if the motion of the generallzed coordinate system is restricted to be small, all the terms in the expansion which contain products of the $q_{k}$ of degree $h l g h e r$ than second may be neglected. This is equivalent to restricting our interest to simple harmonic oscillations, in which case only the terms quadratic in the coordinates are left. Thus,

$$
U=1 / 2 \sum_{j=1}^{n} \sum_{k=1}^{n} f_{j, k} q_{j} a_{k},
$$

where we define

$$
f_{k, j}=f_{j, k}=\left.\frac{\partial^{2} u}{\partial q_{j} \partial q_{k}}\right|_{0}
$$

The motion of the system is specified to take place in the vicinity of the equilibrium configuration, and the potential energy has an extremum when the system is in this configuration. Since the potential energy, $U$, is chosen to be zero at equilibrium, in general $U$ must be greater than or equal to zero. And it is clear that both potential energy and kinetic energy are positive definite quantities, unless the coordinates, in the case of $U$, or the velocities, in the case of $T$, are zero, in which case they vanish. 
Coefficlents of the Energy Equations in Generalized

Coordinate system

We have concluded that the kinetic energy and the potential energy in the generalized coordinate system have the same form:

$$
\begin{aligned}
& T=1 / 2 \sum_{j=1}^{n} \sum_{k=1}^{n} m_{j, k} \dot{q}_{j} \dot{q}_{k} \\
& U=1 / 2 \sum_{j=1}^{n} \sum_{k=1}^{n} f_{j, k} q_{j} q_{k}
\end{aligned}
$$

By comparing Eqs. III-26 and III-27, it is clear that the $m_{j, k}$ are, in general, functions of coordinates $q_{k}$, and they may be expanded in a Taylor series about the equillorium configuration,

$$
m_{j, k}\left(q_{l}, \ldots q_{n}\right)=m_{j k}\left(q_{\ell 0}\right)+\left.\sum_{l} \frac{M j k}{q_{l}}\right|_{0} q_{l}+\ldots \ldots
$$

$(I I I-34)$

The lowest nonvanishing approximation to $T$,' since in Eq. III-33 $T$ is already quadratic in $a_{k} s$, is obtained by dropping all but the first term in Eq. III-34. Thus the coefficients, $m_{j k}$, become constants and we rewrite equation III -27 in the form

$$
T=1 / 2 \sum_{j=1}^{n} \sum_{k=1}^{n} m_{j k} \dot{q}_{j} \dot{q}_{k}
$$


From Eq. III-32, the coefficients $f_{j k}$ are only numbers.. Thus, in Eq. III-33, the $m_{j k}$ and the $f_{j k}$ are $n$ by $n$ arrays of numbers which specify the way in which the motions of the varlous coordinates are coupled.

Equations of Motion in Generallzed Coordinate system

From Eq. III-33, the Lagrangian is given by

$$
L=1 / 2 \sum_{j=1}^{n} \sum_{k=1}^{n}\left(m_{j k} \dot{q}_{j} \dot{q}_{k}-f_{j k} q_{j} q_{k}\right)
$$

But, since $T$ is a function only of the generallzed velocities and $U$ is a function only of the generalized coordinates Lagrange"s equation for the $k^{\text {th }}$ coordinate becomes

$$
\frac{\partial U}{\partial q_{k}}+\frac{d}{d t} \frac{\partial T}{\partial \dot{q}_{k}}=0
$$

The first-order partial differentiations with respect to the coordinate system of $E q$. II -33 are

$$
\begin{aligned}
& \frac{\partial U}{\partial q_{k}}=(1 / 2) \quad \sum_{j=1}^{n} f_{j k} q_{j} \\
& \frac{\partial T}{\partial q_{k}}=(1 / 2) \quad \sum_{j=1}^{n} m_{j k} \dot{q}_{j}
\end{aligned}
$$

The equations of motion then become

$$
\sum_{j=1}^{n} \quad f_{j k} q_{j}+m_{j k} \ddot{q}_{j}=0,
$$


where explicit use has been made of the symmetry property of the $f_{j k}$ and $m_{j k}$ coefficients. Each of the equations II -39 will involve, In general, all of the coordinates $q_{k}$, and it is this set of simultaneous differential equations which must be solved to obtain the motion near the equilibrium.

The equations of motion, III-39, are a set of $n$ second-order linear homogeneous differential equations with constant coefficients. Since we are dealing with an oscillatory system, we expect a solution of the form

$$
q_{j}(t)=a_{j} \exp i(\omega t-\delta)
$$

where a, gives the complex amplitude of the osclilation for each coordinate, and the phase, $\delta$, has been included to give the two arbitrary constants, aj and $\delta$, required by the second-order nature of each of the differential equations. It is understood, of course, that it is the real part of Eq. III-40 which is to correspond to the actual motion. Substitution of the solution, Eq. III-40, into the equations of motion leads to the following equations for the amplitude factors:

$$
\sum_{j=1}^{n}\left(f_{j k}-w^{2} m_{j k}\right) a_{j}=0
$$

This is a set of $n$ linear homogeneous algebraic equations that the $a_{j}$ must satisfy. For a nontrivial solution to exist, the determinant of the coefficients must vanish. 
If $\omega^{a}$ is denoted by $\lambda$, then

$$
\left|f_{j k}-\lambda m_{j k}\right|=0
$$

To be more explicit, this is a determinant of the order $n \times n:$

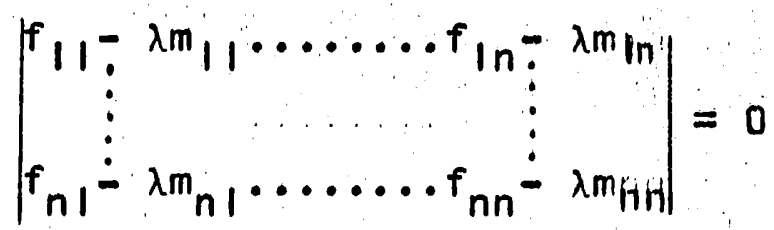

The equation which is represented by this determinant is called the characteristic equation or secular equation of the system, and is an equation of degree $n$ in $\omega^{2}$. The roots of the determinant provide the frequencies for which Eq. III -40 represents a correct solution to the equations of the motion. For each of these values of $w^{2}$, the Eqs. III-4I may be solved for $n-1$ of the ampli,tudes in terms of remaining $a_{j}$. For the case of degeneracy, the problem can be solved by specific algebraic techniques which are omitted in this discussion.

Since the principle of superposition applies for the Eqs. III-4I, the general solution for $q_{j}$ must be written as the superposition of the solutions for each of the $n$ values:

$$
q_{j}(t)=\sum_{r=1}^{n} a_{j r} \exp \left(\omega_{r} t-\delta_{r}\right)=\sum_{r} a_{j r} \cos \left(\omega_{r} t-\delta_{r}\right)
$$

The motion of the coordinate $q_{j}$ is therefore compounded of motions with each of the $n$ values of the frequencles. 
Hence, simplifled coordinate systems are desirable.

B. Mass-Weighted Cartesian Coordinates (MWCC)

and the Principal Axis Transformation

Mass-Weighted Carteslan Coordinates

Suppose the approprlate generalized coordinates were the Cartesian coordinates of the system particles. The klnetlc energy then would contain only the squares of the veloclty components. By introducing generalized coordinates, which are the Cartesian components multiplied by the square root of the particle mass, the kinetic energy can be put in the form

$$
T=1 / 2 \sum_{j=1}^{n} \sum_{k=1}^{n} \delta_{j k} \dot{q}_{j} \dot{q}_{k}
$$

The Kronecker delta, $\delta$, equals unity if $j=k$, and is zero otherwise. By comparing Eqs. III -27 and III -45 , we see that in this coordinate system $m_{j k}=0_{j k}$. This coordinate system is called mass-weighted Carteslan coordinates. And the kinetic energy can be written

$$
T=(1 / 2)\left(\sum_{j=1}^{n} \quad \dot{q}_{j}^{2}\right)
$$

Hency, Eqs. II I -39 become

$$
\ddot{q}_{j}+\sum_{k=1}^{n} f_{j k} q_{j}=0
$$


and $E q$. II I -4 I can be written

$$
\sum_{j=1}^{n}\left(f_{j k} a_{j}-\delta_{j k} w^{2} a_{j}\right)=0
$$

If we replace $w^{3}$ by $\lambda$, Eq. III-48 is of the form

$$
\sum_{j=1}^{n}\left(f_{j k}-\delta_{j k} \lambda\right) a_{j}=0
$$

According to the same argument as in the previous section, Eq. III-43 in this case is written

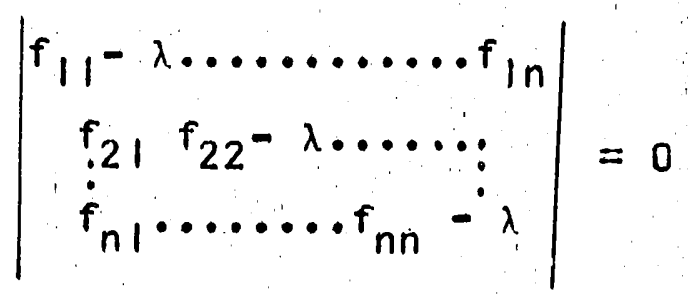

\section{The Principal Axis Transformation}

For a given set of particles, in view of the identification between tensor quantities and the Hermitian matrices, one can find a set of Carteslan axes for which the tensor elements under investigation will be diagonal. This set of axes is called the "princlpal axes". If the initial set of coordinates is not principal axes, one can always transform this initial set to the principal axes by a particular orthogonal transformation, known as the "Principal Axis Transformation".

Let us now return to Eq. III -41 which, in matrix form, can be written

$$
F A=\Lambda M A
$$


The effect of $F$ on $A$ is such as to produce a multiple of the result of $M$ acting on $A$, not merely to produce $\Sigma \lambda$ a. In consequence of the Hermitian property of $F$ and $M$, it will be shown that the elgenvalues, $\lambda$, for which equation III-5I can be satisfled are all real and positive, and the efgenvectors, $a$, which are the column vectors of matrix $A$, are orthogonal. In addition, the matrix of the efgenvectors, $A$, diagonalizes both $M$ and $F$, the former to the unit matrix, E, and the latter to a matrix whose diagonal elements are the elgenvalues, $\lambda$.

If we let $a_{j k}$ represent the jth component of the kth efgenvector, Eq. III-4I can be written as

$$
\sum_{j=1}^{n} F_{i j} a_{j k}=\lambda_{k} \sum_{j=1}^{n} M_{i j} a_{j k}
$$

The complex conjugate of the similar equation for $\lambda_{\ell}$ has the form

$$
\sum_{i=1}^{n} F_{i j} \stackrel{*}{a}_{i \ell}=\stackrel{*}{\lambda}_{\ell} \sum_{i=1}^{n} M_{i j}{\stackrel{*}{a_{i \ell}}}^{\prime}
$$

We multiply Eq. III -53 by $a_{j k}$, sum over $j$, and subtract the resulting equation from the similar product of Eq. III-52 with $a_{i l}^{*}$ summed over 1 . The left-hand side of the difference equation vanishes, leaving only

$$
0=\left(\lambda_{k}-\lambda_{k}^{*}\right) \sum_{i=1}^{n} \sum_{j=1}^{n} M_{i j} a_{j k} a_{i \ell}^{*}
$$


Consider first the special case of Eq. III -54 when $l=k$ :

$$
\left(\lambda_{k}-\lambda_{k}^{*}\right) \quad \sum_{i=1}^{n} \sum_{j=1}^{n} M_{i j} a_{j k} a_{i j k}^{*}=0
$$

The sum over $\mid$ and $j$ will now be shown to be real and positive definite. To verify this statement, separate $a_{j k}$ into its real and imaginary components:

$$
a_{j k}=\left(\alpha_{j k}, \beta_{j k}\right)
$$

The summation can then be written:

$$
\begin{aligned}
\sum_{i=1}^{n} \sum_{j=1}^{n} M_{i j} a_{j k} a_{i k}^{*}= & {\left[\sum_{i=1}^{n} \sum_{j=1}^{n} M_{i j} \alpha_{j k} \alpha_{i k}\right.} \\
& +\sum_{i=1}^{n} \sum_{j=1}^{n} M_{i j} \beta_{j k} \beta_{i k,} \\
& \left.\sum_{i=1}^{n} \sum_{j=1}^{n} M_{i j}\left(\beta_{j k} \alpha_{i k}-\beta_{j k} \alpha_{j k}\right)\right]
\end{aligned}
$$

The imaginary term in Eq. III -57 vanishes in consequence of the symmetry of $M_{i j}$, for an interchange of the dummy indices, $i$ and $j$, changes the sign of the summation. Hence the sum is real. Furthermore, it is seen from the definition of the coefficients $M_{i j}$, Eq. III-34, the summation in Eq. III-55 cannot be zero for real and positive, definite kinetic energy. It follows that

$$
\lambda_{k}=\lambda_{k}^{*}
$$

and eigenvalues $\lambda_{k}$ must be real. 
Since the eigenvalues are real, the ratio of the eigenvector components a jk determined by Eq. III-52 must all be real. Multiply Eq. III -52 by $a_{i k}$ and sum over i:

$$
\sum_{i=1}^{n} \sum_{j=1}^{n} F_{i j} a_{i k} a_{j k}=\lambda_{k} \sum_{i=1}^{n} \sum_{j=1}^{n} M_{i j} a_{i k} a_{j k}
$$

we get an equation which can be solved for $\lambda_{k}$ :

$$
\lambda_{k}=\frac{\Sigma \Sigma F_{i j} a_{i k} a_{j k}}{\Sigma \Sigma M_{i j} a_{i k} a_{j k}}
$$

In Eq. II -60 neither numerator nor denominator can be negative, and the denominator cannot be zero. Hence $\lambda$ is always finlte and positive or zero. Therefore, as $\lambda$ stands for $\omega^{2}$, positive $\lambda$ corresponds to real frequencies of oscillation.

In view of the reality of the eigenvalues and eigenvectors, Eq. III -54 can be written

$$
\left(\lambda_{k}-\lambda_{\ell}\right) \sum_{i=1}^{n} \sum_{j=1}^{n} M_{i j} a_{i \ell} a_{j k}=0
$$

If all the roots of the secular equation are distinct, then Eq. III-6I can hold only if the summation vanishes for $l$ not equal to $k$ :

$$
\sum_{i=1}^{n} \sum_{j=1}^{n} M_{i j} a_{i \ell} a_{j k}=0 \quad l \neq k
$$


If we require that

$$
\sum_{i=1}^{n} \sum_{j=1}^{n} M_{1 j} a_{i l} a_{j k}=1 \text { when } \&=k
$$

Eqs. III -62 and I I -63 can be combined as:

$$
\sum_{i=1}^{n} \sum_{j=1}^{n} M_{i j} a_{i l} a_{j k}=\delta_{\ell k}
$$

In matrix notation, Eq. III-64 can be written as

$$
\tilde{A} M A=E
$$

Eq: III-65 is the orthogonality condition for the matrix $A$ in the configuration space whose metric tensor is $M$.

In a Cartesian space the metric tensor is the unit tensor $E$ and the condition III -65 is reduced to

$$
\tilde{A} A=E
$$

This is the ordinary orthogonality requirement.

In the Eq. III-52, if we define $\lambda_{\ell k}=\lambda_{k}{ }^{\delta} k^{\text {, }}$ the expression can be written

$$
\sum_{j=1}^{n} F_{i j} a_{j k}=\sum_{j=1}^{n} M_{j j} a_{j k} \lambda_{l k},
$$

which becomes in matrix notation:

$$
F A=M A \Lambda \text {. }
$$

Multiplying $\tilde{A}$ from the left, Eq. III-68 takes the form

$$
\tilde{A} F A=\tilde{A} M A \Lambda \text {, }
$$

which, by Eq. III -65 reduces to

$$
\tilde{A} F A=\Lambda \text {. }
$$


Eq. III-69 states that a congruent transformation of $F$ by A changes it into a diagonal matrix whose elements are the eigenvalues $\lambda_{K}$.

The matrix A thus simultaneously diagonalizes both $F$ and $M$. The diagonalization process is interpreted as follows: A is the matrix of a linear transformation from a system of Inclined axes to Cartesian orthogonal axes. At the same time, the new axes are the perpendicular principal axes of $F$, so that the matrix $F$ is diagonal in the transformed coordinate system. Therefore, we can conclude that the entire process of obtaining the fundamental frequencies of small osclilation is a particular type of principal axis transformation.

C. Normal Coordinates and Normal Mode of Vibration

The coordinate system used in the previous sections are not, in general, the separation coordinates of the problem, because not every one of them is simply periodic and each solution is not necessarily representing a fundamental frequency of the particle system under consideration. However, we can obtain such a set of periodic coordinates by a point transformation from the previous set of coordinates $q_{j}$.

We can define a new set of coordinates $\xi_{j}$ related to the original coordinates by the equations

$$
q_{1}=\sum_{j} a_{i j} \xi_{j}
$$


If $q_{j}$ and $\xi_{j}$ are represented as the elements of single-column matrices $Q$ and $\Xi$ respectively, the defining equation, Eq. III-7I, appears as

$$
Q=A \Xi
$$

The potential energy can be written as

$$
U=(1 / 2) \tilde{Q} F Q \text {. }
$$

and the kinetic energy can be expressed as

$$
T=(1 / 2) \tilde{\dot{Q}} M \dot{Q}
$$

Now, since

$$
Q=\tilde{A} \Xi=\tilde{\Xi} \tilde{A}
$$

Eq. III -73 becomes $U=(1 / 2)$ EAFAE

According to Eq. III -70 , the potential energy reduces

simply to

$$
U=(1 / 2) \tilde{E} \Lambda \Xi
$$

Written explicitly, Eq. III-77 appears as

$$
U=(1 / 2) \sum_{k} w_{k}^{a} \xi_{k}^{a}
$$

In the new coordinates, the kinetic energy can be written

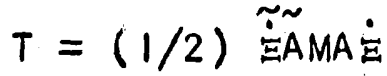

and according to Eq. III-65

$$
T=(1 / 2) \stackrel{\tilde{\Xi}}{\dot{\Xi}}
$$

In terms of the new velocities, the kinetic energy is therefore

$$
T=(1 / 2) \sum_{k} \xi_{k}^{a}
$$

Equations III-79 and III-8I state that in the new coordinates both the potential and kinetic energies are sums 
of squares only, without any cross terms. The Lagrange equations for $\xi_{k}$ are

$$
\ddot{\xi}_{k}+\omega_{k}^{2} \xi_{k}=0
$$

These equations have solutions

$$
\xi_{k}=c_{k} \exp \left(-1 w_{k} t\right)
$$

It is easily seen that each of the new coordinates is a simply perlodic function involving only one of the resonant frequencies. Customarily, $\xi^{\prime}$ 's are called normal coordinates of the system.

Each normal coordinate corresponds to a vibration of the system with only one frequency, and these component oscillations are spoken of as the normal modes of vibration. All of the particles in each mode vibrate with the same frequency and with the same, phase, the relative amplitudes being determined by the matrix elements $a_{i k}$. The complete motion is built up out of the sum of the normal modes weighted with appropriate amplitude and phase factors contained in the $C_{k}$ 's.

\section{Internal Coordinates}

In order to set up and carry out the actual computation in this investigation, it is more convenient to apply the six conditions of no rotation and no translation before the solution of the secular equation. This kind of reduction in the number of original coordinates may be carried out in two ways. One method is to use the six 
conditions to express six of the original coordinates in terms of the remaining $3 N-6$ coordinates. The other method is to introduce a new set of $3 N-6$ coordinates $s_{1}, s_{3}, \ldots$, $S_{3 N-6}$ which are defined by means of the six conditions and $3 N-6$ relations connecting the s's with the originai coordinates. These coordinate systems, which describe the internal configuration of the molecule without regard for its position as a whole in space, are known as internal coordinates.

The set of $3 N-6$ internal coordinates used in this paper is provided by changes in interatomic distances and changes in the angles between chemical bonds. This type of coordinate provides the most physically significant set of coordinates for use in describing the potential energy of the molecule. But the kinetic energy is more easily set up in terms of Cartesian displacement coordinates of the atoms. A relation between the two types is therefore demanded. Each of the 3N-6 internal coordinates can be considered as the linear combination of the $3 \mathrm{~N}$ Cartesian displacement coordinates. If $\mathrm{S}_{t}$ represents one of the $3 N-6$ internal coordinates and $\xi_{i}$ one of $3 \mathrm{~N}$ Cartesian displacement coordinates, then

$$
s_{t}=\sum_{i=1}^{3 N} B_{t i} \xi_{i} \quad t=1,2, \ldots, 3 N-6
$$

where the coefficients $B_{t i}$ are constants determined by the geometry of the molecule. If we define a vector $\hat{\rho}_{\alpha}$ 
whose components along the three axis directions are the Cartesian displacement coordinates $\xi_{j}, \xi_{j}, \xi_{k}$ for atom $a$, and a vector $\hat{s}_{t \alpha}$ whose components are $\hat{B}_{t j}, \hat{B}_{t j}, \hat{B}_{t k}$ associated with atom $\alpha$, then Eqs. III-84 can be written $a^{27}$

$$
s_{t}=\sum_{\alpha=1}^{N} \hat{s}_{t \alpha} \cdot \hat{p}_{\alpha}
$$

Physically, the direction of $\hat{s}_{t \alpha}$ is the direction in which a given displacement of atom $\alpha$ will produce the greatest increase of $s_{t}$. The modulus of $s_{t \alpha}$ is equal to the increase in $s_{t}$ produced by unit displacement of the atom in this most effective direction.

G Matrix and the Secular Equations in Terms of Internal Coordinates

In terms of mass-weighted Cartesian coordinates the kinetic energy is given by Eq. III-45. In matrix notation it is

$$
T=(1 / 2) \tilde{\dot{Q}} \dot{Q}
$$

In terms of momentum $P$,

$$
\text { where } p_{i}=\frac{\partial T}{\partial \dot{q}_{1}}=\dot{q}_{i}
$$

Eq. III -86 is written

$$
T=(1 / 2) \tilde{P} P
$$

We let the transformation from the mass-weighted Cartesian displacement coordinates to internal coordinates be

$$
S=D Q
$$


where $D_{t j}=B_{t j} m_{j}$, $m$ being the mass of the particle involved. If we consider $T$, the kinetic energy, as a function of velocities in the internal coordinates system, then

$$
p_{i}=\frac{\partial T}{\partial \dot{q}_{1}}=\sum_{t} \frac{\partial T}{\partial \dot{S}_{t}} \frac{\partial \dot{S}_{t}}{\partial \dot{q}_{1}}
$$

But

$$
\frac{\partial T}{\partial \dot{S}_{t}}=P_{t}
$$

and

$$
\frac{\partial \dot{s}_{t}}{\partial \dot{q}_{i}}=\frac{\partial s_{t}}{\partial q_{i}}=D_{t i}
$$

so Eq. III-90 can be written as

$$
p_{i}=\sum_{t} P_{t} D_{t i}
$$

and in matrix notation

$$
\tilde{p}=\tilde{P} D
$$

According to Eq. III -88 , we get

$$
T=(1 / 2) \tilde{P} D \tilde{D P}
$$

where

$$
\begin{aligned}
(D \tilde{D})_{t t^{\prime}} & =\sum_{i} D_{t i} \tilde{D}_{i t^{\prime}} \\
& =\sum_{i} D_{t i} D_{t^{\prime} i} \\
& =\sum_{i} M_{i}^{-1} B_{t i} B_{t^{\prime} i}^{*}
\end{aligned}
$$

In Euclidean space; we can define ${ }^{28}$

$$
G_{t t^{\prime}}=\sum_{i=1}^{3 N} \quad \frac{1}{m_{i}} B_{t i} B_{t^{\prime}}
$$


where $t, t^{\prime}=1,2, \ldots \ldots, 3 N-6$.

In terms of vectors, $G_{t t^{\prime}}$ is given by

$$
G_{t t^{\prime}}=\sum_{\alpha=1}^{N} \mu_{\alpha} \hat{S}_{t \alpha} \cdot \hat{S}_{t^{\prime} \alpha}
$$

where $\mu_{\alpha}=\frac{1}{m_{\alpha}}$ is the reciprocal of the mass of atom $\alpha$.

The kinetic energy now can be written as

$$
T=(1 / 2) \tilde{P} \text { GP. }
$$

If the determinant of $G$ does not vanish and the inverse of $G$ exists, then

$$
\dot{S}_{t}=\frac{\partial T}{\partial P_{t}}
$$

and $\dot{s}=$ GP.

Equation III -88 can be' written as 29

$$
T=(1 / 2) \tilde{\dot{S}} G^{-1} \dot{S} \text {. }
$$

Similarly, we can express the potentlal energy in terms of the same internal coordinates

$$
V=(1 / 2) \cdot \tilde{S} F S
$$

Then the problem of small vibration leads to the secular equation

$$
\left|F-G^{-1} \lambda\right|=0
$$

By applying matrix algebra to the above equation, Eq.

II I I03 becomes

$$
|G F-E \lambda|=0^{\prime}
$$

where $F$ is the matrix of force constants whose elements can be estimated directly in terms of bond distances and angles between. involved atoms. 
Since both $F$ and $G$ matrices are symmetrical, rows

and columns in Eq. III-104 can be interchanged to yleld the following form

$$
|F G-E \lambda|=0
$$

where $\lambda=4 \pi^{2} c^{2} \omega_{i}^{2}$.

Eq. III-105 is the secular equation used in this investigation to calculate the vibrational frequencies. 
Chapter IV - Formulations and Calculations

1. Molecular Fragmentation

A. General Description

B. Molecular Fragmentation Method

C. Application of Molecular Fragmentation to the Decarboxylation of Malonic Acid

2. The "G" Matrix

81

A. The Three-Center Fragment

Valence Bond Stretching Coordinates

Valence Angle Bending Coordinate

The Elements of "G" Matrix for Three-Center

Fragment

B. The Four-Center Fragment

Valence Bond Stretching Coordinates

Valence Angle Bending Coordinates

The Elements of "G" Matrix for Four-Center

Fragment

3. Calculations and Results 94 
1. Molecular Fragmentation Method

\section{A. General Description}

According to Eq. II -55 we see that the transition state formulation for the ratio of rate constants for unimolecular reactions differing in isotoplc constitution is

$$
\frac{k^{\prime}}{k}=\frac{\nu_{x}^{\prime}}{\nu_{x}} \prod_{i}^{3 N-6} \frac{U_{i} \sinh \left(\frac{U_{i}^{\prime}}{2}\right)}{U_{i} \sinh \left(\frac{U_{i}}{2}\right)} \quad \prod_{i}^{3 N-7} \frac{U_{i}^{*} \sinh \left(\frac{U_{i}^{*}}{2}\right)}{U_{i}^{*} \sinh \left(\frac{U_{i}^{*}}{2}\right)}
$$

where primed and unprimed quantities refer to the two isotopic species and $\neq$ refers to the transition-state structure, $v_{x}$ is the frequency (or average frequency) of motion in the reaction coordinate, and $U_{i}=h c \omega_{1} / K T$ where $\omega_{i}$ is a vibrational frequency (or a fundamental frequency of vibration).

The vibrational frequencies of the activated complex are often calculated by WIIson's F, G, matrix methods 29. The $G$ matrix is specified by assuming a physically reasonable structure for the activated complex. The diagonal elements of the $F$ matrix can be estimated from the assumed structure by analogy to stable molecules; the off-diagonal elements might be set equal to zero or adjusted to conform to assumption about the nature of the reaction coordinate 30,31 . The vibrational frequencies are obtained from the roots of Eq. III-105. The reactant frequencles may be obtained by a similar calculation or from spectroscopic data. However, the continued product 
Involving reactant frequencies in Eq. IV-I reduces to unity for an intramolecular isotope effect, where both reactant species are identical. In this investigation a speciflc example chosen is the decarboxylation of end-labelled malonic acid

$$
\int_{C_{C}^{12} \mathrm{OOH}}^{\stackrel{\mathrm{k}_{3}}{\longrightarrow} \mathrm{C}^{12} \mathrm{H}_{3} \mathrm{C}^{12} \mathrm{OOH}+\mathrm{C}^{13} \mathrm{O}_{2}}
$$

where $k_{4} / k_{3}$ is a measure of a carbon-12/carbon-13 intramolecular isotope effect. Since the rate-constant ratio depends only on the vibrational frequencies of the activated complexes for this type of isotope effect, approximations and assumptions about the transition state that simplify the secular equations are particularly useful in these cases.

B. Molecular Fragmentation Method

If the assumption is made that the activated complex can be treated as vibrationally decoupled fragments, its secular equation is considerably simplified. Uncoupled, freely rotating models for the activated complex have been used in a number of absolute-rate theory calculations of rate constants 32 . These applications have been 
quite properly limited to dissociations with abnormally large pre-exponential factors (and the reverse recombinations), since the change of several internal modes of vibration in the reactant to free rotations in the activated complex must be associated with abnormally large entropies of activation. However, in calculations of ratios of rate constants for isotopic molecules, the decoupled fragment approximation has a broader range of validity. If the activated complex has a "loose" structure, with weakly coupled fragments, motions between fragments involve vibrations of much lower frequency than motions within fragments. The approximate separation of high and low frequencies 29 can then be applied to factor the secular equation into intra- and interfragment parts. The low-frequency, inter-fragment vibrations, although very important in calculations of an individual rate constant, have little effect on the continued product in Eq. IV-1, since $\left[(U / 2)^{-1} \sinh (U / 2)\right]$ approaches unity for small $U$. The rows and columns of the secular equation that correspond to inter-fragment motions can therefore be deleted, and the approximate frequencies of internal vibrations of the fragments, obtained from the reduced secular equation are used in Eq. IV -1 .

For a reaction involving rupture (or formation) of one bond, $A B=A+B$, the activated complex is treated as two fragments, and motion in the reaction coordinate is 
change in the distance between the centers of mass of the two fragments. The theoretical expression for an intramolecular isotope effect for this case becomes:

$$
\begin{aligned}
& k^{\prime} k=\left[\frac{M_{A B}^{\prime}}{M_{A B}}\right]^{3 / 2}\left[\frac{I^{\prime} B}{I_{A B}}\right]^{1 / 2}\left[\frac{A^{\prime} B^{\prime} C^{\prime}}{A B^{\prime} C}\right]_{A}^{1 / 2}\left[\frac{A^{\prime} B^{\prime} C^{\prime}}{A^{\prime} B^{\prime} C}\right]_{B}^{1 / 2} \\
& \prod_{i}^{3 n-6} \frac{\sinh \left(U_{A i / 2}^{*}\right)}{\sinh \left(U_{A i / 2}^{\neq 1}\right)} \prod_{i}^{3 n-6} \frac{\sinh \left(U_{B i / 2}^{*}\right)}{\sinh \left(U_{B i}^{* \prime} / 2\right)}
\end{aligned}
$$

Eq. IV-3 is the ratio of the total partition function of the activated complexes with $3 n-6$ degrees of vibrational freedom for each fragment, three rotational degrees of freedom for each fragment, and three translational degrees of freedom for each fragment subject to the constraint. that the distance between the centers of gravity of the two fragments is constant.

Applying the product rule 5 to the complete system yields

$$
\begin{aligned}
& {\left[\frac{\mu_{A B}}{\mu_{A B}^{\prime}}\right]^{1 / 2}\left[\frac{M_{A B}}{M_{A B}^{\prime}}\right]^{3 / 2}\left[\frac{I_{A B}}{I_{A B}^{\prime}}\right]\left[\frac{A B C^{\prime}}{A^{\prime} B^{\prime} C^{\top}}\right]_{A}^{1 / 2}\left[\frac{A B C}{A^{\prime} B^{\prime} C^{\top}}\right]_{B}^{1 / 2}} \\
& \begin{array}{l}
3 n-6 \\
i
\end{array} \frac{U_{A}^{*} i}{U_{A i}^{*}} \quad \prod_{i}^{3 n-6} \quad \frac{U_{B i}^{*}}{U_{B i}^{*}}=1
\end{aligned}
$$

where $\mu_{A B}=\frac{M_{A} \times M_{B}}{M_{A}+M_{B}}$, the reduced mass of two fragments. 
Alternatively, one can apply the product rule to each fragment independently:

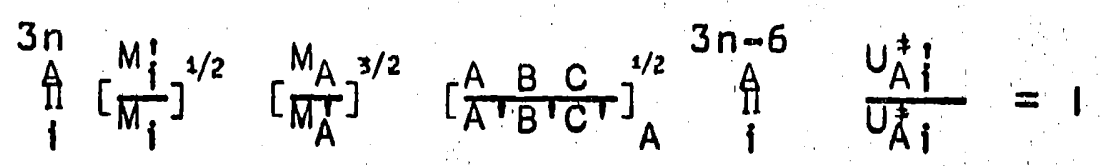

and

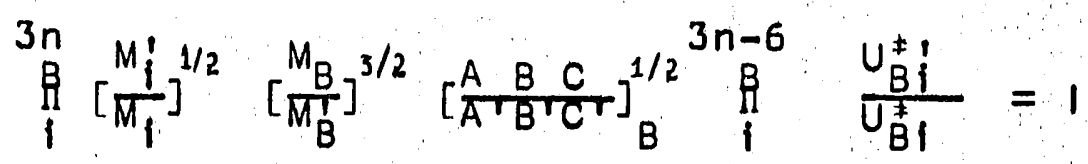

Consequently, Eq. IV-4 becomes:

$$
k^{\prime} / k=\left(\mu_{A B} / \mu_{A B}\right)^{1 / 2} \prod_{i}^{3 n-6} \frac{U_{A}^{*}\left\{\sinh \left(U_{A i}^{*} / 2\right)\right.}{\left.U_{A i}^{*} \sinh \left(U_{A\}}^{*}\right\} / 2\right)} \prod_{i}^{3 n-6} \frac{U_{B i}^{*} \sinh \left(U_{B i}^{*} / 2\right)}{U_{B i}^{*} \sinh \left(U_{B i}^{*} / 2\right)}
$$

The reaction coordinate in this case is the distance between the centers of gravity of the two fragments, $A$ and $B$. Since the average relative velocity 33 of two independent systems is inversly proportional to the square root of their mechanical reduced mass,

$$
\text { TIF }=\nu_{x}^{\prime} / \nu_{X}=\left(\mu_{A B} / \mu_{A B}^{\prime}\right)^{1 / 2}
$$

The extension to intermolecular isotope effects can be made by including a factor for the ratio of total partition functions for reactants: 


$$
\begin{aligned}
& k^{1} / k=\left(\mu_{A B} / \mu_{A B}^{\prime}\right) \prod_{i}^{3 n-6} \frac{U_{R i} \sinh \left(U_{R_{i}} / 2\right)^{3 n-6}}{U_{R_{1}}^{\prime} \sinh \left(U_{R i} / 2\right)} \prod_{i}^{U_{A}^{*} \sinh \left(U_{A i}^{*} / 2\right)} \\
& x \overbrace{i}^{3 n-6} \frac{U_{B i}^{*} \sinh \left(U_{B i}^{\neq} / 2\right)}{U_{B i}^{\neq} \sinh \left(U_{B i}^{\neq} i / 2\right)}
\end{aligned}
$$

However, the fragmentation method is not a good approximation for the reactant molecule, and the reactant frequencies in Eq. IV-g are the conventional ones calculated for the reactant molecule as a whole.

The simplification provided by this method can be easily seen from the comparison of Eqs. I V-3 and IV-7. The calculation of vibrational frequencies for the activated complex is split into a vibrational analysis of each of the fragments. The resulting reduction in the order of the secular equation permits one to treat much more complicated systems than is possible with more rigorous methods.

Reactions involving simultaneous rupture or formation of two or more bonds can also be treated by this type of model in terms of three or more uncoupled fragments. General formulations of $\nu_{x}^{\prime} / \nu_{x}$ for three-centered models which have appeared in the literature 23,24 are applicable in these cases. However, the reaction coordinate can be treated only in terms of inter-fragment motions; the reaction coordinate as a linear combination of internal displacements, within as well as between fragments, 
cannot be incorporated in this approach.

The molecular fragmentation method, which assumes a "loose" activated complex, cannot be applied to reactions that do not involve bond rupture or formation in the rate determining step. In addition, of course, assumption of the validity of quasi-equilibrium theory is implicit in the general expression of Eq. IV -1 .

Despite the limitations mentioned above, the molecular-fragment models have a wide range of application. This approach should be particularly useful and valuable in dealing with large, complicated structures, because it provides simple, pictorial representations for various reaction mechanisms and greatly simplifies the calculation of activated-complex vibrational frequencies. Thus the method could be used, as the next approximation beyond noting the presence or absence of kinetic lsotope effects, in distingulshing between alternate mechanisms, such as concerted or step-wise rupture of two bonds. It could also be used to estimate substituent effects in a serles of analogous isotopic reactions.

C. Application of Molecular Fragmentation Method to the Decarboxylation of Malonic Acid

The decarboxylation of dibasic carboxylic acids is perhaps the type of reaction whose carbon isotope effect has been most thoroughly studied by different research workers. The compound $\mathrm{CH}_{2}(\mathrm{COOH})_{2}$ has two differen't 
isotopic isomers containing one heavy carbon atom. The one which contains the heavy atom in the methylene group could expel carbon dioxide in two identical ways, the molecule belng lootopleally oymmetrie. The unoymmetric isomer could react in two different ways, the heavy carbon being contalned elther in the carbon dioxide or in the acetic acid.

The reaction scheme of a sample containing both isomers of mono-heavy malonic acid together with the ordinary compound is illustrated below for the case of $c^{13}$ (the mass number of carbon is assumed to be 12 if not Indicated):

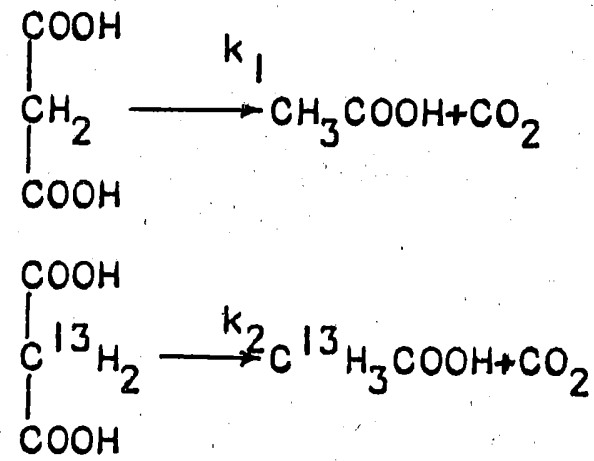

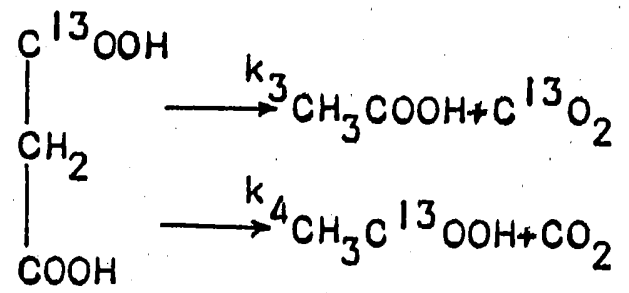

Eq. IV-12 presents the problem of interest in this work. The malonic acid, with eleven atoms, has twenty-six normal modes of vibration. Theoretically, even if the complete vibrational analysis could be carried out, so many parameters would be involved that a meaningful 
interpretation would be virtually impossible. Therefore, for the purpose of actual computation and analysis, a simplified model for the transition-state is desirable. If one assumes that duming the cours of reaction one carbon-carbon bond was very weak in the transition state, then this structure could be treated as two independent fragments.

A further simplification is the omission of the hydrogen atoms from the transition-state models. This is an application of the "cut-off" approximation of Stern and Wolfsberg. 34 Since no hydrogen is bonded directly to the carbon atoms which are isotopically substituted in the intramolecular pair of reactions, the characteristic group frequencies for hydrogen stretching and bending should be nearly identical for $k_{3}$ and $k_{4}$. However, the proton masses are included in calculating the fragment reduced-mass ratio for $\nu_{x}^{1} / \nu_{x}$.

With these "fragmentation" and "cut-off" approximations, the eleven-center activated complex for malonic acid decarboxylation is treated as one four-center fragment and one three-center fragment (as shown in Fig. IV-I), and the 26 th order secular equation is reduced to one 6th-order and one 3rd-order equation. The detailed step-wise mathematical derivation of the $G$ matrices is given in the following section. 

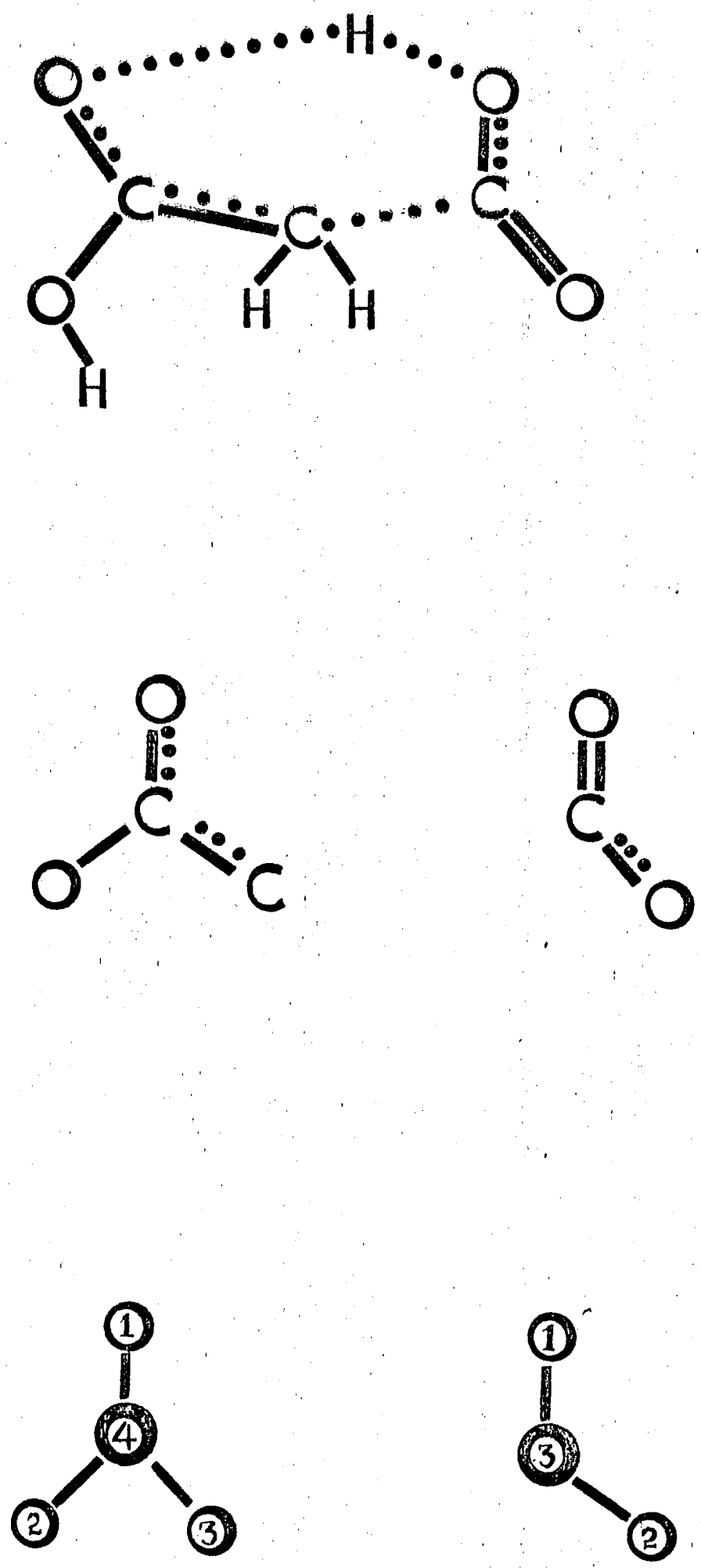

Fig. IV - I 
2. The G Matrix

According to the discussion in Chapter III, the set of internal coordinates chosen in this case are bond stretchings and valence angle bendings. In the case of 3-center model, the three internal coordinates $(3 \times 3-6)$ are two valence bond stretchings and one valence angle bending. In the 4-center case, the six internal coordinates $(3 \times 4-6)$ are three valence bond stretchings and three valence angle bendings.

The notations used here are the same as in Chapter II and are shown in the corresponding figures.

A. The Three-Center Fragment

A non-linear triatomic fragment should possess $3 \times 3-6=3$ degrees of freedom. Therefore three coordinates, two bond stretchings and one valence angle bending, are chosen according to the internal coordinate system.

Valence Bond Stretching Coordinates

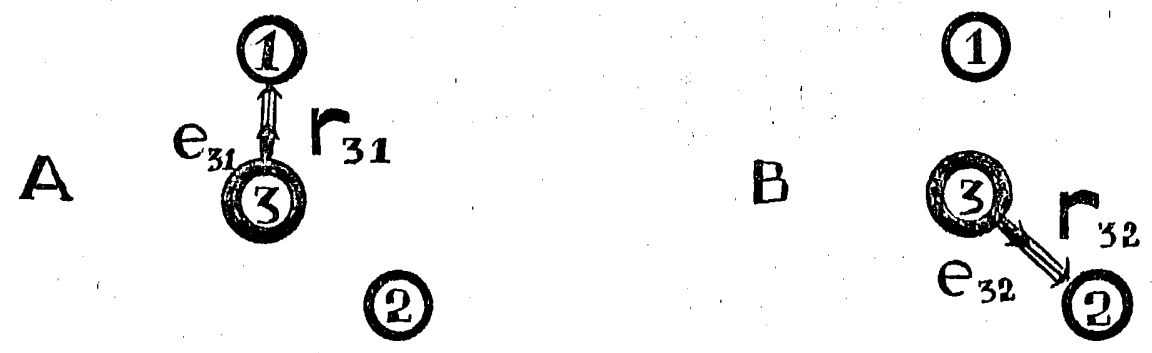

Fig. IV-2 The valence bond stretching coordinates.

A. The valence bond stretching between atom 3 and atom 1 .

B. The valence bond stretching between atom 3 and atom 2 . 
The bond stretching coordinates in, the three-center fragment $S_{1}$ and $S_{2}$ are chosen such that $S_{1}$ describes the stretching between atom 3 and atom 1 and $s_{2}$ describes the otretehing between atom 3 matom 2 . In rigur IV-2 $\hat{r}^{\prime} s$ are the direction bond distance and $\hat{e}^{\prime} \mathbf{s}$ are the unit vectors defined as the maximum bond stretchings along the bond distances accordingly. Then the vectors $\hat{s}_{t \alpha}$ can be easily formulated as

$$
\begin{aligned}
\hat{s}_{t \alpha}: t=1 ; \quad \alpha & =1,2,3 \\
\hat{s}_{11} & =\hat{e}_{31} \\
\hat{s}_{12} & =0 \\
\hat{s}_{13} & =-e_{31} \\
t=2 ; \quad \alpha & =1,2,3 \\
\hat{s}_{21} & =0 \\
\hat{s}_{22} & =e_{32} \\
\hat{s}_{23} & =-e_{32}
\end{aligned}
$$

Valence Angle Bending Coordinate

The valence angle bending coordinate $S_{3}$ is defined according to Eq. III-85:

$$
s_{3}=\Delta \varphi=\sum_{\alpha=1}^{3} \hat{s}_{t \alpha} \cdot \hat{\rho}_{\alpha}
$$


The maximum displacement of $\varphi$ is shown in figure IV -3 .

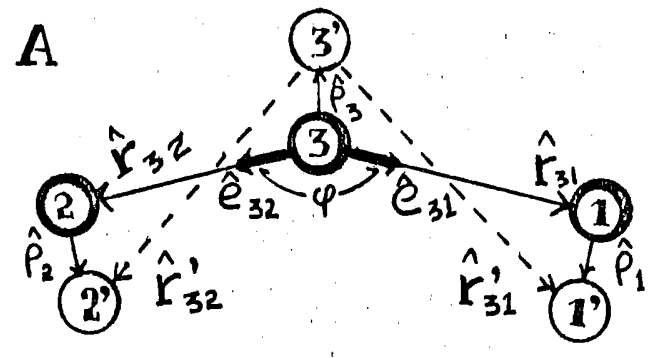

B

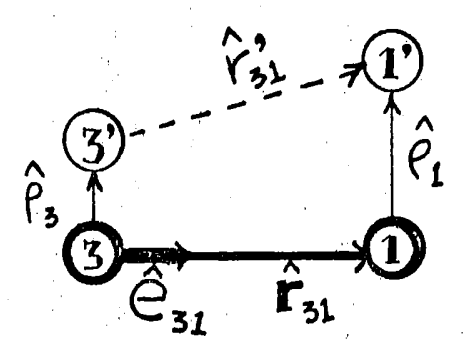

Fig. IV-3 The maximum displacement of $\varphi$.

A. The diagram of the whole system.

B. The relation between atom 3 and atom 1.

From figure IV-3 we can write down the following relations immediately.

$$
\hat{r}_{3 \alpha}^{\prime}=\hat{r}_{3 \alpha}+\hat{p}_{\alpha}-\hat{\rho}_{3} \text { where } \alpha=1,2
$$

then

$$
\hat{r}_{3 \alpha}^{\prime}-\hat{r}_{3 \alpha}=\hat{\rho}_{\alpha}-\hat{\rho}_{3}=\Delta \hat{r}_{3 \alpha}
$$

If we take the inner product of $\hat{r}_{3}$, we get

$$
\hat{r}_{3 \alpha}^{\prime} \cdot \hat{r}_{3 \alpha}^{\prime}=\left(\hat{r}_{3 \alpha}+\hat{\rho}_{\alpha}-\hat{p}_{3}\right) \cdot\left(\hat{r}_{3 \alpha}+\hat{p}_{\alpha}-\hat{p}_{3}\right)
$$

and

$$
\left(\hat{r}_{3 \alpha}^{\prime}\right)^{2}=\left(\hat{r}_{3 \alpha}\right)^{2}+2 \hat{r}_{3 \alpha}\left(\hat{\rho}_{\alpha}-\hat{\rho}_{3}\right)+\left(\hat{\rho}_{\alpha}-\hat{\rho}_{3}\right)^{2}(I V-17)
$$

The third term in the right-hand side of Eq. IV-I7 is the square of the difference between two displacement vectors which is comparatively small and can be neglected. Then Eq. IV-17, according to the basic definition of unit vector, can be expressed as

$$
\hat{e}_{3 \alpha} \cdot\left(\hat{\rho}_{\alpha}-\hat{\rho}_{3}\right)=\Delta\left(r_{3 \alpha}\right)^{2} / 2 r_{3 \alpha}
$$


84

But from Eq. IV -16, we know that

$$
\Delta \hat{r}_{3 \alpha}=\hat{p}_{\alpha}-\hat{p}_{3}
$$

and

$$
\begin{aligned}
& \hat{e}_{3 \alpha} \cdot \Delta \hat{r}_{3 \alpha}=\Delta r_{3 \alpha} \\
\therefore \quad & \Delta r_{3 \alpha}=\hat{e}_{3 \alpha} \cdot\left(\hat{p}_{\alpha}-\hat{\rho}_{3}\right)=\Delta\left(r_{3 \alpha}\right)^{2} / 2 / r_{3 \alpha}
\end{aligned}
$$

Now, look at figure IV -3A we can say that

$$
\cos \varphi=\hat{e}_{31} \cdot \hat{e}_{32}
$$

By differentiating Eq. IV -20, we get

$$
d(\cos \varphi)=-\sin \varphi d \varphi=\hat{e}_{31} \cdot d \hat{e}_{32}+\hat{e}_{32} \cdot d \hat{e}_{31}(I V-2 I)
$$

According to the definition of unit vector, the first differentiation of $\hat{e}_{3 \alpha}=\hat{r}_{3 \alpha} / r_{3 \alpha}$ is

$$
d \hat{e}_{3 \alpha}=\left(r_{3 \alpha} d \hat{r}_{3 \alpha}-\hat{r}_{3 \alpha} d r_{3 \alpha}\right) /\left(r_{3 \alpha}\right)^{2}
$$

Then

$$
\begin{aligned}
\hat{e}_{31} \cdot d \hat{e}_{32} & =\left(r_{32}\right)^{-1}\left(\hat{\rho}_{2}-\hat{\rho}_{3}\right) \cdot \hat{e}_{31} \\
& -\left(r_{32}\right)^{-1}(\cos \varphi) \hat{e}_{32} \cdot\left(\hat{\rho}_{2}-\hat{\rho}_{3}\right) \\
\hat{e}_{32} \cdot d \hat{e}_{31} & =\left(r_{31}\right)^{-1}\left(\hat{\rho}_{1}-\hat{\rho}_{3}\right) \cdot e_{32} \\
& -\left(r_{31}\right)^{-1}(\cos \varphi) \hat{e}_{31} \cdot\left(\hat{\rho}_{1}-\hat{\rho}_{3}\right)
\end{aligned}
$$

Substitute Eq. IV -23 and IV -24 into Eq. IV -21, we have

$$
\begin{aligned}
-\sin \varphi d \varphi & =\left(r_{31}\right)^{-1}\left(\hat{e}_{32}-\cos \varphi \hat{e}_{31}\right) \cdot \hat{\rho}_{1} \\
& +\left(r_{32}\right)^{-1}\left(\hat{e}_{31}-\cos \varphi \hat{e}_{32}\right) \cdot \hat{p}_{2} \\
& +\left[\left(\cos \varphi \hat{e}_{32}-\hat{e}_{31}\right)\left(r_{32}\right)^{-1}\right. \\
& \left.+\left(\cos \varphi \hat{e}_{31}-\hat{e}_{32}\right)\left(r_{31}\right)^{-1}\right] \cdot \hat{p}_{3}
\end{aligned}
$$


then

$$
\begin{aligned}
d \varphi & =\left[\left(\cos \varphi \hat{e}_{31}-\hat{e}_{32}\right)\left(r_{31} \sin \varphi\right)^{-1}\right] \cdot \hat{p}_{1} \\
+ & {\left[\left(\cos \varphi \hat{e}_{32}-\hat{e}_{31}\right)\left(r_{32} \sin \varphi\right)^{-1}\right] \cdot \hat{p}_{2} } \\
+ & {\left[\left[\left(r_{31}-r_{32} \cos \varphi\right) \hat{e}_{31}+\left(r_{32}-r_{31} \cos \varphi\right) \hat{e}_{32}\right]\right.} \\
& \left.\left(r_{3 i} r_{32} \sin \varphi\right)^{-1}\right\} \cdot \hat{\rho}_{3}
\end{aligned}
$$

By comparing Eqs. IV -25 and IV-15, we conclude that

$$
\begin{aligned}
& \hat{s}_{t \alpha}: \quad t=3, \quad \alpha=1,2,3 \\
& \hat{S}_{31}=\left(r_{31} \sin \varphi\right)^{-1}\left(\cos \varphi \hat{e}_{31}-\hat{e}_{32}\right) \\
& \hat{S}_{32}=\left(r_{32} \sin \varphi\right)^{-1}\left(\cos \varphi \hat{e}_{32}-\hat{e}_{31}\right) \\
& \hat{S}_{33}=\left(r_{31} r_{32} \sin \varphi\right)^{-1} \\
& \times\left[\left(r_{31}-r_{32} \cos \varphi\right) \hat{e}_{31}+\left(r_{32}-r_{31} \cos \varphi\right) \hat{e}_{32}\right]
\end{aligned}
$$

\section{The Elements of "G" Matrix for Three-Center Fragment}

The "G" matrix elements now can be formulated according to Eq. III -97

$$
g_{t t^{\prime}}=\sum_{\alpha=1}^{3} \mu_{\alpha} \hat{s}_{t \alpha^{\prime}} \cdot \hat{s}_{t_{\alpha}^{\prime}}
$$

where

$$
\mu_{\alpha}=\left(m_{\alpha}\right)^{-1} ; t, t^{\prime}=1,2,3
$$

Eq. IV-27, in matrix notation, can be written as

$$
G=\left(\begin{array}{lll}
g_{11} & g_{12} & g_{13} \\
g_{21} & g_{22} & g_{23} \\
g_{31} & g_{32} & g_{33}
\end{array}\right)=\left(\begin{array}{l}
\mu_{1} \\
\mu_{2} \\
\mu_{3}
\end{array}\right) \cdot\left(\begin{array}{lll}
\hat{s}_{11} & \hat{s}_{12} & \hat{s}_{13} \\
\hat{s}_{21} & \hat{s}_{22} & \hat{s}_{23} \\
\hat{s}_{31} & \hat{s}_{32} & \hat{s}_{33}
\end{array}\right) \cdot\left(\begin{array}{lll}
\hat{s}_{11} & \hat{s}_{21} & \hat{s}_{31} \\
\hat{s}_{12} & \hat{s}_{22} & \hat{s}_{32} \\
\hat{s}_{13} & \hat{s}_{23} & \hat{s}_{33}
\end{array}\right)
$$

$(I V-28)$ 
By substituting Eqs. IV-13, IV-14, and IV-26 into Eq. IV-28, the "G" matrix elements for the three-center fragment are:

$$
\begin{aligned}
g_{11} & =\mu_{1}+\mu_{3} \\
g_{12} & =g_{21}=\mu_{3} \cos \varphi \\
g_{13} & =g_{31}=-\left(r_{32}\right)^{-1}\left(\mu_{3} \sin \varphi\right) \\
g_{22} & =\mu_{2}+\mu_{3} \\
g_{23} & =g_{32}=-\left(r_{31}\right)^{-1}\left(\mu_{3} \sin \varphi\right) \\
g_{33} & =\left(r_{31}\right)^{-2}\left(\mu_{1}\right)+\left(r_{32}\right)^{-2}\left(\mu_{2}\right) \\
& +\left[\left(r_{31}\right)^{-2}+\left(r_{32}\right)^{-2}-(2 \cos \varphi)\left(r_{31} r_{32}\right)^{-2}\right] \mu_{3}
\end{aligned}
$$

\section{B. The Four-Center Fragment}

This fragment is composed by four atoms, three valence bonds, and three valence angles. Hence, we would have $3 \times 4-6=6$ degrees of freedom. The six internal coordinates are three valence bond stretchings and three valence angle bendings ( $F$ ig. I $V-4$ )

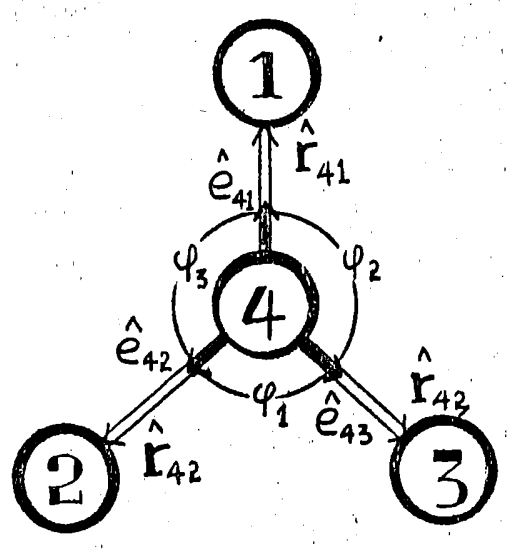

FIg. IV -4 . The four-center fragment 
Valence Bond Stretching Coordinates

Based on the same assumptions made in the previous section, the three valence bond stretching coordinate $S_{1}, S_{2}$, and $s_{3}$ are shown in Fig. IV -5 .
A

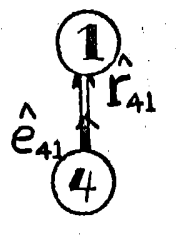
B (1)
(1)
(3)

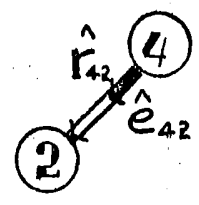
(3)
(2)

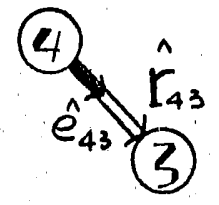

Fig. IV-5 The valence bond stretching coordinates

A. The valence bond stretching between atom 4 and atom 1 .

B. The valence bond stretching between atom 4 and atom 2 .

c. The valence bond stretching between atom 4 and atom 3 .

Then, the vectors $\hat{s}_{t \alpha}$ can be formulated as follows:

$$
\begin{aligned}
\hat{s}_{t \alpha}: t & =1 ; \quad a=1,2,3,4 . \\
\hat{s}_{11} & =\hat{e}_{41} \\
\hat{s}_{12} & =0 \\
\hat{s}_{13} & =0 \\
\hat{s}_{14} & =-\hat{e}_{41} \\
t & =2 ; \quad \alpha=1,2,3,4 . \\
\hat{s}_{21} & =0 \\
\hat{s}_{22} & =\hat{e}_{42} \\
\hat{s}_{23} & =0 \\
\hat{s}_{24} & =-\hat{e}_{42}
\end{aligned}
$$




$$
\begin{aligned}
t=3 ; \quad \alpha & =1,2,3,4 . \\
\hat{s}_{31} & =0 \\
\hat{s}_{32} & =0 \\
\hat{s}_{33} & =\hat{e}_{43} \\
\hat{s}_{34} & =-\hat{e}_{43}
\end{aligned}
$$

\section{Valence Angle Bending Coordinates}

The valence angle bending coordinates of the four center fragment are defined according to Eq. III-85.

$$
s_{t}=\sum_{\alpha=1}^{4} \hat{s}_{t \alpha} \hat{p}_{\alpha} \quad t=4,5,6 .
$$

The maximum displacements of three $\varphi^{\prime} s$ are shown in Fig. IV -6 .
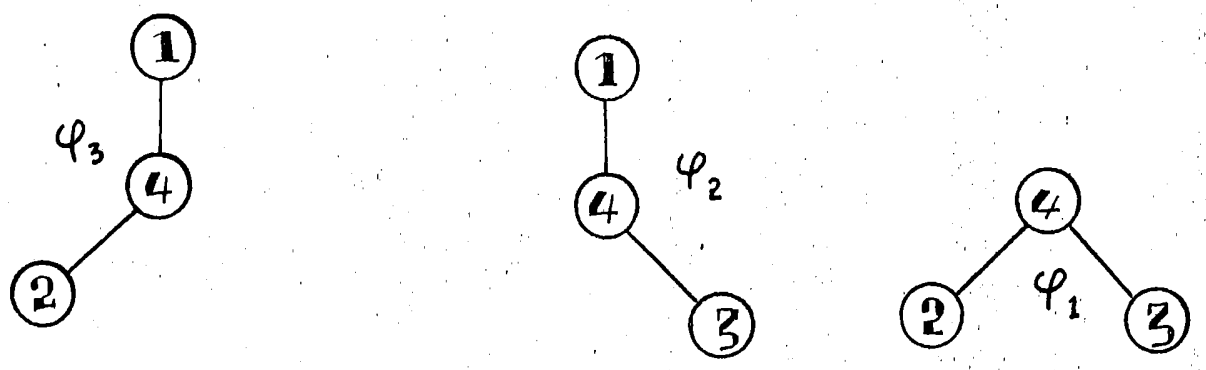

Fig. IV-6. The maximum displacements of $\varphi^{\prime} s$.

$$
\begin{aligned}
& \text { A. } s_{4}=\Delta \varphi_{3} \\
& \text { B. } s_{5}=\Delta \varphi_{1} \\
& \text { c. } s_{6}=\Delta \varphi_{2}
\end{aligned}
$$


From Fig. IV -5 , the unit vectors are defined as

$$
\hat{e}_{4 \alpha}=\hat{r}_{4 \alpha} / r_{4 \alpha} \quad \alpha=1,2,3
$$

The first differentiations of Eqs. IV-34 are

$$
d \hat{e}_{4 \alpha}=\left(r_{4 \alpha}\right)^{-2}\left(r_{4 \alpha} d \hat{r}_{4 \alpha}-\hat{r}_{4 \alpha} d r_{4 \alpha}\right)
$$

since

$$
\hat{e}_{41} \cdot \hat{e}_{42}=\cos \varphi_{3}
$$

then

$$
d \cos \varphi_{3}=-\sin \varphi_{3} d \varphi_{3}=\hat{e}_{41} \cdot d \hat{e}_{42}+\hat{e}_{42} \cdot d \hat{e}_{41}
$$

By going through the same derivations as Eqs. IV-2.I to IV-25, we get

$$
\begin{aligned}
d \varphi_{3} & =\left(r_{41} \sin \varphi_{3}\right)^{-1}\left(\cos \varphi_{3} \hat{e}_{41}-\hat{e}_{42}\right) \cdot \hat{p}_{1} \\
& +\left(r_{42} \sin \varphi_{3}\right)^{-1}\left(\cos \varphi_{3} \hat{e}_{42}-\hat{e}_{41}\right) \cdot \hat{e}_{2} \\
& +\left(r_{41} r_{42} \sin \varphi_{3}\right)^{-1}\left(\hat{r}_{41}-r_{41} \hat{e}_{42} \cos \varphi_{3}\right. \\
& \left.-r_{42} \hat{e}_{41} \cos \varphi_{3}+r_{42} \hat{e}_{42}\right) \cdot \hat{\rho}_{4}
\end{aligned}
$$

According to Eqs.'IV-33 and IV-38 we get

$$
\begin{aligned}
\hat{s}_{t \alpha}: t & =4 ; \alpha=1,2,3,4 \\
\hat{s}_{41} & =\left(r_{41} \sin \varphi_{3}\right)^{-1}\left(\cos \varphi_{3} \hat{e}_{41}-\hat{e}_{42}\right) \\
\hat{s}_{42} & =\left(r_{42} \sin \varphi_{3}\right)^{-1}\left(\cos \varphi_{3} \hat{e}_{42}-\hat{e}_{41}\right) \\
\hat{s}_{43} & =0 \\
\hat{s}_{44} & =\left(r_{41} r_{42} \sin \varphi_{3}\right)^{-1}\left[\left(r_{41}-r_{42} \cos \varphi_{3}\right) \hat{e}_{41}\right. \\
& \left.+\left(r_{42}-r_{41} \cos \varphi_{3}\right) \hat{e}_{42}\right]
\end{aligned}
$$


In formulating $t=5$ and $t=6$ the processes are exactly the same as $t=4$. Since the indexes are permutative, we can wrlte down

$$
\begin{aligned}
\hat{s}_{t \alpha}: \quad t=5 ; \alpha=1,2,3,4 & \hat{s}_{51}=0 \\
\hat{s}_{52}= & \left(r_{42} \sin \varphi_{1}\right)^{-1}\left(\cos \varphi_{1} \hat{e}_{42}-\hat{e}_{43}\right) \\
\hat{s}_{53}= & \left(r_{43} \sin \varphi_{1}\right)^{-1}\left(\cos \varphi_{1} \hat{e}_{43}-\hat{e}_{42}\right) \\
\hat{s}_{54}= & \left(r_{42} r_{43} \sin \varphi_{1}\right)^{-1}\left[\left(r_{42}-r_{43} \cos \varphi_{1}\right) \hat{e}_{42}\right. \\
& \left.\left(r_{43}-r_{42} \cos \varphi_{1}\right) \hat{e}_{43}\right] \\
\hat{s}_{t \alpha}: t= & 6 ; \alpha=1,2,3,4 \\
\hat{s}_{61}= & \left(r_{41} \sin \varphi_{2}\right)^{-1}\left(\cos \varphi_{2} \hat{e}_{41}-\hat{e}_{43}\right) \\
\hat{s}_{62}= & 0 \quad \hat{s}_{63}=\left(r_{43} \sin \varphi_{2}\right)^{-1}\left(\cos \varphi_{2} \hat{e}_{43}-\hat{e}_{42}\right) \\
\hat{s}_{64}= & \left(r_{41} r_{43} \sin \varphi_{2}\right)^{-1}\left[\left(r_{41}-r_{43}^{\cos } \varphi_{2}\right) \hat{e}_{41}\right. \\
& \left.\left(r_{43}-r_{41} \cos \varphi_{2}\right) \hat{e}_{43}\right]
\end{aligned}
$$

The Elements of "G" Matrix for Four-Center Fragment

According to Eqs. III -97 , the "G" matrix elements

in this case are

$$
g_{t t^{\prime}}=\sum_{\alpha=1}^{4} \mu_{\alpha} \hat{s}_{t \alpha} \hat{s}_{t^{\prime} \alpha}
$$

where $\mu_{\alpha}=\left(m_{\alpha}\right)^{-1} ; t, t^{\prime}=1, \ldots, 6$.

$$
G=\left(\begin{array}{ccc}
g_{11} & \cdots & g_{16} \\
\vdots & & \vdots \\
\dot{g}_{61} & \cdots & g_{66}
\end{array}\right)=\left(\begin{array}{c}
\mu_{1} \\
\mu_{2} \\
\mu_{3} \\
\mu_{4}
\end{array}\right) \cdot\left(\begin{array}{ccc}
\hat{S}_{11} & \hat{S}_{14} \\
\vdots & \vdots \\
\vdots & \vdots \\
\hat{S}_{61} & \cdots & \hat{S}_{64}
\end{array}\right) \cdot\left(\begin{array}{ccc}
\hat{S}_{11} \cdots \cdots & \hat{S}_{61} \\
\vdots & & \vdots \\
\hat{S}_{14} & \cdots & \hat{\hat{S}}_{64}
\end{array}\right)
$$


91

By substituting Eq. IV -30, IV -3I, IV -32, IV -39, IV -40 and IV -4I into Eq. IV -43 , we get the elements of the "G" matrix for the four-center fragment as follows: (IV-44)

$$
\begin{aligned}
& g_{11}=\mu_{1}+\mu_{4} \\
& g_{12}=g_{21}=\mu_{4} \cos \varphi_{3} \\
& g_{13}=g_{31}=\mu_{4} \cos \varphi_{2} \\
& g_{14}=g_{41}=\mu_{4}\left(r_{41} r_{42} \sin \varphi_{3}\right)^{-1}\left[\left(r_{42} \cos \varphi_{3}-r_{41}\right)\right. \\
& \left.+\left(r_{41} \cos \varphi_{3}-r_{42}\right) \cos \varphi_{3}\right] \\
& g_{15}=g_{51}=\mu_{4}\left(r_{42} r_{43} \sin \varphi_{1}\right)^{-1}\left[\left(r_{43} \cos \varphi_{1}-r_{42}\right) \cos \varphi_{3}\right. \\
& \left.+\left(r_{42} \cos \varphi_{1}-r_{43}\right) \cos \varphi_{2}\right] \\
& g_{16}=g_{61}=\mu_{4}\left(r_{41} r_{43} \sin \varphi_{2}\right)^{-1}\left[\left(r_{43} \cos \varphi_{2}-r_{41}\right)\right. \\
& \left.+\left(r_{41} \cos \varphi_{2}-r_{43}\right) \cos \varphi_{2}\right] \\
& g_{22}=\mu_{2}+\mu_{4} \\
& g_{23}=g_{32}=\mu_{4} \cos \varphi_{1} \\
& g_{24}=g_{42}=\mu_{4}\left(r_{41} r_{42} \sin \varphi_{3}\right)^{-1}\left[\left(r_{42} \cos \varphi_{3}-r_{41}\right) \cos \varphi_{3}\right. \\
& \left.+\left(r_{41} \cos \varphi_{3}-r_{42}\right)\right] \\
& g_{25}=g_{52}=\mu_{4}\left(r_{42} r_{43} \sin \varphi_{1}\right)^{-1}\left[\left(r_{43} \cos \varphi_{1}-r_{42}\right)\right. \\
& \left.+\left(r_{42} \cos \varphi_{1}-r_{43}\right) \cos \varphi_{1}\right] \\
& g_{26}=g_{62}=\mu_{4}\left(r_{41} r_{43} \sin \varphi_{2}\right)^{-1}\left[\left(r_{43} \cos \varphi_{2}-r_{41}\right) \cos \varphi_{3}\right. \\
& \left.+\left(r_{41} \cos \varphi_{2}-r_{43}\right) \cos \varphi_{1}\right] \\
& g_{33}=\mu_{3}+\mu_{4} \\
& g_{34}=g_{43}=\mu_{4}\left(r_{4} r_{42} r^{\ln \varphi_{3}}\right)^{-1}\left[\left(r_{42} \cos \varphi_{3}-r_{41}\right) \cos \varphi_{2}\right. \\
& \left.+\left(r_{41} \cos \varphi_{3}-r_{42}\right) \cos \varphi_{1}\right] \\
& g_{35}=g_{53}=\mu_{4}\left(r_{42} r_{43} \sin \varphi_{1}\right)^{-1}\left[\left(r_{43} \cos \varphi_{1}-r_{42}\right) \cos \varphi_{1}\right. \\
& \left.+\left(r_{42} \cos \varphi_{1}-r_{43}\right)\right] \\
& g_{36}=g_{63}=\mu_{4}\left(r_{41} r_{43} \sin \varphi_{2}\right)^{-1}\left[\left(r_{43} \cos \varphi_{2}-r_{41}\right) \cos \varphi_{2}\right. \\
& \left.+\left(r_{41} \cos \varphi_{2}-r_{43}\right)\right]
\end{aligned}
$$




$$
\begin{aligned}
& g_{44}=\mu_{1}\left(r_{41}\right)^{-2}+\mu_{2}\left(r_{42}\right)^{-2}+\mu_{4}\left(r_{41} r_{42} \text { sin } \varphi_{3}\right)^{-2}\left[\left(r_{41}-\right.\right. \\
& \left.r_{42} \cos \varphi_{3}\right)^{2}-2\left(r_{41}-r_{42} \cos \varphi_{3}\right) \\
& \left.x\left(\dot{r}_{42}-r_{41} \cos \varphi_{3}\right) \cos 3+\left(r_{42}-r_{41} \cos \varphi_{3}\right)^{2}\right] \\
& g_{45}=g_{54}=\mu_{2}\left(r_{42}^{2} \sin \varphi_{1} \sin \varphi_{3}\right)^{-1}\left(\cos \varphi_{2}-\cos \varphi_{1} \cos \varphi_{3}\right) \\
& +\mu_{4}\left(r_{41} r_{42}^{2} r_{43} \sin \varphi_{1} \sin \varphi_{3}\right)^{-1}\left\{\left(r_{41}-r_{42} \cos \varphi_{3}\right)\right. \\
& x\left[\left(r_{42}-r_{43} \cos \varphi_{1}\right) \cos \varphi_{3}\right. \\
& \left.+\left(r_{43}-r_{42} \cos \varphi_{1}\right) \cos \varphi_{2}\right] \\
& +\left(r_{42}-r_{41} \cos \varphi_{3}\right)\left[\left(r_{42}-r_{43} \cos \varphi_{1}\right)\right. \\
& \left.\left.+\left(r_{43}-r_{42} \cos \varphi_{1}\right) \cos \varphi_{1}\right]\right\} \\
& g_{46}=g_{64}=\mu_{1}\left(r_{41}^{2} \sin \varphi_{2} \sin \varphi_{3}\right)^{-1}\left(\cos \varphi_{1}-\cos \varphi_{2} * \cos \varphi_{3}\right) \\
& +\mu_{4}\left(r_{41}^{2} r_{42} r_{43} \sin \varphi_{2} \sin \varphi_{3}\right)^{-1}\left\{\left(r_{41}-r_{42} \cos \varphi_{3}\right)\right. \\
& {\left[\left(r_{41}-r_{43} \cos \varphi_{2}\right)+\left(r_{43}-r_{4.1} \cos \varphi_{2}\right) \cos \varphi_{2}\right]} \\
& +\left(r_{42}-r_{41} \cos \varphi_{3}\right)\left[\left(r_{41}-r_{43} \cos \varphi_{2}\right) \cos \varphi_{3}\right. \\
& \left.+\left(r_{43}-r_{41} \cos \varphi_{2}\right) \cos \varphi_{1}\right] \\
& g_{55}=\mu_{2}\left(r_{42}\right)^{-2}+\mu_{3}\left(r_{43}\right)^{-2}+\mu_{4}\left(r_{42} r_{43} \sin \varphi_{1}\right)^{-2}\left[\left(r_{42}-\right.\right. \\
& \left.r_{43} \cos \varphi_{1}\right)^{2}+2\left(r_{42}-r_{43} \cos \varphi_{1}\right) \\
& \left.x\left(r_{43}-r_{42} \cos \varphi_{1}\right) \cos \varphi_{1}+\left(r_{43}-r_{42} \cos \varphi_{1}\right)^{2}\right] \\
& g_{56}=g_{65}=\mu_{3}\left(r_{43}^{2} \sin \varphi_{1} \sin \varphi_{2}^{\prime}\right)^{-1}\left(\cos \varphi_{3}-\cos \varphi_{1} \cos \varphi_{2}\right) \\
& +\mu_{4}\left(r_{41} r_{42} r_{43}^{2} \sin \varphi_{1} \text { sin } \varphi_{2}\right)^{-1} \\
& x\left\{\left(r_{42}-r_{43} \cos \varphi_{1}\right)\left[r_{41}-r_{43} \cos \varphi_{2}\right) \cos \varphi_{3}\right. \\
& \left.+\left(r_{43}-r_{41} \cos \varphi_{2}\right) \cos \varphi_{1}\right]+\left(r_{43}-r_{42} \cos \varphi_{1}\right) \\
& x\left[\left(r_{41}-r_{43} \cos \varphi_{2}\right) \cos \varphi_{2}\right) \cos \varphi_{2} \\
& \left.\left.+\left(r_{43}-r_{41} \cos \varphi_{2}\right)\right]\right\} \\
& \left.g_{66}=\mu_{1}\left(r_{41}\right)^{-2}+\mu_{3}\left(r_{43}\right)^{-2}+\mu_{4}\left(r_{4}\right)_{43} r \ln \varphi_{2}\right)^{-2}\left[\left(r_{41}-\right.\right. \\
& \left.r_{43} \cos \varphi_{2}\right)^{2}+2\left(r_{41}-r_{43} \cos \varphi_{2}\right)\left(r_{43}-r_{41} \cos 2\right) \cos \varphi_{2} \\
& \left.+\left(r_{43}-r_{41} \cos \varphi_{2}\right)^{2}\right]
\end{aligned}
$$


The above derivations are the general formulations of non-linear three-center (Eqs. IV-29), planar four-center (Eqs. IV-44) cases. There are infinite many models one can set up. But they are not of interest in this investigation. The numerlcal assignments of Eqs. IV-29 and IV-44 are given in the following sections. 
3. Calculations and Resulto

Input parameters for several speciflc models were selected by arbitrarily assigning a bond order to each bond and then empirically relating bond orders to bond lengths, bond angles, and force constants.

Assigned bond orders were: for the four-center fragment, 1 for bond $2-4,2-x$ for bond $1-4$, and $1+x$ for bond 3-4; for the three-center fragment, $1+x$ for bond $2-3$ and 2 for bond $1-3$. A $\mid l$ bonds are speclfied in terms of the notation of Fig. IV-I.C. A value of the parameter $X$ from 0 to 1 was specified for each set of calculations; $x=0$ corresponds to the reactant structure and $x=1$ to the product structure, assuming enol formation in the rate-determining step.

The bond angle in the three-center fragment, $\varphi$, was assumed to be $120^{\circ}$ for $x=0,180^{\circ}$ for $x=1$, and a linear function of $x$ for intermediate values. The four-center fragment was assumed to be planar, with all three bond angles equal to $120^{\circ}$, for all values of $x$.

Bond lengths for bond orders of 1 and 2 were estimated from complled date 35 for stable molecules:

$1.54 \AA$ for $C-C, 1.33 \AA$ for $C=C, 1.43 \AA$ for $C-0$, and $1.22 \AA$ for $C=0$. These bond lengths were assumed to vary IInearly with bond, orders between 1 and 2 . In addition, smali varlations were made in the lengths of bond 2-4 in the four-center fragment and bond $1-3$ in the three-center 
fragment. As $X$ went from 0 to. 1 , the former went from $1.37 \AA$ to $1.43 \AA$ and the latter went from $1.22 \AA$ to, $1.18 \AA$.

Simllarly, stretching force constants were estimated for bond orders of 1 and 2 and were varled I inearly with intermediate bond orders. The assigned stretching force constants 29 , In millidynes/A, were 5.0 for $C-C, 9.0$ for $c=c, 6.0$ for $C-0$, and 13.0 for $c=0$. In addition, the $C=0$ stretching force constant for bond $2-3$ in the three-center fragment was varied from 13.0 to 15.0 moynes $/ \AA$ as $X$ varled from 0 to 1 .

In assigning bending force constants, the assumptions were that for the four-center fragment all $f_{\varphi \varphi} r_{j} r_{j}$ $=0.6 \mathrm{mdyne} / \AA$ and that for the three-center fragment $f_{\varphi \varphi} / r_{i} r_{j}$ varied from 0.6 to 1.0 mydne/A, Inearly with $x$. All off-diagonal force constants were set equal to zero.

The above method for specifying fragment structures and force constants is arbitrary and empirical, and it is not intended to be accurate in detail. However, it does permit a systematic variation in transition-state models from reactant to product configurations in terms of a single parameter, $x$.

The actual computations have been carried out on the PSC IBM 1620. The input data for the calculations were bond lengths, bond angles, force constants, and atomic masses. A sequence of programs was written to calculate 
the elements of $G$, the $F G$ product, the elgenvalues of the secular equations, and finally the isotope effects. (Appendix)

Three fragment models are shown in Tables, IV-l to IV-4. The fragment structures in Model 1 approximate to corresponding portions of the reactant malonlc acld. In Model 2 they approximate the product structures, except that $\varphi$ in the three-center fragment is $170^{\circ}$ rather than $180^{\circ}$. Model 3 is an intermediate case, $w /$ th $x=1 / 2$. The calculated isotope effects for these models are compared with experiment ${ }^{12}$ in Table IV-5. It can be seen that the intermediate set of fragment structures, Model 3 , gives agreement with the experimental $k_{4} / k_{3}$. For this reaction, then, a molecular-fragment calculation for a chemically reasonable model, is consistent with experiment. 
97

Table IV-I Fragment Models

4-Center
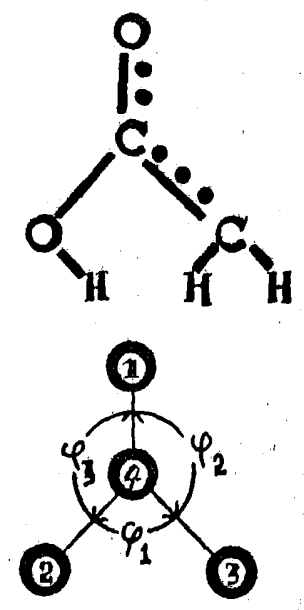

$$
\begin{aligned}
& q_{1}=\delta r_{14} \\
& q_{2}=\delta r_{24} \\
& q_{3}=\delta r_{34} \\
& q_{4}=\delta \varphi_{142} \\
& q_{5}=\delta \varphi_{243} \\
& q_{6}=\delta \varphi_{143} \\
& m_{1}=m_{2}=16 \\
& m_{3}=12 \\
& m_{4}=12, \\
& m_{4}^{1}=13
\end{aligned}
$$

3-Center

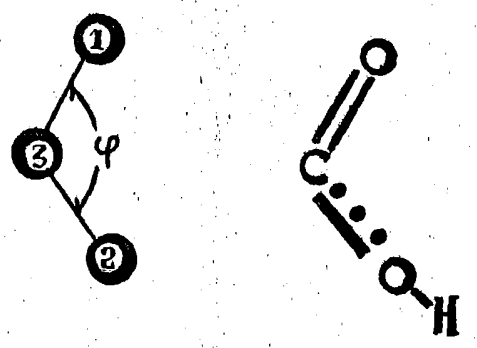

$$
\begin{aligned}
& q_{1}=\delta r_{13} \\
& q_{2}=\delta r_{23} \\
& q_{3}=\delta \varphi_{132}
\end{aligned}
$$$$
m_{1}=m_{2}=16
$$$$
m_{3}=13 \text {, }
$$$$
m_{3}^{\prime}=12
$$ 
Table IV-2 Model I - Reactant

4-Center

$r_{41}=1.22 \AA$

$r_{42}=1.37 \AA$

$r_{43}=1.54 \mathrm{~A}$

$\varphi_{142}=\varphi_{243}=\varphi_{143}=120^{\circ}$
3-Center

$$
\begin{aligned}
& r_{31}=1.22 \AA \\
& r_{32}=1.37 \AA \\
& \varphi_{132}=120^{\circ} \\
& f_{11}=13.0000 \\
& f_{22}=6.0000 \\
& f_{33}=1.0030
\end{aligned}
$$

$f_{11}=13.0000$

$f_{22}=6.0000$

$f_{33}=5.0000$

$f_{44}=1.0030$

$f_{55}=1.2660$

$f_{66}=1.1270$ 
Table IV -3 Model 2 - Product

4-Center

$$
\begin{aligned}
& r_{41}=r_{42}=1.43 \AA \\
& r_{43}=1.35 \AA
\end{aligned}
$$

$\varphi_{142}=\varphi_{243}=\varphi_{143}=120^{\circ}$

$$
\begin{aligned}
& f_{11}=6.0000 \\
& f_{22}=6.0000 \\
& f_{33}=9.0000 \\
& f_{44}=1.2270 \\
& f_{55}=1.1580 \\
& f_{66}=1.1580
\end{aligned}
$$

3 -Center

$$
\begin{aligned}
& r_{31}=1.10^{\circ} \\
& r_{32}=1.25 \mathrm{~A} \\
& \varphi=170^{\circ}
\end{aligned}
$$

$$
\begin{aligned}
& f_{11}=15.0000 \\
& f_{22}=14.0000 \\
& f_{33}=1.4750
\end{aligned}
$$


100

Table IV-4 Model 3 - Intermediate

4-Center

$$
\begin{aligned}
& r_{41}=1.33 \AA \\
& r_{42}=1.40 \AA \\
& r_{43}=1.43 \AA
\end{aligned}
$$

$$
\varphi_{142}=\varphi_{243}=\varphi_{143}=120^{\circ}
$$

$\therefore 1$.

$$
f_{11}=10.0000
$$$$
f_{22}=6.0000
$$

$f_{33}=7.0000$

$f_{44}=1.1170$

$f_{55}=1.2010$

$f_{66}=1.1410$
3-Center

$$
\begin{aligned}
& r_{31}=1.20 \AA \\
& r_{32}=1.30 \AA
\end{aligned}
$$

$$
\begin{aligned}
& \varphi=1.50^{\circ} \\
& f_{11}=14.0000 \\
& f_{22}=10.0000 \\
& f_{33}=1.2480
\end{aligned}
$$


Table IV-5 Calculated and Experimental Values of $k_{4} / k_{3}$

$$
k_{4} / k_{3} \text { at }
$$

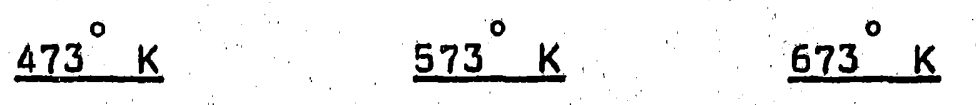

$\begin{array}{llll}\text { Model } 1 & 1.041 & 1.031 & 1.024 \\ \text { Model } 2 & 1.010 & 1.006 & 1.004 \\ \text { Model } 3 & 1.026 & 1.019 & 1.015 \\ \text { Experiment } & 1.023 \pm 0.004 & 1.017 \pm 0.004 & 1.013 \pm 0.004\end{array}$




\section{Chapter V - Summary and Further Calculations}

1. General Surnmary

103

2. Further Calculations

106 
1. General Summary

By assuming the validity of quasi-equilibrium-theory $(Q E T)$, the kinetic rate constant for a unimolecular reaction can be formulated. By the method of isotope substitution one can determine the kinetic rate-constant ratlo $\mathrm{k}^{1} / \mathrm{k}$, which provides rather detalled and valuable information of the transition state.

For two reactions with identical reactants, the rate constant ratio depends only upon the respective transition states. Assuming two identical geometries in the transition state with difference only in isotopic constitution, the ratio of rate constants for unimolecular reactions can be written as

$$
\frac{k^{\prime}}{k}=\frac{\nu_{x}^{\prime}}{\nu_{x}} \prod_{i}^{3 n-6} \frac{u_{1} \sinh \left(\frac{U_{i}}{2}\right)}{U_{i}^{\prime} \sinh \left(\frac{U_{1}}{2}\right)} \quad \prod_{i}^{3 n-7} \quad \frac{U_{1}^{\neq 0} \sinh \left(\frac{U_{1}^{*}}{2}\right)}{U_{1}^{\neq} \sinh \left(\frac{U_{1}^{\neq}}{2}\right)}
$$

where primed and unprimed quantities refer to the two Isotoplc species and Frefers to the transition-state structure, $\nu_{x}$ is the frequency of motion in the reaction coordinate, and $U_{p}=h c w_{q} / K T$ where $\omega_{p}$ is a vibrational frequency. The calculation of vibrational frequencles for each structure requires solution of a matrix equation of order $3 n-7$ or $3 n-6$ where $n$ is the number of atoms, and becomes extremely complicated for molecules of moderate size. However, In bond-rupture processes, In spite of 
the usual sense of transition-s tate theory, it was assumed that the bond-rupture is already completed in the transition 8 tate and that motion in the reaction coordinate can be treated as simple translational separation of molecular fragments. Then the calculations are simplifled a great deal. Furthermore, by applying stern and Wolfsberg's "cut-off" approximation, the hydrogen atoms (non-isotoplc sites) are omitted.

The specific problem treated in this investigation is carbon isotope effect in the decarboxylation of malonic acld.

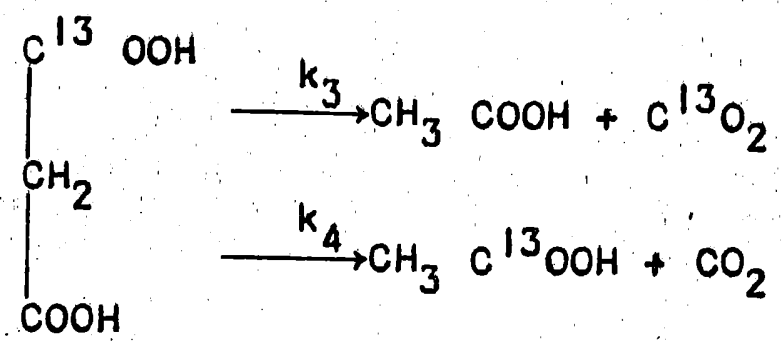

Malonic acid is approximated as a seven-center model

an?

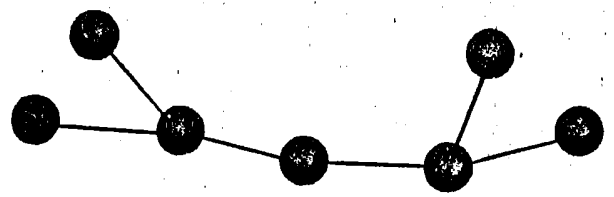

and the transition state is approximated by three- and four-center fragments.

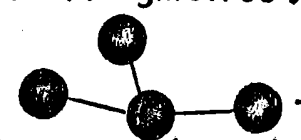

Thus, we have reduced a 26 th order secular equation to one 6 th order and one 3 rd order secular equation. The general method of calculation is to set up the 
105

$F$ and $G$ matrices according to Wilson's method.

$$
|F G-E \lambda|=0 \text { where } \lambda_{1}=4 \pi^{2} c^{a} \omega_{1}^{2}
$$

The elf genvalue and eigenvector iteration and matrix-deflation methods are employed to solve our equations. A sequence of computer programs has been written, and the calculations have been carried out on the PSC IBM 1620. The calculated results are in good agreement with the presently available experimental data.

The applicability of this method can be extended to reactions involving simultaneous and step-wise multiple bond-rupture and formation. It can also be used to estimate substituent effects in a series of analogous isotopic reactions. 
2. Further Calculations

From the previous results (Table IV -5 ), we see that Model 3 provided agreement with the experimental data. since the cholce of force constants was arbitrary and empirical as descrlbed in chapter IV, it would be essential to test the sensitivity of the isotope effects with respect to the change in force constants.

since Model 3 in Chapter IV is a chemically reasonable model, two varlations of Model 3 have been chosen to be calculated. The geometrical conflgurations are chosen to be the same as Model 3 in Table IV-4. The model descriptions and the results are listed in Table $V-1$ to $V-3$.

The model described in Table $V-1$ is denoted as Model $3 \mathrm{~A}$ where the force constants have been increased by ten percent of the original force constants used in Model 3 calculations. The model described in table $V-2$, denoted as Model 3B, Is exactiy the same as Model 3 except that the force constants have been decreased by ten per cent.

It can be seen from Table $V-3$ that for the decarboxylation of malonic acid the molecular-fragment calculations for a range of chemically reasonable models are consistent with experiment. However, this agreement does not necessarily confirm the success of thls method of calculation. In order to test the general valldity of this method, even for this speciflc reaction, a 
107

comparison with the result of more detailed calculation is desirable. Theoretically, this simplified approach should provide as good a result as any other rigorous method. Then a complete mathematical analysis of this type of problem should be of general interest. 
Table V-1 Model 3A - Intermediate structure With $10 \%$ Increase in the Force Constants

\section{4-Center}

$$
\begin{aligned}
& r_{41}=1.33 \AA \\
& r_{42}=1.40 \AA \\
& r_{43}=1.43 \AA \\
& \varphi_{142}=\varphi_{243}=\varphi_{143}=120^{\circ} \\
& f_{11}=11.0000 \\
& f_{22}=6.6000 \\
& f_{33}=7.7000 \\
& f_{44}=1.2287 \\
& f_{55}=1.3211 \\
& f_{66}=1.2551
\end{aligned}
$$

\section{3-Center}

$$
\begin{aligned}
& r_{31}=1.20^{\circ} \AA \\
& r_{32}=1.30^{\circ} \\
& \varphi=150^{\circ}
\end{aligned}
$$$$
f_{11}=15,4000
$$$$
f_{22}=11.0000
$$$$
f_{33}=1.3728
$$ 
109

Table V-2 Model 3B - Intermediate structure

With $10 \%$ Decrease in the Force Constants

4-Center

$$
\begin{aligned}
& r_{41}=1.33 \AA \\
& r_{42}=1.40 \AA \\
& r_{43}=1.43 \AA
\end{aligned}
$$

$$
\varphi_{142}=\varphi_{243}=\varphi_{143}=120^{\circ}
$$

$$
\begin{aligned}
& f_{11}=9.0000 \\
& f_{22}=5.4000 \\
& f_{33}=6.3000 \\
& f_{44}=1.0053 \\
& f_{55}=1.0009 \\
& f_{66}=1.0269
\end{aligned}
$$

3-Center

$$
\begin{aligned}
& r_{31}=1.20 \AA \\
& r_{32}=1.30 \AA \\
& \varphi=150^{\circ}
\end{aligned}
$$

$$
\begin{aligned}
& f_{11}=12.6000 \\
& f_{22}=9.0000 \\
& f_{33}=1.1232
\end{aligned}
$$


110

Table V-3 Calculated and Experimental Values of $k_{4} / k_{3}$

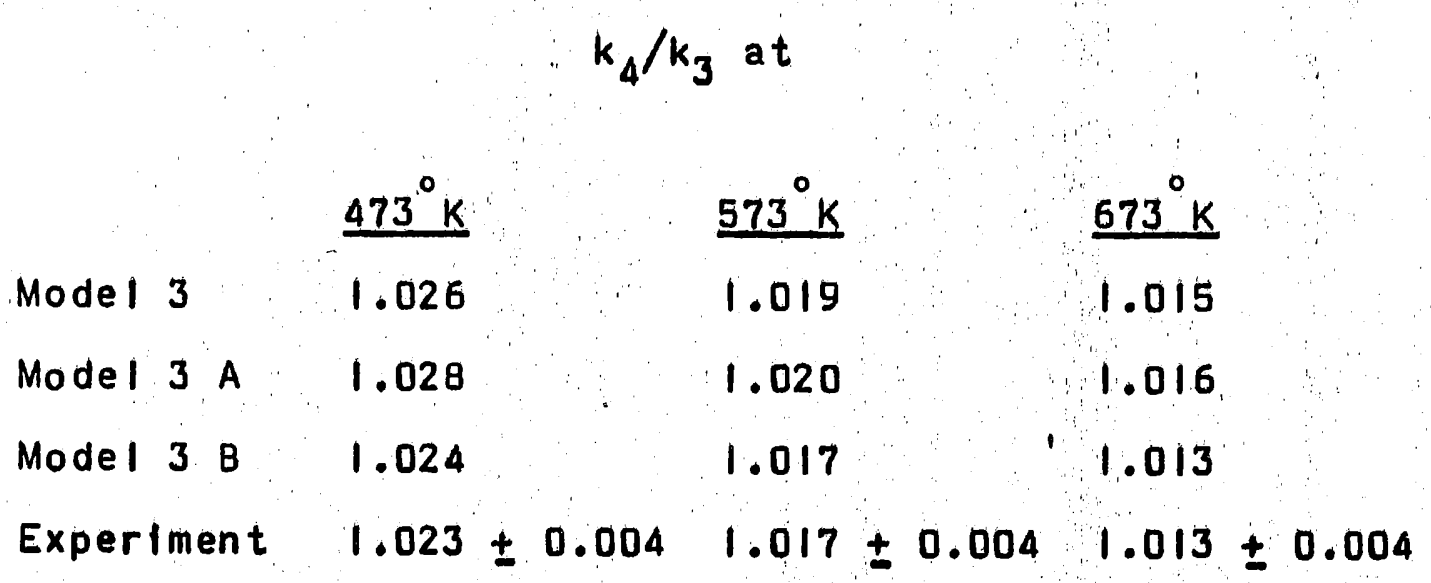


APPENDIX - COMPUTER PROGRAMS

1. Main Program

2. "G" Matrix
112

114

A. Three-Center Fragment

B. Four-Center Fragment $A$

C. Four-Center Fragment $B$

3. Elgen-Value Problem

123

4. Isotopic KInetic Rate Ratio

127 
1. Main Program

\section{ZZJOB 5}

ZZFOR 5

*POBJP4

DIMENSION $A(6,6), B(6,6), C(6,6), X \operatorname{L1}(6), X \operatorname{L} 2(6), X \operatorname{LL}(6)$, $X L 4(6)$

COMMON A, B,C,XL1,XL2,XL3,XL4,U1,U2,U3,U4, RADI, RAD2, RAD3

COMMON COMPI,COMP2,COMP3,R4I,RA2,R43

I CALL THREE

$\operatorname{CALL} \operatorname{EIGEN}(C, X L 1,3)$

CALL THREE

$\operatorname{CALL} \operatorname{EIGEN}(C, X L 2,3)$

CALL SIXA

CALL SIXB

$\operatorname{CALL} \operatorname{EIGEN}(C, X L 3,6)$

CALL SIXA

CALL SIXB

CALL EIGEN $(C, X L 4,6)$.

CALL RATIO

GO TO 1

2. $Q A=A(1,1)$

$Q A=\operatorname{SQRTF}(Q A)$

$Q A=A B S F(Q A)$

$Q A=\cos F(1.0)$

$Q A=\operatorname{SIN} F(1.0)$ 
113

3

FORMAT(EI6.8)

PRINT 3, ( (C(I,J), I=1,3), J=1,3)

$Q A=Q A * * 2$

$Q A=\operatorname{EXP} F(1.0)$

$Q A=\operatorname{LOGF}(Q A)$

END

ZZZZ 
2. "G" MATRIX

A. THREE-CENTER MODEL

ZZJOB 5

ZZFOR 5

* LOISKTHREE

SUBROUTINE THREE

C

C DEFINE G MATRIX AND THE PRODUCT OF $G$ AND $F$

DIMENSI ON $A(6,6), B(6,6), C(6,6), X \operatorname{LI}(6), X \operatorname{LL}(6), X \operatorname{CL}(6)$,

$X L 4(6)$

COMMON A, B, C,XL1, XL2, XL3, XL4,U1,U2,U3,U4,RADI, RAD2,

RAD3

COMMON COMPI, COMP2, COMP3,R41,R42,R43

1 FORMAT(F8.5,2F5,3,3F8.6, II)

2 FORMAT( $9 F 6.4$ )

3 FORMAT $(6(F 10.5,2 x))$

99 FORMAT $(/(3 F 10.5,2 x))$

199 FORMAT $(/ / 3 F 10.5,2 X))$

10 READ 1, ANGR3 I, R3 2, AM1, AM2, AM3

PUNCH $3, A N G, R 31, R 32, A M 1, A M 2, A M 3$

$U I=1.0 / \mathrm{AMI}$

$U 2=1.0 / \mathrm{AM} 2$

$U 3=1.0 / \mathrm{AM} 3$

$R A D=A N G * 3.141592 / 180.0$

C

C DEFINE THE ELEMENTS OF G(A) MATRIX 
C

C

$$
\begin{aligned}
& A(1,1)=U 1+U 3 \\
& A(2,1)=U 3 * C O 3 \quad F(R A D) \\
& A(3,1)=-U 3 * S I N F(R A D) / R 32 \\
& A(1,2)=A(2,1) \\
& A(2,2)=U 2+U 3 \\
& A(3,2)=-U 3 * S I N F(R A D) / R 31 \\
& A(1,3)=A(3,1) \\
& A(2,3)=A(3,2) \\
& A(3,3)=U 1 / R 31 * 2+U 2 / R 32 * 2+U 3 *(1.0 / R 31 * 2+ \\
& \mid .0 / R 32 * 22-12.0 * \operatorname{COS} F(R A D) /(R 31 * R 32))
\end{aligned}
$$

C

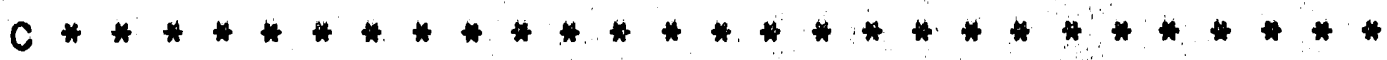

C

DO $7 \mathrm{~K}=1,3$

7 PUNCH 99, $(A(K, I), I=1,3)$

READ 2, $((B(I, J), I-1,3), J=1,3)$

DO $B \quad J=1,3$

B PUNCH 99, $(B(I, J), I=1,3)$

TSUM $=0.0$

DO $20 \mathrm{~K}=1,3$

DO $6 \mathrm{~J}=1,3$

DO $5 \quad I=1,3$

$\operatorname{SUM}=A(K, I) * B(I, J)$

5 TSUM $=$ TSUM+SUM

$$
c(K, J)=\text { TSUM }
$$


6 TSUM $=0.0$

20. PUNCH 199, $(C(K, J), J=1,3)$

RETURN

END

ZZZZ 
B. Six-Center Model (A)

ZZJOB 5

ZZFOR 5

*LKISKSIXA

SUBROUTINE SIXA

DIMENSION $A(6,6), B(6,6), C(6,6), X \operatorname{CL}(6), X \operatorname{XL}(6), X \operatorname{LL}(6)$, $\mathrm{XLA}(6)$

COMMON A, $B, C, X L 1, X L 2, X L 3, X L 4, U 1, U 2, U 3, U 4, R A D 1, R A D 2$, RAD3

COMMON COMPI,COMP2,COMP3, R4 I,R42,R43

I FORMAT(IO(FG.2))

3 FORMA T $(/ / 5(F / 0.4))$

READ 1,ANG1, ANG2, ANG3, R41, R42, R43, AM1, AM2, AM3, AM4

PUNCH 3, ANG1, ANG2, ANG3, R41, R42,R43, AMI, AM2, AM3, AM4

$U 1=1.0 / A M I$

$U 2=1.0 / A_{2}$

$U 3=1.0 / \mathrm{AM} 3$

$U_{4}=1.0 /$ AM4

RADI $=A N G 1 * 3.141592 / 180.0$

$R A D 2=A N G 2 * 3.141592 / 180.0$

$R A D 3=A N G * 3.141592 / 180.0$

COMPI $=\cos F(R A D 3) *(R 41-R 42 * \cos F(R A D 3)) *(R 42-R 43 *$ $\cos F(R A D I))+1 \cos F(R A D 2) *(R 41-R 42 * \cos F(R A D 3)) *(R 43-$ $R 42 * \cos F(R A D I))+(R 42-2 R 41 * \cos F(R A D 3)) *(R 42-R 43 * \cos$ $F(R A D I))+\cos F(R A D I) *(R 42-3 R 4 I * \cos F(R A D 3)) *(R 43-$ $R 42 * \cos F(R A D I))$ 
$\operatorname{COMP2}=(R 41-R 42 * \cos F(R A D 3)) *(R 41-R 43 * \operatorname{COS} F(R A D 2))+$ $\cos F(R A D 2) * \mid(R 41-R 42 * \cos F(R A D 3)) *(R 43-R 41 * \cos$ $F(R A D 2))+\cos F(R A D 3) *(R 42-2 R 41 * \cos F(R A D 3)) *(R 41-$ $R 43 * \cos F(R A D 2))+\cos F(R A D I) *(R 42-3 R 41 * \cos F(R A D 3)) *$ $(R 43-R 41 * \operatorname{Cos} F(R A D 2))$ COMP3 $=(R 42-R 43 * \cos F(R A D I)) *(R 41-R 43 * \cos F(R A D 2)) *$ $\mid \cos F(R A D 3)+(R 42-R 43 * \cos F(R A D I)) *(R 43-R 4 \mid * \cos$ $F(R A D 2)) * 2 \cos F(R A D I)+(R 43-R 42 * \cos F(R A D I)) *(R 41-$ $R 43 * \cos F(R A D 2)) * 3 \cos F(R A D 2)+(R 43-R 42 * \cos F(R A D 1)) *$ $(R 43-R 41 * \cos F(R A D 2))$

C

C DEFINE VARIABLES OF MATRIX $G(A)$

C

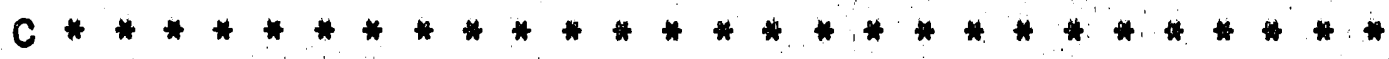
C

$$
\begin{aligned}
& A(1,1)=U 1+U 4 \\
& A(1,2)=U 4 * \cos F(R A D 3) \\
& A(1,3)=U 4 * \cos F(R A D 2) \\
& A(1,4)=U 4 *((R 42 * \cos F(R A D 3)-R 41)+\cos F(R A D 3) *
\end{aligned}
$$

$(R 41 * \cos F(R A D 3)-\mid R 42)) / R 41 * R 42 * \operatorname{SIN} F(R A D 3))$

$$
A(1,5)=U 4^{*}(\cos F(R A D 3) *(R 43 * \cos F(R A D 1)-R 42)+\cos
$$
$F(R A D 2) *(R 42 * I \operatorname{COS} F(R A D I)-R 43)) /(R 42 * R 43 * S I N F(R A D I))$ $A(1,6)=U 4 *((R 43 * \cos F(R A D 2)-R 41)+\cos F(R A D 2) *(R 4) *$ $\cos F(R A D 2)-\mid R 43)) /(R 4 \mid * R 43 * 8 I N F(R A D 2))$

$$
\begin{aligned}
& A(2,1)=A(1,2) \\
& A(2,2)=U 2+U 4 \\
& A(2,3)=U_{4} * \operatorname{Cos} F(\text { RAD })
\end{aligned}
$$


$A(2,4)=U 4 *((R 41 * \cos F(R A D 3)-R 42)+\cos F(R A D 3) *(R 42 *$ $\cos F(R A D 3)-\mid R 41)) /(R 4 \mid * R 42 * \sin F(R A D 3))$

$$
A(2,5)=U_{4} *((R 43 * \cos F(R A D I)-R 42)+\cos F(R A D I) *
$$

$(R 42 * C 08 F(R A D I)-1 R 43)) /(R A 2 * R 43 * 81 N F(R A O I))$

$$
A(2,6)=U 4 *(\operatorname{Cos} F(R A D 3) *(R 43 * \cos F(R A D 2)-R 41)+
$$

$1 \cos F(R A D 1) *(R 41 * \cos F(R A D 2)-R 43)) /(R 41 * R 43 * S I N$ $F(R A D 2))$

$$
\begin{aligned}
& A(3,1)=A(1,3) \\
& A(3,2)=A(2,3) \\
& A(3,3)=U 3+U 4 \\
& A(3,4)=U 4 *(\operatorname{Cos} F(R A D 2) *(R 42 * \cos F(R A D 3)-R 41)+
\end{aligned}
$$
$1 \cos F(R A D I) *(R 41 * \cos F(R A D 3)-R 42)) /(R 41 * R 42 * 8 I N$ $F(\operatorname{RAD} 3))$

$$
\begin{aligned}
& A(3,5)=U 4 *((R 42 * \cos F(R A D I)-R 43)+\cos F(R A D I) * \\
& (R 43 * \cos F(R A D I)-\mid R 42)) /(R 42 * R 43 * \operatorname{SIN} F(R A D I)) \\
& A(3,6)=U 4 *((R 4 \mid * \cos F(R A D 2)-R 43)+\cos F(R A D 2) * \\
& (R 43 * \cos F(R A D 2)-\mid R 41)) /(R 41 * R 43 * \operatorname{SIN} F(R A D 2)) \\
& \text { RETURN } \\
& \text { END }
\end{aligned}
$$


C. Six-Center Model' (B)

ZZJOB 5

ZZFOR 5

*LDI SKSI XB

SUBROUTINE SIXB

DIMENSION $A(6,6), B(6,6), C(6,6), X \operatorname{L2}(6), X \operatorname{L} 3(6)$,

$X L 4(6)$

COMMON A , B, C, XL1, XL2, XL3, XL4,U1, U2, U3, U4, RADI, RAD2, RAD3

COMMON COMPI, COMP2,COMP3,R4I,R42,R43

C DEFINE VARIABLES OF MATRIX $G(A)$

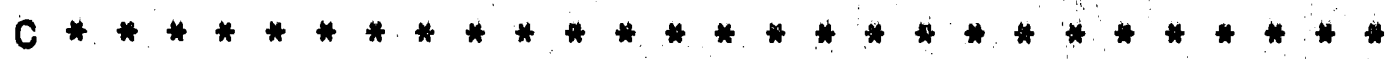

$$
\begin{aligned}
& A(4,1)=A(1,4) \\
& A(4,2)=A(2,4) \\
& A(4,3)=A(3,4) \\
& A(4,4)=U 1 / R 41 * * 2+U 2 / R 42 * 2+U 4 *((R 4)-R 42 * \cos
\end{aligned}
$$

$F(R A D 3)) * * 2-12.0 * \cos F(R A D 3) *(R 41-R 42 * \cos F(R A D 3)) *$

$(R 42-R 41 * \cos F(R A D 3))+2(R 42-R 41 * \cos F(R A D 3)) * * 2) /(41 *$ R42*SIN F(RAD3)**2

$$
A(4,5)=U 2 *(\cos F(R A D 2)-\cos F(R A D 1) * \cos F(R A D 3)) /
$$

$(R 42 * * 2 * I S I N$ F $(R A D I) * \operatorname{SIN~} F(R A D 3))+U 4 * \operatorname{COMPI/}$

$2(R 41 *(R 42 * * 2) * R 43 * \operatorname{SIN} F(R A D I) * \operatorname{SIN} F(R A D 3))$

$$
A(4,6)=U 1 *(\cos F(R A D I)-\cos F(R A D 2) * \cos F(R A D Z)) /
$$

$I((R 41 * * 2) * \operatorname{SIN} F($ RAD2)*SIN $F(R A D 3)) * U 4 * \operatorname{COMP} 2 /$

$((R 41 * * 2) * R 42 * R 43 * 2 S I N F(R A D 2) * S I N F(R A D 3))$ 


$$
\begin{aligned}
& A(5,1)=A(1,5) \\
& A(5,2)=A(2,5) \\
& A(5,3)=A(3,5) \\
& A(5,4)=A(4,5) \\
& A(5,5)=U 2 / R 42 * 2+U 3 / R 43 * 2+U 4 *((R 42-R 43 * \operatorname{Cos}
\end{aligned}
$$

$F(R A D I)) * * 2+12.0 *(R 42-R 43 * \cos F(R A D I)) *(R 43-R 42 * \cos$

$F(R A D I)) * \cos F(R A D I)+2(R 43-R 42 * \cos F(R A D I)) * * 2) /$

$(R 42 * R 43 * 8 I N F(R A D I)) * * 2$

$$
A(5,6)=U 3 *(\cos F(R A D 3)-\cos F(R A D 1) * \cos F(R A D 2)) /
$$

(R43**2*ISIN $F(R A D I) * S I N ~ F(R A D 2))+U 4 * C O M P 3 /(R 41 * R 42 *$

$R 43 * 2 * 2 S I N F(R A D I) * S I N F(R A D 2))$

$$
\begin{aligned}
& A(6,1)=A(1,6) \\
& A(6,2)=A(2,6) \\
& A(6,3)=A(3,6) \\
& A(6,4)=A(4,6) \\
& A(6,5)=A(5,6) \\
& A(6,6)=U 1 / R 41 * 2+U 3 / R 43 * * 2+U 4 *((R 4 \mid-R 43 * \cos \\
& F(R A D 2)) * 2+\mid 2.0 * \cos F(R A D 2) *(R 4 \mid=R 43 * \cos \\
& F(R A D 2)) *(R 43-R 41 * \cos F(R A D 2))+2(R 43-R 41 * \cos \\
& F(R A D 2)) * * 2) /(R 41 * R 43 * \operatorname{SIN} F(R A D 2)) * 2
\end{aligned}
$$

C

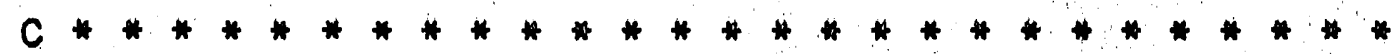

C

2 FORMAT(F6.4,F6.4,F6.4,F6.4,F6.4,F6.4)

$9 \operatorname{FORMAT}(/ / 6(F \theta .4,2 x))$

99. FORMAT $(/ / 6(F 9.6,2 X))$

102 FORMAT $(/ / 6(F 10.6,2 x))$ 
DO $7 \mathrm{~K}=1,6$

7 PUNCH 102, $(A(K, I), I=1,6)$

DO $10 \mathrm{~L}=1,6$

10 READ $2,(B(I, L), I=1,6)$

DO $8 \mathrm{~K}=1,6$

8 PUNCH 9, $(B(J, K), J=1,6)$

TSUM $=0.0$

DO $20 \mathrm{~K}=1,6$

DO $6 \mathrm{~J}=1,6$

DO $5 \mathrm{I}=1,6$

SUM $=A(K, I) * B(I, J)$

5 TSUM $=$ TSUM+SUM

$C(K, J)=T S U M$

6 TSUM $=0.0$

20 PUNCH 99, $(C(K, J), J=1,6)$

100 RETURN

END 
3. Elgen-Value Problem

ZZJOB 5

ZZFOR 5

* LDISKEIGEN O170

SUBROUTINE EIGEN $(A, X L, N)$

DIMENSION $X(6,2), X L(6)$

DIMENSION $A(6,6), B(6,6), C(6,6), X \operatorname{XL}(6), X L 2(6)$,

$X \operatorname{L3}(6), X \operatorname{LA}(6)$

COMMON COMPI,COMP2,COMP3,R4I,R42,R43

$K=N$

$K R=1$

19. $K A=0$

$\mathrm{KN}=30$

C INITILIZE TRIAL EIGENVECTOR

$X L(K R)=0.0$

DO $1 \mathrm{I}=1, \mathrm{~K}$

$1 \quad x(1,1)=1.0$

C MULTIPLY BY MATRIX

9 DO $2 \mathrm{I}=1, \mathrm{~K}$

$x(1,2)=0.0$

DO $2 \mathrm{~J}=1, \mathrm{~K}$

$2 \quad x(1,2)=A(1, J) * x(J, 1)+X(1,2)$

C FIND LARGEST ELEMENT

$$
\begin{aligned}
& L=I \\
& R=A B S F(X(1,2)) \\
& D O 3 I=2, K \\
& R I=A B S F(X(I, 2))
\end{aligned}
$$


I $F(R=R I) 4,3,3$

$4 \quad L=1$

$R=R \mid$

3 CONTINUE

C STORE APPROXIMATION TO LAMBDA

$$
\begin{aligned}
& R I=A B S F(X L(K R)-X(L, 2))-A B S F(X L(K R)) \\
& I F(R I) 41,41,42
\end{aligned}
$$

$42 \quad K N=K N-1$

$\operatorname{IF}(K N) 43,43,41$

43 PRINT 44

44 FORMAT( I7HLAMBDA IS COMPLEX)

DO $55 I=1, K$

55 PUNCH 46, $(A(I, J), J=l, K)$

46 FORMAT(//2HCM,3E20.8/10X,3E20.8)

PAUSE

IF(SENSE SWI TCH 1)47,48

48 CALL EXIT

47 DO 49 I $=K R, N$

PRINT 50

50 FORMAT(34HENTER ONE VALUE IN F TYPE NOTATION)

ACCEPT $51, \times L(1)$

51 FORMAT(F20.4)

49 CONTINUE

GO TO 30

$41 \quad X L(K R)=X(L, 2)$

C TEST FOR LAMBDA APP. $=0.0$

IF $(X L(K R)) 5,6,5$ 
$6 \operatorname{IF}(K A) B, 7,8$

$7 \quad K A=1$

$x(1,1)=x(1,1)-1.0$

GO TO 9

C TEST FOR COMPLETION OF CURRENT I TERATION

5

$$
A I=0.0
$$

DO $10 \quad I=1, K$

$X(I, 2)=X(1,2) / X L(K R)$

$A I=A I+A B S F(x(I, 1)=x(1,2)$

$10 \quad x(I, 1)=x(I, 2)$

IF $(A \mid-.00001) \mid 1,11,9$

C START MATRIX DEFLATION

II $J=L+1$

$$
\text { IF }(L-K) 110,12,12
$$

110 DO $13 \quad I=J, K$

$$
x(1-1,2)=x(1,2)
$$

DO 13 IA $=1, K$

$13 \quad A(I A, I-1)=A(I A, I)$

$12 \quad K I=K-1$

DO $14 \quad I=1, K 1$

14. $X(I, I)=A(L, I)$

$$
\text { IF }(L-K) 15,16,16
$$

$15 \quad 00 \quad 17 \quad I=J, K$

$$
\text { DO } 17 \quad\{A=1, K
$$

$17 \quad A(I-1, I A)=A(I, I A)$

C COMPLETE MATRIX DEFLATION

16 DO $18 \quad I=1, \mathrm{~K} 1$ 


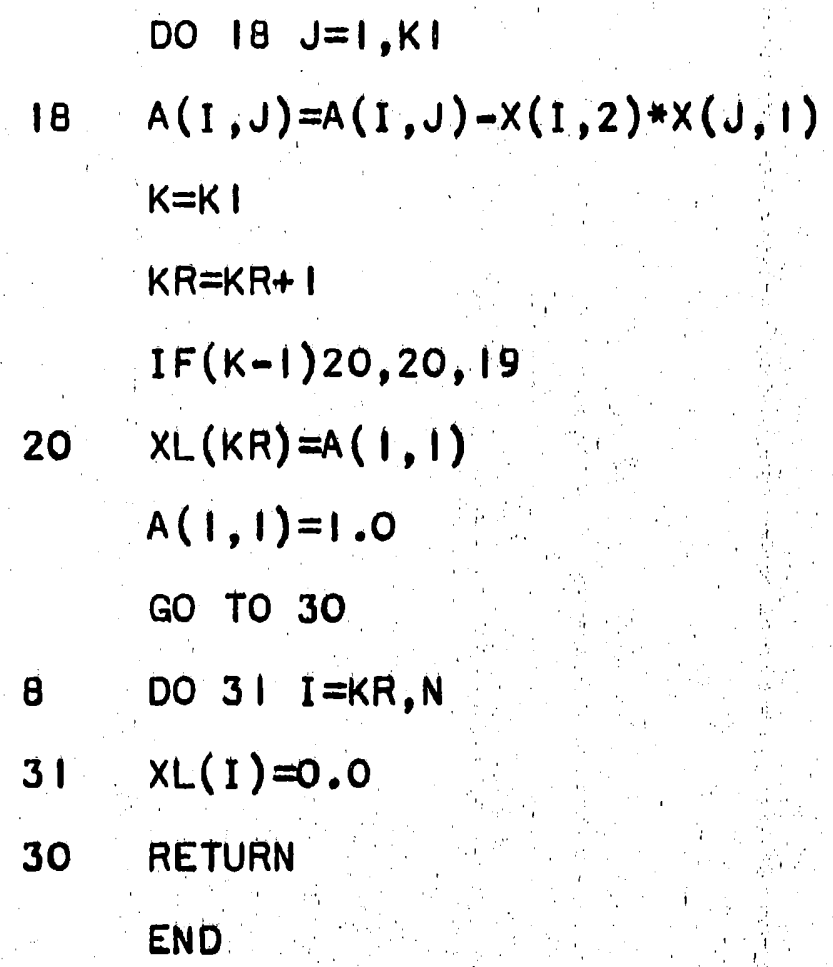


4. ISOTOPIC KINETIC RATE RATIO

ZZJOB 5

ZZFOR 5

* LoI SKRA tIo

SUBROUTINE RATIO

C

c * * * * OBTAIN the IsOTOP kINETIC RATIO*******

c

DIMENSION $F(6), F P(6), W(6), W P(6)$

DIMENSION $A(6,6), B(6,6), C(6,6), X \operatorname{LI}(6), X \operatorname{LL}(6)$,

$X L 3(6), X L 4(6)$

COMMON A , B, C, XL1,XL2,XL3,XL4,U1,U2,U3,U4,RADI,RAD2。 RAD3

COMMON COMPI, COMP2, COMP3,R4I,R42,R43

1 FORMAT(F6.4,F6.4,FG.4,F6.4)

2 FORMAT(F10.6)

3 FORMAT( $/(E \mid 6.08))$

4 FORMAT(GF |0.6)

C

C

READ MASSES FOR BOTH MODEL'S

C

I $S=0$

50 READI, AM1, AM2, AM3

$A M=A M 1+A M 2+A M 3$

READI , AMI , AM2, AM3 , AM4

$F M=A M 1+A M 2+A M 3+A M 4$

I F(I ) $51,51,52$ 
$51 U=A M * B M /(A M+B M)$

IS $=1$

GO TO 50

$52 U P=A M * B M /(A M+B M)$

Is $=0$

C

C

C

$R=U / U P$

$R=\operatorname{SQRTF}(R)$

PUNCH $3, R$

READ2, T

C

C

CALCULATE WAVELENGTH CONBTANT

C

$F A C=(.1177 E+05) * 7.0 /(T * 44.0)$

PUNCH3, FAC

C

C

ENTER CONTINUOUS MULTIPLICATION LOOP

C

$N=3$

DO $54 \mathrm{~K}=1, \mathrm{~N}$

$W(K)=X L I(K)$

PUNCH 3,W(K)

$W P(K)=X L 2(K)$

54 PUNCH 3 ,WP(K)

53 DO $100 \quad L L=1, N$ 


$$
\begin{aligned}
& F(L L)=F A C * \operatorname{SQRTF}(W(L L)) \\
& F P(L L)=F A C * \operatorname{SQRTF}(W P(L L))
\end{aligned}
$$

$A I=F(L L) / 2.0$

$A 2=F P(L L) / 2.0$

$R=R^{*}(\operatorname{SQRTF}(W P(L L)) / \operatorname{SQRTF}(W(L L))) * \operatorname{EXPF}(A \mid-A 2)$

$101 R=R *(1.0-\operatorname{EXPF}(-F(L L))) /(1.0-\operatorname{EXPF}(-F P(L L)))$

PUNCH 3,R

100 CONTINUE

IF(I8) $110,110,150$

110 is $=1$

$N=6$

DO $55 \mathrm{~K}=1, \mathrm{~N}$

$W(K)=X L 3(K)$

PUNCH $3, W(K)$

$W P(K)=X L 4(K)$

55 PUNCH 3 ,WP(K)

GO TO 53

150 PUNCH3, R

RETURN

END 


\section{BIBLIOGRAPHY}

1. W. Heisenberg

$$
\text { Zelts. f. Physik, 43, } 172 \text { (1927). }
$$

N. Bohr

Nature, $121,500(1928)$.

2. F. A. Lindemann

Phil. Mag., 37, 523 (1919); 38, 173 (1920)。

3. H. C. Urey and D. Rittenberg

J. Chem. Phys., 1, 137 (1933).

4. H. C. Urey

J. Chem. Soc., 562 (1947).

5. J. W. Blgeleisen and M. G. Mayer

J. Chem. Phys., 15, 261 (1947).

6. O. Beeck, J.W. Otvos, P. D. Stevenson, and C. D. Wagner J. Chem. Phys., 16, 255 (1948).

7. J. Bigeleisen

J. Chem. Phys., 17, 675 (1949).

B. J. C. Polanyl

J. Chem. Phys., 23, 1505 (1955).

9. R. E. Weston

J. Chem. Phys., 31, 892 (1959).

10. I. Shavitt

J. Chem. Phys., 31, 1359 (1959).

11. P. E. Yankwich and M. Calvin

J. Chem. Phys., 17, 109 (1949).

12. C. R Gatz

Ph.D. Thesis, U. of Illinols (1960). 
13. L. Winelmy

$$
\text { Pogg. Ann., 81, 413, } 499 \text { (1850). }
$$

14. C. M. Guldberg and P. Waage

"Études sur les Affinltés Chimiques",

Brogger and Christie, Christianla (0s|O) (1867).

15. S. Arrhenlus

Z. Physik Chem., 4, 226 (1889).

16. M. Born and J.R. Oppenheimer Ann. Physik 84, 457 (1927).

17. R. H. Fowler and E. A. Guggenhelim

"Statistical Thermodynamics", Cambrldge U. Press New York, (1939).

18. D. M. Bishop and K. J. Laidler

J. Chem. Phys., 42, 1688 (1965).

K. J. Laldler and J. C. Polanyl

"Progress in Reaction Kinetics" Vol. III

Pergamon Press, oxford, (1965).

19. H. Eyring

"Reaction Kinetics - the 67 th General Discusstion

of the Faraday Society" Gurney and Jackson,

London (1937).

20. E. P. Wigner

Z. Physik Chem., B 19, 903 (1932).

C. Eckart

Phys, Rev., 35, 1303 (1930). 
R. P. Bell

Proc. Roy. Soc. (London), A 139,446 (1933).

Trans. Faraday Soc., 55, I (1959).

21. N. B. Slater

Proc. Roy, Soc. (London), A 194, 112 (1948).

22. J.W. Bigeleisen and $M$. Wolfsberg

"Advances in Chemfcal Physics" Vol. 1. Interscience

Publishers Inc., New York (1958).

23. J.W. Blgelelsen and M. Wolfsberg

J. Chem. Phys., 21, $1972(1953), 22,1264$ (1954)

24. C. R. Gatz

J. Chem. Phys., 44, 1861 (1966).

25. R. Karplus

J. Chem. Phys., 16, 1170 (1948).

H. H. Nielsen

Revs. Mod. Phys., 23, 90 (1951).

26. C. Eckart

Phys. Rev., 47, 552 (1935).

27. M. Ellashevich

compt, rend, acad, sci, U.R.S.S., 28:605 (1940).

28. E. B. Wilson, Jr.

J. Chem. Phys., 7:1047 (1939).

29. E. B. Wlison, Jr., J. C. Declus, and P. C. Cross

"Molecular Vibrations" McGraw-Hill Book Co., Inc., New York, N. Y. (1955).

30. H. S. Johnston, W. A. Bonner, and D.J.Wilson

J. Chem. Phys., 26, 1002 (1957). 
133

31. M. Wolfsberg and M. J. Stern

Pure App. Chem., 8, 225 (1964).

32. B. S. Roblnovitch and D. W. Seiser

Adv. Photochem., 3, I (1964).

33. M. Knudsen

"The Kinetic Theory of Gases", Methuen and Co., Ltd., London (1952).

34. M. J. Stern and M. Wolfsberg

J. Chem. Phys., 45, 4105 (1966).

35. L. E. Sutton, ed.

"Tables of Interatomic Distances and Configurations In Molecules and Ions", Special Publication \#II, The Chemical Society, London, 1958. 